\title{
CATEGORICAL ACTIONS ON UNIPOTENT REPRESENTATIONS OF FINITE CLASSICAL GROUPS
}

\author{
O. DUDAS, M. VARAGNOLO, E. VASSEROT
}

\begin{abstract}
We review the categorical representation of a Kac-Moody algebra on unipotent representations of finite unitary groups in non-defining characteristic given in [18], using Harish-Chandra induction and restriction. Then, we extend this construction to finite reductive groups of types $\mathrm{B}$ or $\mathrm{C}$ in non-defining characteristic. We show that the decategorified representation is isomorphic to a direct sum of level 2 Fock spaces. We deduce that the Harish-Chandra branching graph coincides with the crystal graph of these Fock spaces. We also obtain derived equivalences between blocks, yielding Broué's abelian defect group conjecture for unipotent $\ell$ blocks at linear primes $\ell$.
\end{abstract}

\section{Contents}

Introduction

1. Categorical representations

1.1. Rings and categories

1.2. Kac-Moody algebras of type $A$ and their representations

1.2.1. Lie algebra associated with a quiver

1.2.2. Integrable representations

1.2.3. Quantized enveloping algebras

1.3. Categorical representations on abelian categories

1.3.1. Affine Hecke algebras and representation data

1.3.2. Categorical representations

1.4. Minimal categorical representations

1.5. Crystals

1.6. Perfect bases

1.7. Derived equivalences

2. Representations on Fock spaces

2.1. Combinatorics of $l$-partitions

2.1.1. Partitions and $l$-partitions

2.1.2. Residues and content

2.1.3. $\quad l$-cores and $l$-quotients

This research was partially supported by the ANR grant number ANR-13-BS01-0001-01. 
2.2. Fock spaces

2.3. Charged Fock spaces

2.3.1. The $\mathfrak{g}$-action on the Fock space

2.3.2. The crystal of the Fock space

3. Unipotent representations

19

3.1. Basics

3.2. Unipotent $K G$-modules

3.3. Unipotent $\mathrm{k} G$-modules and $\ell$-blocks

3.4. Harish-Chandra series 23

4. Finite unitary groups 24

4.1. Definition 25

4.2. The representation datum on $R G$-mod 26

4.3. The categories of unipotent modules $\mathscr{U}_{K}$ and $\mathscr{U}_{\mathrm{k}}$

4.3.1. The category $\mathscr{U}_{K} \quad 27$

4.3.2. The category $\mathscr{U}_{\mathrm{k}}$

4.3.3. Blocks of $\mathscr{U}_{\mathrm{k}} \quad 29$

4.3.4. The weak Harish-Chandra series 29

4.4. The $\mathfrak{g}_{\infty}$-representation on $\mathscr{U}_{K}$

4.4.1. Action of $E$ and $F$

4.4.2. The ramified Hecke algebra 31

4.4.3. Parametrization of the weak Harish-Chandra series of $\mathscr{U}_{K}$

4.4.4. The $\mathfrak{g}_{\infty}$-representation on $\mathscr{U}_{K}$

4.5. The $\mathfrak{g}_{e}$-representation on $\mathscr{U}_{\mathrm{k}}$. 33

4.5.1. The Lie algebras $\mathfrak{g}_{e}$ and $\mathfrak{g}_{e, \circ}$

4.5.2. The $\mathfrak{g}_{e}^{\prime}$-action on $\left[\mathscr{U}_{\mathrm{k}}\right] \quad 34$

4.5.3. The $\mathfrak{g}_{e}$-action on $\left[\mathscr{U}_{\mathrm{k}}\right]$

4.5.4. The $\mathfrak{g}_{e}$-action on $\mathscr{U}_{\mathrm{k}}$

4.6. Derived equivalences of blocks of $\mathscr{U}_{\mathrm{k}} \quad 35$

4.6.1. Characterization of the blocks of $\mathscr{U}_{\mathrm{k}} \quad 36$

4.6.2. Derived equivalences of blocks of $\mathscr{U}_{\mathrm{k}} \quad 36$

4.7. The crystals of $\mathscr{U}_{K}$ and $\mathscr{U}_{\mathrm{k}}$

4.7.1. Crystals and Harish-Chandra series 36

4.7.2. Comparison of the crystals 37

5. The representation of the Heisenberg algebra on $\mathscr{U}_{\mathrm{k}} \quad 38$

5.1. The Heisenberg action on $\left[\mathscr{U}_{\mathrm{k}}\right] \quad 38$

5.1.1. The Heisenberg algebra $\quad 38$

5.1.2. The Heisenberg action on $\mathbf{F}(Q) \quad 38$

5.1.3. The Heisenberg action on $\left[\mathscr{U}_{\mathrm{k}}\right] \quad 39$

5.2. The modular Harish-Chandra series of $G L_{m} \quad 40$

5.2.1. The unipotent modules of $G L_{n}$ 
5.2.2. The modular Steinberg character and Harish-Chandra series 41

5.3. The Heisenberg functors 41

5.4. The categorification of the Heisenberg action on $\left[\mathscr{U}_{\mathrm{k}}\right] \quad 42$

5.5. Cuspidal modules and highest weight vectors 46

5.5.1. The parameters of the ramified Hecke algebras 47

5.5.2. The theorem 47

5.5.3. Cuspidal modules and FLOTW e-partitions 49

6. Types B and C 49

6.1. Definitions 50

6.1.1. Odd-dimensional orthogonal groups 50

6.1.2. Symplectic groups 50

6.2. The representation datum on $R G$-mod 51

6.3. The categories of unipotent modules $\mathscr{U}_{K}$ and $\mathscr{U}_{\mathrm{k}} \quad 52$

6.3.1. Parametrization by symbols $\quad 53$

6.3.2. The unipotent modules over $K$

6.3.3. The unipotent modules over $\mathrm{k} \quad 5.5$

6.3.4. The unipotent blocks 55

6.4. The $\mathfrak{g}_{\infty}$-representation on $\mathscr{U}_{K} \quad 56$

6.4.1. The ramified Hecke algebra 56

6.4.2. The $\mathfrak{g}_{\infty}$-representation on $\mathscr{U}_{K} \quad 60$

6.5. The $\mathfrak{g}_{2 d}$-representation on $\mathscr{U}_{\mathrm{k}}$

6.5.1. The $\mathfrak{g}_{2 d^{\prime}}^{\prime}$-representation on $\mathscr{U}_{\mathrm{k}} \quad 61$

6.5.2. The $\mathfrak{g}_{2 d}$-representation on $\mathscr{U}_{\mathrm{k}}$ in the linear prime case 63

6.5.3. Combinatorics of $d$-cohooks and $d$-cocores 64

6.5.4. The weight of a symbol 66

6.5.5. The $\mathfrak{g}_{2 d^{-}}$-representation on $\mathscr{U}_{\mathrm{k}}$ in the unitary case 68

6.5.6. Determination of the ramified Hecke algebras 70

6.6. Derived equivalences $\quad 71$

6.7. The crystal of $\mathscr{U}_{\mathrm{k}} \quad 71$

6.7.1. Ordering symbols $\quad 72$

6.7.2. Parametrization of unipotent modules $\quad 72$

6.7.3. Comparison of the crystals 73

References 74

\section{INTRODUCTION}

Let $\mathbf{G}$ be a connected algebraic group defined over a finite field $\mathbb{F}_{q}$. The finite group $G=\mathbf{G}\left(\mathbb{F}_{q}\right)$ of its $\mathbb{F}_{q}$-rational points is a finite reductive group. The irreducible representations of $G$ over fields of characteristic $\ell$ prime to $q$ fall into Harish-Chandra 
series, which are defined in terms of Harish-Chandra induction $R_{L}^{G}$ and restriction ${ }^{*} R_{L}^{G}$ from proper Levi subgroups $L$ of $G$. The isomorphism classes in each series are parametrized by the simple modules of the ramified Hecke algebras, which are realized as the endomorphism algebras of the Harish-Chandra induction of cuspidal representations. Therefore, the classification of isomorphism classes of irreducible representations of $G$ can be reduced to the following two problems :

(a) classification of the cuspidal irreducible representations,

(b) determination of the ramified Hecke algebras.

This was achieved by Lusztig in [54] when $\ell=0$ but it remains open for representations in positive characteristic for most of the finite reductive groups. By results of Geck-Hiss-Malle [32, we know however that the ramified Hecke algebras are indeed Hecke algebras of finite type, only the parameters of the deformation are unknown in general.

When $G$ is a classical group, it turns out that most of the structure of the ramified Hecke algebras does not depend on the corresponding unipotent cuspidal representations. This suggests to rather study the endomorphism algebra of the HarishChandra induction functor $R_{L}^{G}$ rather than the endomorphism algebra of the induced representation. This was already achieved in [10] for $G=\mathrm{GL}_{n}(q)$. Our goal is to extend Chuang-Rouquier's approach to other classical groups.

In the first part of this paper we focus on the case of finite unitary groups $\operatorname{GU}_{n}(q)$, reviewing our previous work [18], whereas in the last part we deal with the case of groups of types B or C. We will work with both ordinary representations (characteristic zero) and modular representations in non-defining characteristic (characteristic $\ell$ prime to $q$ ). More precisely, the field of coefficients $R$ of the representations will be an extension of either $\mathbb{Q}_{\ell}$ or $\mathbb{F}_{\ell}$.

Let $\mathrm{G}_{n}(q)$ be one of the families of finite classical groups among $\mathrm{GU}_{2 n}(q), \mathrm{GU}_{2 n+1}(q)$, $\mathrm{Sp}_{2 n}(q)$ and $\mathrm{SO}_{2 n+1}(q)$. Using the tower of inclusion of groups $\cdots \subset \mathrm{G}_{n}(q) \subset$ $\mathrm{G}_{n+1}(q) \subset \cdots$ one can form the abelian category of unipotent representations

$$
\mathscr{U}_{R}:=\bigoplus_{n \geqslant 0} \mathrm{G}_{n}(q) \text {-umod }
$$

Furthermore, under mild assumption on $\ell$, we can modify the Harish-Chandra induction and restriction functors to obtain an adjoint pair $(E, F)$ of functors on $\mathscr{U}_{R}$. The functor $F$ corresponds to a Harish-Chandra induction from $\mathrm{G}_{n}(q)$ to $\mathrm{G}_{n+1}(q)$ whereas $E$ corresponds to the restriction. Note that only specific Levi subgroups are considered, and we must work with a variation of the usual Harish-Chandra theory (the weak Harish-Chandra theory) introduced in [36].

In this framework, problem (a) amounts to finding the modules $V$ such that $E V=$ 0 and problem (b) is about the structure of $\operatorname{End}_{G}\left(F^{m} V\right)$ for such cuspidal modules $V$. 
As mentioned before, most of the structure of this endomorphism algebra is already contained in $\operatorname{End}\left(F^{m}\right)$. In $\$ 4.2$ and $\$ 6.2$, we construct natural transformations $X$ of $F$ and $T$ of $F^{2}$ which endow $\operatorname{End}\left(F^{m}\right)$ with a morphism from an affine Hecke algebra $\mathbf{H}_{m}^{q^{\delta}}$ of type $A_{m-1}^{(1)}$ with parameter $q^{\delta}$ where the integer $\delta$ is determined as follows

- $\delta=2$ if $\mathrm{G}_{n}(q)$ is $\mathrm{GU}_{2 n}(q)$ or $\mathrm{GU}_{2 n+1}(q)$,

- $\delta=1$ if $\mathrm{G}_{n}(q)$ is $\operatorname{Sp}_{2 n}(q)$ or $\mathrm{SO}_{2 n+1}(q)$.

Back to our original problem, the evaluation at a cuspidal module $V$ provides a natural map $\mathbf{H}_{m}^{q^{\delta}} \rightarrow \operatorname{End}_{G}\left(F^{m} V\right)$. Then, we prove that this map induces a natural isomorphism between $\operatorname{End}_{G}\left(F^{m} V\right)$ and a level 2 cyclotomic quotient of $\mathbf{H}_{m}^{q^{\delta}}$ whose parameters are naturally attached to $V$, see Theorems 4.8 and 6.5.

Next, we prove that the eigenvalues of $X$ belong to

- $I_{2}=(-q)^{\mathbb{Z}}$ if $\mathrm{G}_{n}(q)$ is $\mathrm{GU}_{2 n}(q)$ or $\mathrm{GU}_{2 n+1}(q)$,

- $I_{1}=q^{\mathbb{Z}} \sqcup\left(-q^{\mathbb{Z}}\right)$ if $\mathrm{G}_{n}(q)$ is $\operatorname{Sp}_{2 n}(q)$ or $\mathrm{SO}_{2 n+1}(q)$.

Then, we can form a Lie algebra $\mathfrak{g}$ corresponding to the quiver with vertices $I_{\delta}$ and arrows given by multiplication by $q^{\delta}$. When working in characteristic zero, the Lie algebra $\mathfrak{g}$ is isomorphic to two copies of $\mathfrak{s l}_{\mathbb{Z}}$. In positive characteristic $\ell$ prime to $q$ it will depend on whether $-q$ is a power of $q^{\delta}$. This reflects the difference of behaviour for unipotent representations when working at linear or unitary primes. To explain this we denote by $d, e, f$ the order of $q^{2},-q$ and $q$ modulo $\ell$. Two situations occur:

- $\ell$ is a linear prime if $\mathrm{G}_{n}(q)$ is $\mathrm{GU}_{2 n}(q)$ or $\mathrm{GU}_{2 n+1}(q)$ and $e$ is even (then $e=2 d$ and $-q \notin q^{2 \mathbb{Z}} \bmod \ell$ ), or if $\mathrm{G}_{n}(q)$ is $\operatorname{Sp}_{2 n}(q)$ or $\mathrm{SO}_{2 n+1}(q)$ and $f$ is odd (then $f=d$ and $-q \notin q^{\mathbb{Z}} \bmod \ell$ ). In that case $\mathfrak{g}$ is a subalgebra of $\left(\widehat{\mathfrak{s l}}_{d}\right)^{\oplus 2}$;

- $\ell$ is a unitary prime if $\mathrm{G}_{n}(q)$ is $\mathrm{GU}_{2 n}(q)$ or $\mathrm{GU}_{2 n+1}(q)$ and $e$ is odd (then $e=d$ and $\left.-q \equiv\left(q^{2}\right)^{(e+1) / 2} \bmod \ell\right)$, or if $\mathrm{G}_{n}(q)$ is $\operatorname{Sp}_{2 n}(q)$ or $\mathrm{SO}_{2 n+1}(q)$ and $f$ is even (then $f=2 d$ and $-q \equiv q^{d+1} \bmod \ell$ ). In that case $\mathfrak{g}$ is isomorphic to $\widehat{\mathfrak{s l}}_{f / \delta}$.

In each case we prove that the representation datum $(E, F, X, T)$ induces a categorical action of $\mathfrak{g}$ on $\mathscr{U}_{R}$. See Theorems 4.11 and 4.17 for finite unitary groups, and Theorems 6.11, 6.12 and 6.18 for groups of types $\mathrm{B}$ and $\mathrm{C}$.

In particular, let $E=\bigoplus E_{i}$ and $F=\bigoplus F_{i}$ be the decomposition of the functors into generalized $i$-eigenspaces for $X$. Then $\left[E_{i}\right],\left[F_{i}\right]$ act as the Chevalley generators of $\mathfrak{g}$ on the Grothendieck group $\left[\mathscr{U}_{R}\right]$ of $\mathscr{U}_{R}$ and many problems on $\mathscr{U}_{R}$ have a Lietheoretic counterpart. For example,

- weakly cuspidal modules correspond to highest weight vectors,

- the decomposition of $\mathscr{U}_{R}$ into weak Harish-Chandra series corresponds to the decomposition of the $\mathfrak{g}$-module $\left[\mathscr{U}_{R}\right]$ into a direct sum of irreducible highest weight modules, 
- the parameters of the ramified Hecke algebra attached to a weakly cuspidal unipotent module $V$ are given by the weight of $[V]$,

- the blocks of $\mathscr{U}_{R}$, or equivalently the unipotent $\ell$-blocks, correspond to the weight spaces for the action of $\mathfrak{g}$ (inside a Harish-Chandra series if $\ell$ is a linear prime).

Such observations were already used in other situations, e.g., for cyclotomic rational double affine Hecke algebras.

For this dictionary to be efficient one needs to determine the $\mathfrak{g}$-module structure on $\left[\mathscr{U}_{R}\right]$. This is done in $\$ 4.5$ and $\$ 6.5$ by looking at the action of $\left[E_{i}\right]$ and $\left[F_{i}\right]$ on the basis of $\left[\mathscr{U}_{R}\right]$ formed by unipotent characters and their $\ell$-reduction, which play a role similar to the role of the standard modules in the categorifications of cyclotomic rational double affine Hecke algebras mentioned above. On this basis the action can be made explicit, and we prove that there is a natural $\mathfrak{g}$-module isomorphism

$$
\left[\mathscr{U}_{R}\right] \stackrel{\sim}{\longrightarrow} \bigoplus_{t \geqslant 0} \mathbf{F}\left(Q_{t}\right)
$$

between the Grothendieck group of $\mathscr{U}_{R}$ and a direct sum of level 2 Fock spaces $\mathbf{F}\left(Q_{t}\right)$, each of which corresponds to an ordinary Harish-Chandra series. Through this isomorphism, the basis of unipotent characters (or their $\ell$-reduction) is sent to the standard monomial basis.

Our original motivation for constructing a categorical action of $\mathfrak{g}$ on $\mathscr{U}_{R}$ comes from a conjecture of Gerber-Hiss-Jacon 36] for finite unitary groups, which predicts an explicit relation between the Harish-Chandra branching graph and the crystal graph of the Fock spaces $\bigoplus_{t \geqslant 0} \mathbf{F}\left(Q_{t}\right)$ when $e$ is odd. See also [37]. Using our categorical methods and the unitriangularity of the decomposition matrix we obtained in 18 a complete proof of the conjecture, see Theorem 4.21. We extend here this result to groups of type B and C (see Theorem 6.22). Note however that in order to do so, we must rely on a conjecture of Geck concerning the unitriangular shape of the decomposition matrices that we have recalled in Conjecture 6.21.

A similar result can be deduced when $\ell$ is a linear prime. However, in that case, the situation is already well-understood by the work of Gruber-Hiss 39 on classical groups. The case where $\ell$ is unitary is considered as more challenging and our categorification techniques give the first major result in that direction since the case of $\mathrm{GL}_{n}(q)$ was solved by Dipper-Du [16]. This solves completely the problem of classification of irreducible unipotent modules for unitary groups and groups of types $\mathrm{B}$ or $\mathrm{C}$ mentioned at the beginning of the introduction, and yields a combinatorial description of the weak Harish-Chandra series.

For finite unitary groups, another categorical construction can be used in order to get the usual (non weak) Harish-Chandra series, by adapting some techniques from [62. It relies on a categorification of the Heinsenberg representation of level 2 on the 
Fock spaces $\mathbf{F}\left(Q_{t}\right)$ mentioned above. We explain this in $\$ 5$. This yields a complete classification of the cuspidal unipotent modules, as explained in Theorem 5.10 and $\$ 5.5 .3$ (and a computation of the parameters of all ramified Hecke algebras).

By the work of Chuang-Rouquier, categorical actions also provide derived equivalences between weight spaces. In our situation, these weight spaces are exactly the unipotent $\ell$-blocks and we obtain many derived equivalences between blocks with the same local structure. Together with Livesey's construction of good blocks in the linear prime case, we deduce a proof of Broué's abelian defect group conjecture see Theorems 4.19 (or [18]) and 6.20 (which does not rely on Conjecture 6.21).

The paper is organized as follows. In Section 1 we set our notations and recall basic facts on categorical actions, perfect bases and derived equivalences. In Section 2 we introduce the Fock spaces, which are certain integrable representations of Kac-Moody algebras. They have a crystal graph which can be defined combinatorially. In Section 3 we recall standard results on unipotent representations of finite reductive groups in non-defining characteristic. In Section 4 we recall the categorical representation on the unipotent modules of unitary groups given in [18, and the main applications proved there. In Section 5 we explain the role of the Heisenberg categorical action and its relation with Harish-Chandra series. The results here are new and were announced in [18. Section 6] deals with the categorical representation on the unipotent modules of groups of type B,C and its applications to weak Harish-Chandra series and derived equivalences. The results in this final section are new.

\section{Categorical Representations}

Throughout this section, $R$ will denote a noetherian commutative domain (with unit).

\subsection{Rings and categories.}

An $R$-category $\mathscr{C}$ is an additive category enriched over the tensor category of $R$ modules. All the functors $F$ on $\mathscr{C}$ will be assumed to be $R$-linear. Given such a functor, we denote by $1_{F}$ or sometimes $F$ the identity element in the endomorphism ring $\operatorname{End}(F)$. The identity functor on $\mathscr{C}$ will be denoted by by $1_{\mathscr{C}}$. A composition of functors $E$ and $F$ is written as $E F$, while a composition of morphisms of functors (or natural transformations) $\psi$ and $\phi$ is written as $\psi \circ \phi$. We say that $\mathscr{C}$ is Homfinite if the Hom spaces are finitely generated over $R$. If the category $\mathscr{C}$ is abelian or exact, we denote by $[\mathscr{C}]$ the complexified Grothendieck group and by $\operatorname{Irr}(\mathscr{C})$ the set of isomorphism classes of simple objects of $\mathscr{C}$. The class of an object $M$ of $\mathscr{C}$ in the Grothendieck group is denoted by $[M]$. An exact endofunctor $F$ of $\mathscr{C}$ induces a linear map on $[\mathscr{C}]$ which we will denote by $[F]$. 
Assume that $\mathscr{C}$ is Hom-finite. Given an object $M \in \mathscr{C}$ we set $\mathscr{H}(M)=\operatorname{End}_{\mathscr{C}}(M)^{\mathrm{op}}$. It is an $R$-algebra which is finitely generated as an $R$-module. We denote by $\mathfrak{F}_{M}$ the functor $\mathfrak{F}_{M}=\operatorname{Hom}_{\mathscr{C}}(M,-): \mathscr{C} \longrightarrow \mathscr{H}(M)-\bmod$.

Assume now that $\mathscr{C}=H$-mod, where $H$ is an $R$-algebra with 1 which is finitely generated and free over $R$. We abbreviate $\operatorname{Irr}(H)=\operatorname{Irr}(\mathscr{C})$. Given an homomorphism $R \rightarrow S$, we can form the $S$-category $S \mathscr{C}=S H$-mod where $S H=S \otimes_{R} H$. Given another $R$-category $\mathscr{C}^{\prime}$ as above and an exact functor $F: \mathscr{C} \rightarrow \mathscr{C}^{\prime}$, then $F$ is represented by a projective object $P \in \mathscr{C}$. We set $S F=\operatorname{Hom}_{S \mathscr{C}}(S P,-): S \mathscr{C} \rightarrow$ $S \mathscr{C}^{\prime}$.

Let $K$ be the field of fractions of $R, A \subset R$ be a subring which is integrally closed in $K$ and $\theta: R \rightarrow \mathrm{k}$ be a ring homomorphism into a field $\mathrm{k}$ such that $\mathrm{k}$ is the field of fractions of $\theta(A)$. If $\mathrm{k} H$ is split, then there is a decomposition map $d_{\theta}:[K H-\bmod ] \longrightarrow[\mathrm{kH}-\mathrm{mod}]$, see e.g. [33, sec. 3.1] for more details.

\subsection{Kac-Moody algebras of type $A$ and their representations.}

The Lie algebras which will act on the categories we will study will always be finite sums of Kac-Moody algebras of type $A_{\infty}$ or $A_{e-1}^{(1)}$. They will arise from quivers of the same type.

1.2.1. Lie algebra associated with a quiver. Let $v \in R^{\times}$and $I \subset R^{\times}$. We assume that $v \neq 1$ and that $I$ is stable by multiplication by $v$ and $v^{-1}$ with finitely many orbits. To the pair $(I, v)$ we associate a quiver $I(v)$ (also denoted by $I$ ) as follows:

- the vertices of $I(v)$ are the elements of $I$;

- the arrows of $I(v)$ are $i \rightarrow i v$ for $i \in I$.

Since $I$ is assumed to be stable by multiplication by $v$ and $v^{-1}$, such a quiver is the disjoint union of quivers of type $A_{\infty}$ if $v$ is not a root of unity, or of cyclic quivers of type $A_{e-1}^{(1)}$ if $v$ is a primitive $e$-th root of 1 .

The quiver $I(v)$ defines a symmetric generalized Cartan matrix $A=\left(a_{i j}\right)_{i, j \in I}$ with $a_{i i}=2, a_{i j}=-1$ when $i \rightarrow j$ or $j \rightarrow i$ and $a_{i j}=0$ otherwise. To this Cartan matrix one can associate the (derived) Kac-Moody algebra $\mathfrak{g}_{I}^{\prime}$ over $\mathbb{C}$, which has Chevalley generators $e_{i}, f_{i}$ for $i \in I$, subject to the usual relations.

More generally, let $\left(\mathrm{X}_{I}, \mathrm{X}_{I}^{\vee},\langle\bullet, \bullet\rangle_{I},\left\{\alpha_{i}\right\}_{i \in I},\left\{\alpha_{i}^{\vee}\right\}_{i \in I}\right)$ be a Cartan datum associated with $A$, i.e., we assume that

- $\mathrm{X}_{I}$ and $\mathrm{X}_{I}^{\vee}$ are free abelian groups,

- the simple coroots $\left\{\alpha_{i}^{\vee}\right\}$ are linearly independent in $\mathrm{X}_{I}^{\vee}$,

- for each $i \in I$ there exists a fundamental weight $\Lambda_{i} \in \mathrm{X}_{I}$ satisfying $\left\langle\alpha_{j}^{\vee}, \Lambda_{i}\right\rangle_{I}=\delta_{i j}$ for all $j \in I$,

$\bullet\langle\bullet, \bullet\rangle_{I}: \mathrm{X}_{I}^{\vee} \times \mathrm{X}_{I} \longrightarrow \mathbb{Z}$ is a perfect pairing such that $\left\langle\alpha_{j}^{\vee}, \alpha_{i}\right\rangle_{I}=a_{i j}$. 
Let $\mathrm{Q}_{I}^{\vee}=\bigoplus \mathbb{Z} \alpha_{i}^{\vee}$ be the coroot lattice and $\mathrm{P}_{I}=\bigoplus \mathbb{Z} \Lambda_{i}$ be the weight lattice. Then, the Kac-Moody algebra $\mathfrak{g}_{I}$ corresponding to this datum is the Lie algebra generated by the Chevalley generators $e_{i}, f_{i}$ for $i \in I$ and the Cartan algebra $\mathfrak{h}=\mathbb{C} \otimes \mathrm{X}_{I}^{\vee}$. An element $h \in \mathfrak{h}$ acts by $\left[h, e_{i}\right]=\left\langle h, \alpha_{i}\right\rangle e_{i}$. The Lie algebra $\mathfrak{g}_{I}^{\prime}$ is the derived subalgebra $\left[\mathfrak{g}_{I}, \mathfrak{g}_{I}\right]$.

Example 1.1. When $I=v^{\mathbb{Z}}$ two cases arise.

(a) If $I$ is infinite, then $\mathfrak{g}_{I}^{\prime}$ is isomorphic to $\mathfrak{s l}_{\mathbb{Z}}$, the Lie algebra of traceless matrices with finitely many non-zero entries.

(b) If $v$ has finite order $e$, then $I$ is isomorphic to a cyclic quiver of type $A_{e-1}^{(1)}$. We can form $\mathrm{X}^{\vee}=\mathrm{Q}^{\vee} \oplus \mathbb{Z} \partial$ and $\mathrm{X}=\mathrm{P} \oplus \mathbb{Z} \delta$ with $\left\langle\partial, \Lambda_{i}\right\rangle=0,\left\langle\partial, \alpha_{i}\right\rangle=\delta_{i 1}$ and $\delta=\sum_{i \in I} \alpha_{i}$. The pairing is non-degenerate, and $\mathfrak{g}_{I}$ is isomorphic to the Kac-Moody algebra

$$
\widehat{\mathfrak{s l}}_{e}=\mathfrak{s l}_{e}(\mathbb{C}) \otimes \mathbb{C}\left[t, t^{-1}\right] \oplus \mathbb{C} c \oplus \mathbb{C} \partial .
$$

An explicit isomorphism sends $e_{v^{i}}$ (resp. $f_{v^{i}}$ ) to the matrix $E_{i, i+1} \otimes 1$ (resp. $\left.E_{i+1, i} \otimes 1\right)$ if $i \neq e$ and $e_{1}$ (resp. $\left.f_{1}\right)$ to $E_{e, 1} \otimes t$ (resp. $\left.E_{1, e} \otimes t^{-1}\right)$. Via this isomophism the central element $c$ corresponds to $\sum_{i \in I} \alpha_{i}^{\vee}$, and the derived algebra $\mathfrak{g}_{I}^{\prime}$ to $\widetilde{\mathfrak{s l}}_{e}=\mathfrak{s l}_{e}(\mathbb{C}) \otimes \mathbb{C}\left[t, t^{-1}\right] \oplus \mathbb{C} c$.

To avoid cumbersome notation, we may write $\mathfrak{g}=\mathfrak{g}_{I}, \mathrm{P}=\mathrm{P}_{I}, \mathrm{Q}^{\vee}=\mathrm{Q}_{I}^{\vee}$, etc. when there is no risk of confusion.

1.2.2. Integrable representations. Let $V$ be a $\mathfrak{g}$-module. Given $\omega \in \mathrm{X}$, the $\omega$-weight space of $V$ is $V_{\omega}=\left\{v \in V \mid \alpha^{\vee} \cdot v=\left\langle\alpha^{\vee}, \omega\right\rangle v, \forall \alpha^{\vee} \in \mathrm{Q}^{\vee}\right\}$. We denote by $\mathcal{O}^{\text {int }}$ the category of integrable highest weight modules, i.e. $\mathfrak{g}$-modules $V$ satisfying

- $V=\bigoplus_{\omega \in \mathrm{X}} V_{\omega}$ and $\operatorname{dim} V_{\omega}<\infty$ for all $\omega \in \mathrm{X}$,

- the action of $e_{i}$ and $f_{i}$ is locally nilpotent for all $i \in I$,

- there exists a finite set $F \subset \mathrm{X}$ such that $\operatorname{wt}(V) \subset F+\sum_{i \in I} \mathbb{Z}_{\leqslant 0} \alpha_{i}$.

Let $\mathrm{X}^{+}=\left\{\omega \in \mathrm{X} \mid\left\langle\alpha_{i}^{\vee}, \omega\right\rangle \in \mathbb{N}\right.$ for all $\left.i \in I\right\}$ be the set of integral dominant weights. Given $\Lambda \in \mathrm{X}^{+}$, we denote by $\mathbf{L}(\Lambda)$ the simple integrable highest weight module with highest weight $\Lambda$.

1.2.3. Quantized enveloping algebras. Let $u$ be a formal variable and $A=\mathbb{C}\left[u, u^{-1}\right]$. Let $U_{u}(\mathfrak{g})$ be the quantized enveloping algebra over $\mathbb{C}(u)$. Let $U_{A}(\mathfrak{g}) \subset U_{u}(\mathfrak{g})$ be Lusztig's divided power version of $U_{u}(\mathfrak{g})$. For each integral weight $\Lambda$ the module $\mathbf{L}(\Lambda)$ admits a deformed version $\mathbf{L}_{u}(\Lambda)$ over $U_{u}(\mathfrak{g})$ and an integral form $\mathbf{L}_{A}(\Lambda)$ which is the $U_{A}(\mathfrak{g})$-submodule of $\mathbf{L}_{u}(\Lambda)$ generated by the highest weight vector $|\Lambda\rangle$. Let $\mathcal{O}_{u}^{\text {int }}$ be the category consisting of the $U_{u}(\mathfrak{g})$-modules which are (possibly infinite) direct sums of $\mathbf{L}_{u}(\Lambda)$ 's. If $V_{u} \in \mathcal{O}_{u}^{\text {int }}$, then its integral form $V_{A}$ is the corresponding 
sum of the modules $\mathbf{L}_{A}(\Lambda)$. It depends on the choice of a family of highest weight vectors of the constituents of $V_{u}$.

\subsection{Categorical representations on abelian categories.}

In this section we recall from [10, 59] the notion of a categorical action of $\mathfrak{g}$. It consists of the data of functors $E_{i}, F_{i}$ lifting the Chevalley generators $e_{i}, f_{i}$ of $\mathfrak{g}$, together with an action of an affine Hecke algebra on $\left(\bigoplus_{i \in I} F_{i}\right)^{m}$.

1.3.1. Affine Hecke algebras and representation data. Let $\mathscr{C}$ be an abelian $R$-category and $v \in R^{\times}$.

Definition 1.2. A representation datum on $\mathscr{C}$ with parameter $v$ is a tuple $(E, F, X, T)$ where $E, F$ are bi-adjoint functors $\mathscr{C} \rightarrow \mathscr{C}$ and $X \in \operatorname{End}(F)^{\times}, T \in \operatorname{End}\left(F^{2}\right)$ are endomorphisms of functors satisfying the following conditions:

(a) $1_{F} T \circ T 1_{F} \circ 1_{F} T=T 1_{F} \circ 1_{F} T \circ T 1_{F}$,

(b) $\left(T+1_{F^{2}}\right) \circ\left(T-v 1_{F^{2}}\right)=0$,

(c) $T \circ\left(1_{F} X\right) \circ T=v X 1_{F}$.

This definition can also be formulated in terms of actions of affine Hecke algebras. For $m \geqslant 1$, let $\mathbf{H}_{R, m}^{v}$ be the affine Hecke algebra of type $A_{m-1}$ over $R$. It is generated by $T_{1}, \ldots, T_{m-1}, X_{1}^{ \pm 1}, \ldots, X_{m}^{ \pm 1}$ subject to the well-known relations. We will also set $\mathbf{H}_{R, 0}^{v}=R$.

Given $(E, F)$ a pair of biadjoint functors, and $X \in \operatorname{End}(F), T \in \operatorname{End}\left(F^{2}\right)$, the tuple $(E, F, X, T)$ is a representation datum if and only if for each $m \in \mathbb{N}$, the map

$$
\begin{aligned}
\phi_{F^{m}}: \mathbf{H}_{R, m}^{v} & \longmapsto \operatorname{End}\left(F^{m}\right) \\
X_{k} & \longmapsto 1_{F^{m-k}} X 1_{F^{k-1}} \\
T_{l} & \longmapsto 1_{F^{m-l-1}} T 1_{F^{l-1}}
\end{aligned}
$$

is a well-defined $R$-algebra homomorphism.

1.3.2. Categorical representations. We assume now that $R$ is a field and that $\mathscr{C}$ is Hom-finite. We fix a pair $(I, v)$ as in $\$ 1.2$ and we denote by $\mathfrak{g}=\mathfrak{g}_{I}$ the Lie algebra associated to that pair.

Definition $1.3([59)$. A $\mathfrak{g}$-representation on $\mathscr{C}$ consists of a representation datum $(E, F, X, T)$ on $\mathscr{C}$ and of a decomposition $\mathscr{C}=\bigoplus_{\omega \in \mathrm{X}} \mathscr{C}_{\omega}$. For each $i \in I$, let $F_{i}$, $E_{i}$ be the generalized $i$-eigenspaces of $X$ acting on $F, E$ respectively. We assume in addition that

(a) $F=\bigoplus_{i \in I} F_{i}$ and $E=\bigoplus_{i \in I} E_{i}$, 
(b) the action of $\left[E_{i}\right]$ and $\left[F_{i}\right]$ for $i \in I$ endow $[\mathscr{C}]$ with a structure of integrable $\mathfrak{g}$-module such that $[\mathscr{C}]_{\omega}=\left[\mathscr{C}_{\omega}\right]$,

(c) $E_{i}\left(\mathscr{C}_{\omega}\right) \subset \mathscr{C}_{\omega+\alpha_{i}}$ and $F_{i}\left(\mathscr{C}_{\omega}\right) \subset \mathscr{C}_{\omega-\alpha_{i}}$.

We say that the tuple $(E, F, X, T)$ and the decomposition $\mathscr{C}=\bigoplus_{\omega \in \mathrm{X}} \mathscr{C}_{\omega}$ is a $\mathfrak{g}$-categorification of the integrable $\mathfrak{g}$-module $[\mathscr{C}]$.

\subsection{Minimal categorical representations.}

For most of the results in the rest of Section 1 we will assume that $R$ is a field and that $I$ is finite. In particular $v \in R^{\times}$will be a root of unity.

Let $m \geqslant 0, v \in R^{\times}$and $\mathbf{H}_{R, m}^{v}$ be the affine Hecke algebra as defined in \$1.3.1. We fix a tuple $Q=\left(Q_{1}, \ldots, Q_{l}\right)$ in $\left(R^{\times}\right)^{l}$. The cyclotomic Hecke algebra $\mathbf{H}_{R, m}^{Q ; v}$ is the quotient of $\mathbf{H}_{R, m}^{v}$ by the two-sided ideal generated by $\prod_{i=1}^{l}\left(X_{1}-Q_{i}\right)$.

Assume now that $R$ is a field. Any finite dimensional $\mathbf{H}_{R, m}^{Q ; v}$-module $M$ is the direct sum of the weight subspaces

$$
M_{\nu}=\left\{v \in M \mid\left(X_{r}-i_{r}\right)^{d} v=0, r \in[1, m], d \gg 0\right\}, \quad \nu=\left(i_{1}, \ldots, i_{m}\right) \in R^{m} .
$$

Decomposing the regular module, we get a system of orthogonal idempotents $\left\{e_{\nu} ; \nu \in\right.$ $\left.R^{m}\right\}$ in $\mathbf{H}_{R, m}^{Q ; v}$ such that $e_{\nu} M=M_{\nu}$ for each $M$. The eigenvalues of $X_{r}$ are always of the form $Q_{i} v^{j}$ for some $i \in\{1, \ldots, l\}$ and $j \in \mathbb{Z}$. As a consequence, if we set $I=\bigcup Q_{i} v^{\mathbb{Z}}$, then $e_{\nu}=0$ unless $\nu \in I$. The pair $(I, v)$ satisfies the assumptions of $\$ 1.2$ and we can consider a corresponding Kac-Moody algebra $\mathfrak{g}_{I}$ and its root lattice $\mathrm{Q}_{I}$. Given $\alpha \in \mathrm{Q}_{I}^{+}$of height $m$, let $e_{\alpha}=\sum_{\nu} e_{\nu}$ where the sum runs over the set of all tuples such that $\sum_{r=1}^{m} \alpha_{i_{r}}=\alpha$. The nonzero $e_{\alpha}$ 's are the primitive central idempotents in $\mathbf{H}_{R, m}^{Q ; v}$.

To the dominant weight $\Lambda_{Q}=\sum_{i=1}^{l} \Lambda_{Q_{i}}$ of $\mathfrak{g}_{I}$ and to any $\alpha \in \mathrm{Q}_{I}^{+}$we associate the following abelian categories:

$$
\mathscr{L}\left(\Lambda_{Q}\right)=\bigoplus_{m \in \mathbb{N}} \mathbf{H}_{R, m}^{Q ; v} \text {-mod and } \mathscr{L}\left(\Lambda_{Q}\right)_{\Lambda_{Q}-\alpha}=e_{\alpha} \mathbf{H}_{R, m}^{Q ; v}-\bmod .
$$

For any $m<n$, the $R$-algebra embedding of the affine Hecke algebras $\mathbf{H}_{R, m}^{v} \hookrightarrow \mathbf{H}_{R, n}^{v}$ given by $T_{i} \mapsto T_{i}$ and $X_{j} \mapsto X_{j}$ induces an embedding $\mathbf{H}_{R, m}^{Q ; v} \hookrightarrow \mathbf{H}_{R, n}^{Q ; v}$. The $R$ algebra $\mathbf{H}_{R, n}^{Q ; v}$ is free as a left and as a right $\mathbf{H}_{R, m}^{Q ; v}$-module. This yields a pair of exact adjoint functors $\left(\operatorname{Ind}_{m}^{n}\right.$, $\operatorname{Res}_{m}^{n}$ ) between $\mathbf{H}_{R, n}^{Q ; v}$-mod and $\mathbf{H}_{R, m}^{Q ; v}$-mod. They induce endofunctors $E$ and $F$ of $\mathscr{L}\left(\Lambda_{Q}\right)$ by $E=\bigoplus_{m \in \mathbb{N}} \operatorname{Res}_{m}^{m+1}$ and $F=\bigoplus_{m \in \mathbb{N}} \operatorname{Ind}_{m}^{m+1}$. The right multiplication on $\mathbf{H}_{R, m+1}^{Q ; v}$ by $X_{m+1}$ yields an endomorphism of the functor 
$\operatorname{Ind}_{m}^{m+1}$. The right multiplication by $T_{m+1}$ yields an endomorphism of $\operatorname{Ind}_{m}^{m+2}$. We define $X \in \operatorname{End}(F)$ and $T \in \operatorname{End}\left(F^{2}\right)$ by $X=\bigoplus_{m} X_{m+1}$ and $T=\bigoplus_{m} T_{m+1}$.

This construction yields a categorification of the simple highest module $\mathbf{L}\left(\Lambda_{Q}\right)$ of $\mathfrak{g}_{I}$. Indeed, a theorem of Kang and Kashiwara implies that this holds in the more general setting of cyclotomic quiver Hecke algebras of arbitrary type.

Theorem 1.4 ([46], [49]).

(a) The endofunctors $E$ and $F$ of $\mathscr{L}\left(\Lambda_{Q}\right)$ are biadjoint.

(b) The tuple $(E, F, X, T)$ and the decomposition $\mathscr{L}\left(\Lambda_{Q}\right)=\bigoplus_{\omega \in \mathrm{X}} \mathscr{L}\left(\Lambda_{Q}\right)_{\omega}$ is a $\mathfrak{g}_{I}$-categorification of $\mathbf{L}\left(\Lambda_{Q}\right)$.

This categorical representation is called the minimal categorical $\mathfrak{g}_{I}$-representation of highest weight $\Lambda_{Q}$.

The $\mathfrak{g}_{I}$-modules we are interested in are direct sums of various irreducible highest weight modules $\mathbf{L}\left(\Lambda_{Q}\right)$. Let $(I, v)$ as in $₫ 1.2$, and $\mathfrak{g}=\mathfrak{g}_{I}$ be a corresponding KacMoody algebra. Let $(E, F, X, T)$ be a $\mathfrak{g}$-representation on an abelian $R$-category $\mathscr{C}$. We want to relate $\mathscr{C}$ to minimal categorical $\mathfrak{g}_{I}$-representations. To do that, recall that for any $m \geqslant 0$ we have an $R$-algebra homomorphism $\phi_{F^{m}}: \mathbf{H}_{R, m}^{Q ; v} \longrightarrow \operatorname{End}\left(F^{m}\right)^{\mathrm{op}}$. Given an object $M$ in $\mathscr{C}$, it specializes to an $R$-algebra homomorphism

$$
\mathbf{H}_{R, m}^{Q ; v} \longrightarrow \operatorname{End}\left(F^{m} M\right)^{\mathrm{op}}=: \mathscr{H}\left(F^{m} M\right) \text {. }
$$

Proposition 1.5 ([59]). Assume that the simple roots are linearly independent in X. Let $(E, F, X, T)$ be a representation of $\mathfrak{g}$ in a abelian $R$-category $\mathscr{C}$, and $M \in \mathscr{C}_{\omega}$. Assume that $E M=0$ and $\operatorname{End}_{\mathscr{C}}(M)=R$. Then

(a) $\omega \in \mathrm{X}^{+}$is an integral dominant weight,

(b) if we write $\Lambda_{Q}=\sum_{i \in I}\left\langle\alpha_{i}^{\vee}, \omega\right\rangle \Lambda_{i}=\sum_{p=1}^{l} \Lambda_{Q_{p}}$ for some $Q=\left(Q_{1}, \ldots, Q_{l}\right) \in I^{l}$ and $l \geqslant 1$, then for all $m \geq 0$ the map $\phi_{F^{m}}$ factors to an $R$-algebra isomorphism

$$
\mathbf{H}_{R, m}^{Q ; v} \stackrel{\sim}{\longrightarrow} \mathscr{H}\left(F^{m} M\right) .
$$

\subsection{Crystals.}

We start by a review of Kashiwara's theory of perfect bases and crystals. We will be working with the Kac-Moody algebra $\mathfrak{g}$ coming from a pair $(I, v)$ as in $\$ 1.2$.

Definition 1.6. An abstract crystal is a set $B$ together with maps wt $: B \rightarrow \mathrm{P}, \varepsilon_{i}$, $\varphi_{i}: B \rightarrow \mathbb{Z} \sqcup\{-\infty\}$ and $\widetilde{e}_{i}, \widetilde{f}_{i}: B \rightarrow B \sqcup\{0\}$ for all $i \in I$ satisfying the following properties:

(a) $\varphi_{i}(b)=\varepsilon_{i}(b)+\left\langle\alpha_{i}^{\vee}, \operatorname{wt}(b)\right\rangle$, 
(b) $\operatorname{wt}\left(\widetilde{e}_{i} b\right)=\operatorname{wt}(b)+\alpha_{i}$ and $\operatorname{wt}\left(\widetilde{f}_{i} b\right)=\operatorname{wt}(b)-\alpha_{i}$,

(c) $b=\widetilde{e}_{i} b^{\prime}$ if and only if $\widetilde{f}_{i} b=b^{\prime}$, where $b, b^{\prime} \in B, i \in I$,

(d) if $\varphi_{i}(b)=-\infty$, then $\widetilde{e}_{i} b=\widetilde{f}_{i} b=0$,

(e) if $b \in B$ and $\widetilde{e_{i}} b \in B$, then $\varepsilon_{i}\left(\widetilde{e}_{i} b\right)=\varepsilon_{i}(b)-1$ and $\varphi_{i}\left(\widetilde{e}_{i} b\right)=\varphi_{i}(b)+1$,

(f) if $b \in B$ and $\widetilde{f}_{i} b \in B$, then $\varepsilon_{i}\left(\widetilde{f}_{i} b\right)=\varepsilon_{i}(b)+1$ and $\varphi_{i}\left(\widetilde{f}_{i} b\right)=\varphi_{i}(b)-1$.

Note that by (a), the map $\varphi_{i}$ is entirely determined by $\varepsilon_{i}$ and wt. We may therefore omit $\varphi_{i}$ in the data of an abstract crystal and denote it by $\left(B, \widetilde{e}_{i}, \widetilde{f}_{i}, \varepsilon_{i}\right.$, wt $)$.

An isomorphism between crystals $B_{1}, B_{2}$ is a bijection $\psi: B_{1} \sqcup\{0\} \longrightarrow B_{2} \sqcup\{0\}$ such that $\psi(0)=0$ which commutes with wt, $\varepsilon_{i}, \varphi_{i} \widetilde{f}_{i}, \widetilde{e}_{i}$.

Let $V_{u}$ be an integrable $U_{u}(\mathfrak{g})$-module in $\mathcal{O}_{u}^{\text {int }}$. Let $V_{A}$ be an integral form of $V_{u}$. A lower crystal lattice in $V_{u}$ is a free $\mathbb{C}[u]$-submodule $\mathcal{L}$ of $V_{A}$ such that $V_{A}=A \mathcal{L}$, $\mathcal{L}=\bigoplus_{\lambda \in \mathrm{X}} \mathcal{L}_{\lambda}$ with $\mathcal{L}_{\lambda}=\mathcal{L} \cap\left(V_{A}\right)_{\lambda}$ and $\mathcal{L}$ is preserved by the lower Kashiwara crystal operators $\widetilde{e}_{i}^{\mathrm{lw}}, \widetilde{f}_{i}^{\mathrm{lw}}$ on $V_{u}$. A lower crystal basis of $V_{u}$ is a pair $(\mathcal{L}, B)$ where $\mathcal{L}$ is a lower crystal lattice of $V_{u}$ and $B$ is a basis of $\mathcal{L} / u \mathcal{L}$ such that we have $B=\bigsqcup_{\lambda \in \mathrm{X}} B_{\lambda}$ where $B_{\lambda}=B \cap\left(\mathcal{L}_{\lambda} / u \mathcal{L}_{\lambda}\right), \widetilde{e}_{i}^{\mathrm{lw}}(B), \widetilde{f}_{i}^{\mathrm{lw}}(B) \subset B \sqcup\{0\}$ and $b^{\prime}=\widetilde{f}_{i}^{\mathrm{lw}} b$ if and only if $b=\widetilde{e}_{i}^{\mathrm{lw}} b^{\prime}$ for each $b, b^{\prime} \in B$. A lower global basis of $V_{u}$ (or a canonical basis) is an $A$-basis $\mathbf{B}$ of $V_{A}$ such that the lattice $\mathcal{L}=\bigoplus_{b \in \mathbf{B}} \mathbb{C}[u] b$ and the basis $B=\{b \bmod u \mathcal{L} \mid b \in \mathbf{B}\}$ of $\mathcal{L} / u \mathcal{L}$ form a lower crystal basis. One defines in a similar way an upper crystal lattice, an upper crystal basis and an upper global basis (or a dual canonical basis) using the upper Kashiwara crystal operators $\widetilde{e}_{i}^{\text {up }}, \widetilde{f}_{i}^{\text {up }}$ on $V_{u}$, see, e.g., [47, def. 4.1,4.2]. Any $U_{u}(\mathfrak{g})$-module in $\mathcal{O}_{u}^{\text {int }}$ admits a lower crystal, an upper crystal and a global basis.

If $(\mathcal{L}, B),\left(\mathcal{L}^{\vee}, B^{\vee}\right)$ are lower, upper crystal bases, then $\left(B, \widetilde{e}_{i}^{\text {lw }}, \widetilde{f}_{i}^{\text {lw }}\right),\left(B^{\vee}, \widetilde{e}_{i}^{\text {up }}, \widetilde{f}_{i}^{\text {up }}\right)$ are abstract crystals.

Let $E_{i}, F_{i}, u^{h}$ with $i \in I, h \in \mathrm{X}^{\vee}$, be the standard generators of $U_{u}(\mathfrak{g})$. There exists a unique non-degenerate symmetric bilinear form $(\bullet, \bullet)$ on the module $\mathbf{L}_{u}(\Lambda)$ with highest weight vector $|\Lambda\rangle$ satisfying

- $(|\Lambda\rangle,|\Lambda\rangle)=1$,

- $\left(E_{i} x, y\right)=\left(x, F_{i} y\right),\left(F_{i} x, y\right)=\left(x, E_{i} y\right),\left(u^{h} x, y\right)=\left(x, u^{h} y\right)$,

- $\left(\mathbf{L}_{u}(\Lambda)_{\lambda}, \mathbf{L}_{u}(\Lambda)_{\mu}\right)=0$ if $\lambda \neq \mu$.

If $(\mathcal{L}, B)$ is a lower crystal basis of $\mathbf{L}_{u}(\Lambda)$ then the pair $\left(\mathcal{L}^{\vee}, B^{\vee}\right)$ such that $\mathcal{L}^{\vee}=\{x \in$ $\left.\mathbf{L}_{u}(\Lambda) \mid(x, \mathcal{L}) \subset \mathbb{C}[u]\right\}$ and $B^{\vee}$ is the basis of $\mathcal{L}^{\vee} / u \mathcal{L}^{\vee}$ which is dual to $B$ with respect to the non-degenerate bilinear form $\mathcal{L}^{\vee} / u \mathcal{L}^{\vee} \times \mathcal{L} / u \mathcal{L} \rightarrow \mathbb{C}$ induced by $(\bullet, \bullet)$, is an upper crystal basis. Further, taking a basis element in $B$ to the dual basis element in $B^{\vee}$ is a crystal isomorphism $\left(B, \widetilde{e}_{i}^{\mathrm{lw}}, \widetilde{f}_{i}^{\mathrm{lw}}\right) \rightarrow\left(B^{\vee}, \widetilde{e}_{i}^{\text {up }}, \widetilde{f}_{i}^{\text {up }}\right)$. Therefore, if $\mathbf{B}$ is a lower global basis of $\mathbf{L}_{u}(\Lambda)$ then the dual basis $\mathbf{B}^{\vee}$ with respect to the non-degenerate bilinear form $(\bullet, \bullet)$ is an upper global basis and the corresponding abstract crystals $\left(B, \widetilde{e}_{i}^{\mathrm{lw}}, \widetilde{f}_{i}^{\mathrm{lw}}\right)$ and $\left(B^{\vee}, \widetilde{e}_{i}^{\mathrm{up}}, \widetilde{f}_{i}^{\mathrm{up}}\right)$ are canonically isomorphic. 


\subsection{Perfect bases.}

The crystals that we will consider in this paper all come from particular bases of $\mathfrak{g}_{I}$-modules called perfect bases. Let us define them. Let $V \in \mathcal{O}^{\text {int }}$ be an integrable highest weight $\mathfrak{g}_{I}$-module. For each $i \in I$ and $x \in V$ we define

$$
\ell_{i}(x)=\max \left\{k \in \mathbb{N} \mid e_{i}^{k} x \neq 0\right\}=\min \left\{k \in \mathbb{N} \mid e_{i}^{k+1} x=0\right\}
$$

with the convention that $\ell_{i}(0)=-\infty$. For each integer $k$, we also consider the vector spaces

$$
V_{i}^{\leqslant k}=\left\{x \in V \mid \ell_{i}(x) \leqslant k\right\}, \quad V^{\leqslant k}=\bigcap_{i \in I} V_{i}^{\leqslant k}, \quad V_{i}^{k}=V_{i}^{\leqslant k} / V_{i}^{<k} .
$$

Definition 1.7. A basis $B$ of $V$ is perfect if

(a) $B=\bigsqcup_{\mu \in \mathrm{X}_{I}} B_{\mu}$ where $B_{\mu}=B \cap V_{\mu}$,

(b) for any $i \in I$, there is a map $\mathbf{e}_{i}: B \rightarrow B \sqcup\{0\}$ such that for any $b \in B$, we have

(i) if $\ell_{i}(b)=0$, then $\mathbf{e}_{i} b=0$,

(ii) if $\ell_{i}(b)>0$, then $\mathbf{e}_{i} b \in B$ and $e_{i} b \in \mathbb{C}^{\times} \mathbf{e}_{i} b+V_{i}^{<\ell_{i}(b)-1}$,

(c) if $\mathbf{e}_{i} b=\mathbf{e}_{i} b^{\prime} \neq 0$ for $b, b^{\prime} \in B$, then $b=b^{\prime}$.

Any $\mathfrak{g}$-module in $\mathcal{O}^{\text {int }}$ admits a perfect basis. More precisely, we have the following.

Proposition 1.8. If $V$ is an integrable $\mathfrak{g}$-module in $\mathcal{O}^{\text {int }}$ with a quantum deformation $V_{u}$, then the specialization at $u=1$ of an upper global basis of $V_{u}$ is a perfect basis of $V$.

To any categorical representation we associate a perfect basis as in [61, prop. 6.2]. More precisely, let $R$ be a field (of any characteristic) and consider a $\mathfrak{g}$-representation on an abelian artinian $R$-category $\mathscr{C}$. Then, for each $i \in I$ we define the maps

$$
\begin{array}{ll}
\widetilde{E}_{i}: \operatorname{Irr}(\mathscr{C}) \rightarrow \operatorname{Irr}(\mathscr{C}) \sqcup\{0\}, & {[L] \mapsto\left[\operatorname{soc}\left(E_{i}(L)\right)\right],} \\
\widetilde{F}_{i}: \operatorname{Irr}(\mathscr{C}) \rightarrow \operatorname{Irr}(\mathscr{C}) \sqcup\{0\}, & {[L] \mapsto\left[\operatorname{hd}\left(F_{i}(L)\right)\right] .}
\end{array}
$$

Proposition 1.9. The tuple $\left(\operatorname{Irr}(\mathscr{C}), \widetilde{E}_{i}, \widetilde{F}_{i}\right)$ defines a perfect basis of $[\mathscr{C}]$.

We now recall how to construct an abstract crystal from a perfect basis $B$. We set $\widetilde{e}_{i}=\mathbf{e}_{i}$. For all $b \in B$ we set $\widetilde{f}_{i} b=b^{\prime}$ if $\mathbf{e}_{i} b^{\prime}=b$ for some $b^{\prime} \in B$, and 0 otherwise. Then it follows easily from the definition that $\left(B, \widetilde{e}_{i}, \widetilde{f}_{i}, \ell_{i}\right.$, wt $)$ is an abstract crystal.

We finish this section with a result which will be important to identify the crystal obtained by the categorification with the crystal of some Fock space. For each $i \in I$ and $k \in \mathbb{N}$, we set $B^{\leqslant k}=V^{\leqslant k} \cap B$ and $B_{i}^{\leqslant k}=V_{i}^{\leqslant k} \cap B$.

Proposition 1.10. Let $B$ and $B^{\prime}$ be perfect bases of $V \in \mathcal{O}^{\text {int }}$. 
(a) $B^{\leqslant k}$ and $B_{i}^{\leqslant k}$ are bases of $V^{\leqslant k}$ and $V_{i}^{\leqslant k}$.

(b) Assume that there is a bijection $\varphi: B \rightarrow B^{\prime}$ and a partial order $\leqslant$ on $B$ such that $\varphi(b) \in b+\sum_{c>b} \mathbb{C} c$ for each $b \in B$. Then the map $\varphi$ is a crystal isomorphism $B \stackrel{\sim}{\rightarrow} B^{\prime}$.

\subsection{Derived equivalences.}

Given $V$ an integrable $\mathfrak{g}$-module and $i \in I$, one can consider the action of the simple reflection $s_{i}=\exp \left(-f_{i}\right) \exp \left(e_{i}\right) \exp \left(-f_{i}\right)$ on $V$. For each weight $\omega \in \mathrm{X}$, this action maps a weight space $V_{\omega}$ to $V_{s_{i}(\omega)}$ with $s_{i}(\omega)=\omega-\left\langle\alpha_{i}^{\vee}, \omega\right\rangle \alpha_{i}$. If $\mathscr{C}$ is a categorification of $V$, then it restricts to an $\mathfrak{s l}_{2}(\mathbb{C})$-categorification in the sense of Chuang-Rouquier. In particular, the simple objects are weight vectors for the categorical $\mathfrak{s l}_{2}(\mathbb{C})$-action. Thus, the theory of Chuang-Rouquier can be applied and [10, thm. 6.6] implies that $s_{i}$ can be lifted to a derived equivalence $\Theta_{i}$ of $\mathscr{C}$.

Theorem 1.11. Assume that $R$ is a field. Let $(E, F, X, T)$ be a representation of $\mathfrak{g}$ in an abelian $R$-category $\mathscr{C}$, and $i \in I$. Then there exists a derived self-equivalence $\Theta_{i}$ of $\mathscr{C}$ which restricts to derived equivalences $\Theta_{i}: D^{b}\left(\mathscr{C}_{\omega}\right) \stackrel{\sim}{\longrightarrow} D^{b}\left(\mathscr{C}_{s_{i}(\omega)}\right)$ for all weightn $\omega \in \mathrm{X}$. Furthermore, $\left[\Theta_{i}\right]=s_{i}$ as a linear map of $[\mathscr{C}]$.

\section{Representations on Fock spaCeS}

Let $R$ be a noetherian commutative domain with unit. As in \$1.2, we fix an element $v \in R^{\times}$and a subset $I$ of $R^{\times}$which is stable by multiplication by $v$ and $v^{-1}$. We explained in $\$ 1.2 .1$ how one can associate a Lie algebra $\mathfrak{g}=\mathfrak{g}_{I}$ to this data. In this section we recall the construction of (charged) Fock spaces which are particular integrable representations of $\mathfrak{g}$.

\subsection{Combinatorics of $l$-partitions.}

2.1.1. Partitions and l-partitions. A partition of $n$ is a non-increasing sequence of non-negative integers $\lambda=\left(\lambda_{1} \geqslant \lambda_{2} \geqslant \cdots\right)$ whose terms add up to $n$. We denote by $\mathscr{P}_{n}$ the set of partitions of $n$ and by $\mathscr{P}=\bigsqcup_{n} \mathscr{P}_{n}$ be the set of all partitions. Given a partition $\lambda$, we write $|\lambda|$ for the weight of $\lambda$. We associate to $\lambda=\left(\lambda_{1}, \lambda_{2}, \ldots\right)$ the Young diagram $Y(\lambda)$ defined by $Y(\lambda)=\left\{(x, y) \in \mathbb{Z}_{>0} \times \mathbb{Z}_{>0} \mid y \leqslant \lambda_{x}\right\}$. It may be visualised by an array of boxes in left justified rows with $\lambda_{x}$ boxes in the $x$-th row. If $\lambda, \mu$ are partitions of $n$ then we write $\lambda \geqslant \mu$ if for all $n \geqslant i \geqslant 1$ we have $\sum_{j=1}^{i} \lambda_{j} \geqslant \sum_{j=1}^{i} \mu_{j}$. This relation defines a partial order on $\mathscr{P}$ called the dominance order. Let $\lambda^{*}$ denote the partition dual (or conjugate) to $\lambda$. 
An $l$-partition of $n$ is an $l$-tuple of partitions whose weights add up to $n$. We denote by $\mathscr{P}_{n}^{l}$ be the set of $l$-partitions of $n$ and by $\mathscr{P}^{l}=\bigsqcup_{n} \mathscr{P}_{n}^{l}$ the set of all $l$-partitions. The Young diagram of the $l$-partition $\lambda=\left(\lambda^{1}, \ldots, \lambda^{l}\right)$ is the set $Y(\lambda)=$ $\bigsqcup_{p=1}^{l} Y\left(\lambda^{p}\right) \times\{p\}$. Its weight is the integer $|\lambda|=\sum_{p}\left|\lambda^{p}\right|$.

2.1.2. Residues and content. We fix $Q=\left(Q_{1}, \ldots, Q_{l}\right) \in I^{l}$. Let $\lambda$ be an $l$-partition and $A=(x, y, p)$ be a node in $Y(\lambda)$. The $(Q, v)$-shifted residue of the node $A$ is the element of $I$ given by $\operatorname{res}(A, Q)_{I}=v^{y-x} Q_{p}$. Let $n_{i}(\lambda, Q)_{I}$ be the number of nodes of $(Q, v)$-shifted residue $i$ in $Y(\lambda)$. If $\lambda, \mu$ are $l$-partitions such that $|\mu|=|\lambda|+1$ we write $\operatorname{res}(\mu-\lambda, Q)_{I}=i$ if $Y(\mu)$ is obtained by adding a node of $(Q, v)$-shifted residue $i$ to $Y(\lambda)$. A charge of the tuple $Q=\left(Q_{1}, \ldots, Q_{l}\right)$ is an $l$-tuple of integers $s=\left(s_{1}, \ldots, s_{l}\right)$ such that $Q_{p}=v^{s_{p}}$ for all $p=1, \ldots, l$. Conversely, given $I \subset R^{\times}$and $v \in R^{\times}$as in $\$ 1.2$, any $\ell$-tuple of integers $s \in \mathbb{Z}^{l}$ defines a tuple $Q=\left(v^{s_{1}}, \ldots, v^{s_{l}}\right)$ with charge $s$. The $s$-shifted content of the box $A=(x, y, p)$ is the integer $\operatorname{ct}^{s}(A)=s_{p}+y-x$. It is related to the residue of $A$ by the formula $\operatorname{res}(A, Q)_{I}=v^{\operatorname{ct}^{s}(A)}$. We will also write $p(A)=p$. We will call charged l-partition a pair $(\mu, s)$ in $\mathscr{P}^{l} \times \mathbb{Z}^{l}$.

2.1.3. $l$-cores and $l$-quotients. We start with the case $l=1$. The set of $\beta$-numbers of a charged partition $(\lambda, d) \in \mathscr{P} \times \mathbb{Z}$ is the set given by $\beta_{d}(\lambda)=\left\{\lambda_{u}+d+1-u \mid u \geqslant 1\right\}$. The charged partition $(\lambda, d)$ is uniquely determined by the set $\beta_{d}(\lambda)$.

For any positive integer $e$, an $e$-hook of $(\lambda, d)$ is a pair $(x, x+e)$ such that $x+e \in$ $\beta_{d}(\lambda)$ and $x \notin \beta_{d}(\lambda)$. Removing the $e$-hook $(x, x+e)$ corresponds to replacing $x+e$ with $x$ in $\beta_{d}(\lambda)$. We say that the charged partition $(\lambda, d)$ is an $e$-core if it does not have any $e$-hook. This does not depend on $d$.

Next, we construct a bijection $\tau_{l}: \mathscr{P} \times \mathbb{Z} \rightarrow \mathscr{P}^{l} \times \mathbb{Z}^{l}$. It takes the pair $(\lambda, d)$ to $(\mu, s)$, where $\mu=\left(\mu^{1}, \ldots, \mu^{l}\right)$ is an $l$-partition and $s=\left(s_{1}, \ldots, s_{l}\right)$ is a $l$-tuple in $\mathbb{Z}^{l}(d)=\left\{s \in \mathbb{Z}^{l} \mid s_{1}+\cdots+s_{l}=d\right\}$. The bijection is uniquely determined by the relation $\beta_{d}(\lambda)=\bigsqcup_{p=1}^{l}\left(p-l+l \beta_{s_{p}}\left(\mu^{p}\right)\right)$.

The bijection $\tau_{l}$ takes the pair $(\lambda, 0)$ to $\left(\lambda^{[l]}, \lambda_{[l]}\right)$, where $\lambda^{[l]}$ is the $l$-quotient of $\lambda$ and $\lambda_{[l]}$ lies in $\mathbb{Z}^{l}(0)$. Since $\lambda$ is an $l$-core if and only if $\lambda^{[l]}=\emptyset$, this bijection identifies the set of $l$-cores and $\mathbb{Z}^{l}(0)$. We define the $l$-weight $w_{l}(\lambda):=\left|\lambda^{[l]}\right|$ of the partition $\lambda$ to be the weight of its $l$-quotient.

We will mostly consider the bijection $\tau_{l}$ for $l=2$. In particular, a 2-core is either $\Delta_{0}=\emptyset$ or a triangular partition $\Delta_{t}=(t, t-1, \ldots, 1)$ with $t \in \mathbb{N}$. We abbreviate $\sigma_{t}=\left(\Delta_{t}\right)_{[2]}$, and we write $\sigma_{t}=\left(\sigma_{1}, \sigma_{2}\right)$. We have

$$
\sigma_{t}= \begin{cases}(-t / 2, t / 2) & \text { if } t \text { is even } \\ ((1+t) / 2,-(1+t) / 2) & \text { if } t \text { is odd }\end{cases}
$$


For each bipartition $\mu$, let $\varpi_{t}(\mu)$ denote the unique partition with 2-quotient $\mu$ and 2 -core $\Delta_{t}$. Thus, the bijection $\tau_{2}$ maps $\left(\varpi_{t}(\mu), 0\right)$ to the pair $\left(\mu, \sigma_{t}\right)$.

\subsection{Fock spaces.}

For a reference for the results presented in this section, see for example [65], [66]. Let $Q=\left(Q_{1}, \ldots, Q_{l}\right) \in I^{l}$. It defines an integral dominant weight $\Lambda_{Q}=\sum_{p=1}^{l} \Lambda_{Q_{p}} \in$ $\mathrm{P}^{+}$. The Fock space $\mathbf{F}(Q)_{I}$ is the $\mathbb{C}$-vector space with basis $\left\{|\lambda, Q\rangle_{I} \mid \lambda \in \mathscr{P}^{l}\right\}$ called the standard monomial basis, and action of $e_{i}, f_{i}$ for all $i \in I$ given by

$$
f_{i}\left(|\lambda, Q\rangle_{I}\right)=\sum_{\mu}|\mu, Q\rangle_{I}, \quad e_{i}\left(|\mu, Q\rangle_{I}\right)=\sum_{\lambda}|\lambda, Q\rangle_{I}
$$

where the sums run over all partitions such that $\operatorname{res}(\mu-\lambda, Q)_{I}=i$. This endows $\mathbf{F}(Q)_{I}$ with a structure of $\mathfrak{g}^{\prime}$-module. The Fock space $\mathbf{F}(Q)_{I}$ can also be equipped with a symmetric non-degenerate bilinear form $\langle\bullet, \bullet\rangle_{I}$ for which the standard monomial basis is orthonormal. To avoid cumbersome notation, we shall omit the subscript $I$ when not necessary. It is easy to see that every element of the standard monomial basis is a weight vector.

The $\mathfrak{g}^{\prime}$-submodule of $\mathbf{F}(Q)$ generated by $|\emptyset, Q\rangle$ is isomorphic to $\mathbf{L}\left(\Lambda_{Q}\right)$. Furthermore, if $I=A_{\infty}$, then $\mathbf{F}(Q)=\mathbf{L}\left(\Lambda_{Q}\right)$. Using the minimal categorification $\mathscr{L}\left(\Lambda_{Q}\right)$ of $\mathbf{L}\left(\Lambda_{Q}\right)$, the map $\mathbf{L}\left(\Lambda_{Q}\right) \rightarrow \mathbf{F}(Q)$ can be made more explicit.

To explain this, let us first recall briefly the definition of the Specht modules. Assume that $R$ has characteristic 0 and contains a primitive $l$-th $\operatorname{root} \zeta$ of 1 , so $R$ is a splitting field of the complex reflection group $G(l, 1, m)$. Let $\operatorname{Irr}\left(R \mathfrak{S}_{m}\right)=\left\{\phi_{\lambda} \mid \lambda \in\right.$ $\left.\mathscr{P}_{m}\right\}$ be the standard labelling of the characters of the symmetric group. Then

$$
\operatorname{Irr}(R G(l, 1, m))=\left\{\mathscr{X}_{\lambda} \mid \lambda \in \mathscr{P}_{m}^{l}\right\}
$$

is the labelling of the simple modules such that $\mathscr{X}_{\lambda}$ is induced from the $G\left(l, 1,\left|\lambda_{1}\right|\right) \times$ $\ldots \times G\left(l, 1,\left|\lambda_{l}\right|\right)$-module

$$
\phi_{\lambda^{(1)}} \chi^{0} \otimes \phi_{\lambda^{(2)}} \chi^{1} \otimes \cdots \otimes \phi_{\lambda^{(l)}} \chi^{l-1} .
$$

Here, we denote by $\chi^{p}$ the one-dimensional module of the $\left|\lambda_{p}\right|$-th cartesian power of the cyclic group $G(l, 1,1)$ given by the $p$-th power of the determinant, see, e.g., [33, sec. 5.1.3]. Recall that (for every field $R$ ) the $R$-algebra $\mathbf{H}_{R, m}^{Q ; v}$ is split and that it is semisimple if and only if we have, see, e.g., [58, sec. 3.2],

$$
\prod_{i=1}^{m}\left(1+v+\cdots+v^{i-1}\right) \prod_{a<b} \prod_{-m<r<m}\left(v^{r} Q_{a}-Q_{b}\right) \neq 0 .
$$


Thus, by Tits' deformation theorem, under the specialization $v \mapsto 1$ and $Q_{p} \mapsto \zeta^{p-1}$, the labelling of $\operatorname{Irr}(R G(l, 1, m))$ yields a canonical labelling

$$
\operatorname{Irr}\left(\mathbf{H}_{R, m}^{Q ; v}\right)=\left\{S(\lambda)_{R}^{Q ; v} \mid \lambda \in \mathscr{P}_{m}^{l}\right\}
$$

Now, if $R$ is a commutative domain with fraction field $K$ of characteristic 0 as above, we define the $\mathbf{H}_{R, m}^{Q ; v}$-module $S(\lambda)_{R}^{Q, v}$ as in [60, sec. 2.4.3] or [33, sec. 5.3], using $S(\lambda)_{K}^{Q ; v}$ and the dominance order on $\mathscr{P}_{m}^{l}$, and if $\theta: R \rightarrow \mathrm{k}$ is a ring homomorphism such that $\mathrm{k}$ is the fraction field of $\theta(R)$ we set $S(\lambda)_{\mathrm{k}}^{Q ; v}=\mathrm{k} S(\lambda)_{R}^{Q, v}$.

Proposition 2.1. Let $R$ be a field of characteristic 0 which contains a primitive l-th root of 1 . The composition $\left[\mathscr{L}\left(\Lambda_{Q}\right)\right] \stackrel{\sim}{\rightarrow} \mathbf{L}\left(\Lambda_{Q}\right) \rightarrow \mathbf{F}(Q)$ obtained from Theorem 1.4 sends the class of $S(\lambda)_{R}^{Q, v}$ to the standard monomial $|\lambda, Q\rangle$.

For each $p=1, \ldots, l$, let $I_{p}$ be the subquiver of $I$ corresponding to the subset $v^{\mathbb{Z}} Q_{p}$ of $I$. We define a relation on $\{1, \ldots, l\}$ by $i \sim j \Longleftrightarrow I_{i}=I_{j}$. Let $\Omega=\{1, \ldots, l\} / \sim$ be the set of equivalence classes for this relation. Given $p \in \Omega$, we denote by $Q_{p}$ the tuple of $\left(Q_{i_{1}}, \ldots, Q_{i_{r}}\right)$ where $\left(i_{1}, \ldots, i_{r}\right)$ is the ordered set of elements in $p$. The decomposition $I=\bigsqcup_{p \in \Omega} I_{p}$ yields a canonical decomposition of Lie algebras $\mathfrak{g}_{I}^{\prime}=$ $\bigoplus_{p \in \Omega} \mathfrak{g}_{I_{p}}^{\prime}$. The corresponding decomposition of Fock spaces is given in the following proposition.

Proposition 2.2. The map $|\lambda, Q\rangle_{I} \longmapsto \otimes_{p \in \Omega}\left|\lambda^{p}, Q_{p}\right\rangle_{I_{p}}$ yields an isomorphism of $\mathfrak{g}_{I}^{\prime}$-modules $\mathbf{F}(Q)_{I} \stackrel{\sim}{\longrightarrow} \bigotimes_{p \in \Omega} \mathbf{F}\left(Q_{p}\right)_{I_{p}}$.

\subsection{Charged Fock spaces.}

A charged Fock space is a pair $\mathbf{F}(s)=(\mathbf{F}(Q), s)$ such that $s \in \mathbb{Z}^{l}$ is a charge of $Q$, that is $Q=\left(v^{s_{1}}, \ldots, v^{s_{l}}\right)$. Throughout this section, we will always assume that $I$ is either of type $A_{\infty}$ or a cyclic quiver. For more general quivers we can invoke Proposition 2.2 to reduce to that case.

2.3.1. The $\mathfrak{g}$-action on the Fock space. The action of $\mathfrak{g}^{\prime}$ on $\mathbf{F}(Q)$ can be extended to an action of $\mathfrak{g}$ when $Q$ admits a charge $s$. We describe this action in the case where $v$ has finite order $e$, and $l=1$. In that case $I=v^{\mathbb{Z}}$ is isomorphic to the cyclic quiver $A_{e-1}^{(1)}$ and the charge $s$ is just an integer $d \in \mathbb{Z}$ such that $Q=v^{d}$. If we fix the affine simple root to be $\alpha_{1}$, then $\mathrm{X}=\mathrm{P} \oplus \mathbb{Z} \delta$ and $\mathrm{X}^{\vee}=\mathrm{Q}^{\vee} \oplus \mathbb{Z} \partial$ with $\delta=\sum_{i \in I} \alpha_{i}$ and $\partial=\Lambda_{1}^{\vee}$ (see Example 1.1 for more details).

We define the integer

$$
\Delta(d, e)=(\bar{d}(1-\bar{d} / e)+d(d / e-1)) / 2,
$$


where $\bar{d}$ is the residue of $d$ modulo $e$ in $[0, e-1]$. Then, the action of the derivation $\partial$ on $\mathbf{F}(d)=(\mathbf{F}(Q), d)$ is

$$
\partial(|\lambda, Q\rangle)=-\left(n_{1}(\lambda, Q)+\Delta(d, e)\right)|\lambda, Q\rangle .
$$

For this action the weight of a standard basis element is

$$
\operatorname{wt}(|\lambda, Q\rangle)=\Lambda_{Q}-\sum_{i \in I} n_{i}(\lambda, Q) \alpha_{i}-\Delta(d, e) \delta .
$$

We now describe the action of the affine Weyl group of $\mathfrak{g}$ on $\mathbf{F}(d)$. For $i \in I \backslash\{1\}$, we denote by $\alpha_{i}^{\mathrm{cl}}=2 \Lambda_{i}-\Lambda_{i v}-\Lambda_{i v^{-1}}$ and $\Lambda_{i}^{\mathrm{cl}}=\Lambda_{i}-\Lambda_{1}$ the $i$-th simple root and fundamental weight of $\mathfrak{s l}_{e}$. These (classical) simple roots span the lattice of classical roots $\mathrm{Q}^{\mathrm{cl}}$. The affine Weyl group of $\mathfrak{g}$ is $W=\mathfrak{S}_{I} \ltimes \mathrm{Q}^{\mathrm{cl}}$. It acts linearly on $\mathrm{X}$. We will denote by $t_{\gamma}$ the action of an element $\gamma \in \mathrm{Q}^{\text {cl }}$, i.e., for each $\alpha \in \mathrm{X}$ we set

$$
t_{\gamma}(\alpha)=\alpha+(\alpha: \delta) \gamma-(\alpha: \gamma) \delta-\frac{1}{2}(\alpha: \delta)(\gamma: \gamma) \delta
$$

where $(\bullet: \bullet)$ is the standard symmetric non-degenerate bilinear form on $\mathrm{X} \times \mathrm{X}$. Now, we consider the element $\pi_{s}=\sum_{i \in I}\left(s_{i}-s_{i v}\right) \Lambda_{i}$. If $s \in \mathbb{Z}^{I}(d)$, then $\pi_{s}-\Lambda_{Q}^{\mathrm{cl}} \in \mathrm{Q}^{\mathrm{cl}}$ and we can consider the corresponding operator $t_{\pi_{s}-\Lambda_{Q}^{\text {cl }}}$ on X.

Proposition 2.3. Let $\lambda$ and $\nu$ be two partitions. Let $s \in \mathbb{Z}^{I}(d)$ be such that $(\emptyset, s)=$ $\tau_{e}\left(\lambda_{[e]}, d\right)$, where $\lambda_{[e]}$ is the e-core of $\lambda$.

(a) The weight of $|\lambda, Q\rangle$ equals $\mathrm{wt}(|\lambda, Q\rangle)=t_{\pi_{s}-\Lambda_{Q}^{\mathrm{cl}}}\left(\Lambda_{Q}\right)-w_{e}(\lambda) \delta$.

(b) The weights of $|\lambda, Q\rangle$ and $|\nu, Q\rangle$ are $W$-conjugate if and only if $w_{e}(\lambda)=w_{e}(\nu)$.

In the particular case where the charge is zero, then $s=\lambda_{[e]}$, and

$$
\operatorname{wt}(|\lambda, 1\rangle)=t_{\pi_{\lambda_{[e]}}}\left(\Lambda_{1}\right)-w_{e}(\lambda) \delta .
$$

Therefore weight spaces are parametrized by pairs $(\nu, w)$ where $\nu$ is an $e$-core and $w$ is a non-negative integer. The basis element $|\lambda, 1\rangle$ is in the weight space corresponding to $\left(\lambda_{[e]}, w_{e}(\lambda)\right)$.

2.3.2. The crystal of the Fock space. We explain here how to associate an abstract crystal to a charged Fock space $\mathbf{F}(s)$. By Proposition 2.2, we can assume that $I$ is either cyclic or of type $A_{\infty}$. We assume that the reader is familiar with [65].

When $I$ has type $A_{\infty}$, Uglov's bases coincide with the standard monomial basis and the discussion is trivial in that case. We will therefore assume that $v$ has finite order $e$ and $I=v^{\mathbb{Z}}$, so that $I$ has type $A_{e-1}^{(1)}$. 
The $\mathfrak{g}$-module $\mathbf{F}(Q)$ admits a quantum deformation $\mathbf{F}_{u}(s)$ with an $A$-lattice $\mathbf{F}_{A}(s)$, which is a free $A$-module with basis $\left\{|\mu, s\rangle \mid \mu \in \mathscr{P}^{l}\right\}$. It is equipped with an integrable representation of $U_{A}(\mathfrak{g})$. Note that the action of the Chevalley generators $e_{i}$ and $f_{i}$ depends on the choice of the charge $s$. The representation of $\mathfrak{g}$ on $\mathbf{F}(s)$ given in $\$ 2.3 .1$ is recovered by specializing the parameter $u$ to 1 . Uglov has constructed a remarkable $A$-basis $\mathbf{B}_{u}^{+}(s)=\left\{\mathbf{b}_{u}^{+}(\mu, s) \mid \mu \in \mathscr{P}^{l}\right\}$ of $\mathbf{F}_{A}(s)$ which is a lower global basis for the representation of $U_{u}(\mathfrak{g})$ on $\mathbf{F}_{u}(s)$.

Next, we consider the pairing $(\bullet, \bullet)$ on $\mathbf{F}_{u}(s)$ defined in [66, sec. 4.3]. Then, let $\mathbf{B}_{u}^{\vee}(s)=\left\{\mathbf{b}_{u}^{\vee}(\mu, s) \mid \mu \in \mathscr{P}^{l}\right\}$ be the $\mathbb{C}(u)$-basis of $\mathbf{F}_{u}(s)$ dual to $\mathbf{B}_{u}^{+}(s)$ with respect to the bilinear form $(\bullet, \bullet)$. It is an upper global basis of $\mathbf{F}_{u}(s)$. Let $\mathbf{B}^{\vee}(s)=$ $\left\{\mathbf{b}^{\vee}(\mu, s) \mid \mu \in \mathscr{P}^{l}\right\}$ be the specialization at $u=1$ of $\mathbf{B}_{u}^{\vee}(s)$, with the obvious labeling of its elements. It is a perfect basis of the usual Fock space $\mathbf{F}(s)=\left.\mathbf{F}_{u}(s)\right|_{u=1}$ by Proposition 1.8.

We equip the set of $l$-partitions with the abstract crystal structure $B(s)=\left(\mathscr{P}^{l}, \widetilde{e}_{i}, \widetilde{f}_{i}\right)$ defined in [44]. Let $B(s)=\left\{b(\mu, s) \mid \mu \in \mathscr{P}^{l}\right\}$ be the obvious labeling. The map $\mathbf{b}^{\vee}(\mu, s) \in \mathbf{B}^{\vee}(s) \longmapsto b(\mu, s) \in B(s)$ is a crystal isomorphism.

\section{UNIPOTENT REPRESENTATIONS}

In this section we record standard results on unipotent representations of finite reductive groups in non-defining characteristic. A good reference is [7].

\subsection{Basics.}

By an $\ell$-modular system we will mean a triple $(K, \mathscr{O}, \mathrm{k})$ where $K$ is a field of characteristic zero, $\mathscr{O}$ is a complete discrete valuation ring with fraction field $K$, and $\mathrm{k}$ is the residue field of $\mathscr{O}$ with $\operatorname{char}(\mathrm{k})=\ell$. When working with representations of a finite group $\Gamma$, we will always assume that $(K, \mathscr{O}, \mathrm{k})$ is a splitting $\ell$-modular system for $\Gamma$, which means that $K$ and $\mathrm{k}$ are splitting fields for all subgroups of $\Gamma$. When $\Gamma$ comes from an algebraic group in characteristic $p$, we will in addition assume that $\ell \neq p$. This case is usually referred to as the non-defining characteristic case.

Let $R$ be any commutative domain (with 1 ) and $\Gamma$ be a finite group. We will assume that $p$ is invertible in $R$ and $\mathscr{O}$. Let $R \Gamma$ denote the group ring of $\Gamma$ over $R$. For any subset $S \subseteq \Gamma$ such that $|S|$ is invertible in $R$, let $e_{S}$ be the element $e_{S}=|S|^{-1} \sum_{g \in S} g$ in $R \Gamma$. When $S$ is a subgroup, $e_{S}$ is an idempotent. If $R$ is not a field, an $R \Gamma$-module which is free as an $R$-module will be called an $R \Gamma$-lattice.

We shall identify isomorphism classes of irreducible representations over $K$ with their character. When $R$ is a field containing $K$, we denote by $R \operatorname{Irr}(K \Gamma)$ or $R \operatorname{Irr}(\Gamma)$ the $R$-module of class functions $\Gamma \rightarrow R$. It is endowed with the canonical scalar 
product $\langle\bullet, \bullet\rangle_{\Gamma}$, for which the set of irreducible characters $\operatorname{Irr}(K \Gamma)$ of $K \Gamma$ is an orthonormal basis.

\subsection{Unipotent $K G$-modules.}

By a rational group we will mean a pair $(\mathbf{G}, F)$ consisting of an algebraic group $\mathbf{G}$ over $\overline{\mathbb{F}}_{q}$ and a Frobenius endomorphism $F$ of $\mathbf{G}$. Assume that $\mathbf{G}$ is a connected reductive group. Fix a parabolic subgroup $\mathbf{P}$ of $\mathbf{G}$ and an $F$-stable Levi complement $\mathbf{L}$ of $\mathbf{P}$. We do not assume $\mathbf{P}$ to be $F$-stable. Write $L=\mathbf{L}^{F}$ and $G=\mathbf{G}^{F}$.

Let $R_{\mathbf{L} \subset \mathbf{P}}^{\mathbf{G}}$ and ${ }^{*} R_{\mathbf{L} \subset \mathbf{P}}^{\mathbf{G}}$ denote respectively the Lusztig induction and restriction maps from $\mathbb{Z} \operatorname{Irr}(K L)$ to $\mathbb{Z} \operatorname{Irr}(K G)$. We will assume that the Mackey formula holds for $R_{\mathbf{L} \subset \mathbf{P}}^{\mathbf{G}}$ and ${ }^{*} R_{\mathbf{L} \subset \mathbf{P}}^{\mathbf{G}}$, which we know for the groups we will focus on later (see [2] for more details). Under this condition, the Lusztig induction and restriction do not depend on the choice of the parabolic subgroup $\mathbf{P}$. We abbreviate $R_{\mathbf{L} \subset \mathbf{P}}^{\mathbf{G}}=R_{\mathbf{L}}^{\mathbf{G}}$ and ${ }^{*} R_{\mathbf{L} \subset \mathbf{P}}^{\mathbf{G}}={ }^{*} R_{\mathbf{L}}^{\mathbf{G}}$

Let $\mathbf{B}$ be an $F$-stable Borel subgroup of $\mathbf{G}$ and $\mathbf{T}$ be an $F$-stable maximal torus of $\mathbf{G}$ with $\mathbf{T} \subset \mathbf{B}$. Let $\mathbf{N}$ be the normalizer of $\mathbf{T}$ in $\mathbf{G}$. Write $B=\mathbf{B}^{F}, T=\mathbf{T}^{F}$ and $N=\mathbf{N}^{F}$. The groups $\mathbf{B}, \mathbf{N}$ form a reductive $B N$-pair of $\mathbf{G}$ with Weyl group $\mathbf{W}=\mathbf{W}_{\mathbf{G}}$ given by $\mathbf{W}=\mathbf{N} / \mathbf{T}$. Since $\mathbf{B}, \mathbf{N}$ are stable by $F$ and $\mathbf{G}$ is connected, the finite groups $B, N$ form a split BN-pair of $G$ whose Weyl group $W=W(T)$ is given by $W=\mathbf{W}^{F}=N / T$.

The $G$-conjugacy classes of $F$-stable maximal tori of $\mathbf{G}$ are parametrized by the $F$ conjugacy classes in $\mathbf{W}$. For each $w \in \mathbf{W}$ let $\mathbf{T}_{w}$ be an $F$-stable maximal torus in the $G$-conjugacy class parametrized by the $F$-conjugacy class of $w$. Under conjugation by some element of $\mathbf{G}$, the pair $\left(\mathbf{T}_{w}, F\right)$ is identified with the pair $(\mathbf{T}, w F)$. In particular, we have $T_{w} \simeq \mathbf{T}^{w F}$ and $W\left(T_{w}\right) \simeq \mathbf{W}^{w F}$. The virtual characters $R_{\mathbf{T}_{w}}^{\mathbf{G}}(1)$ obtained by induction of the trivial representation of the tori $T_{w}$ are called the virtual Deligne-Lusztig characters.

Definition 3.1. An irreducible $K G$-module is unipotent if its character, say $\chi$, occurs as a constituent of a virtual Deligne-Lusztig character $R_{\mathbf{T}_{w}}^{\mathbf{G}}(1)$ for some element $w \in W$, i.e., if we have $\left\langle\chi, R_{\mathbf{T}_{w}}^{\mathbf{G}}(1)\right\rangle_{G} \neq 0$.

We denote by $K G$-umod the full subcategory of $K G$-mod consisting of the modules which are sums of irreducible unipotent modules. The objects of this category are the unipotent KG-modules.

Remark 3.2. Fix an element $\sigma$ in the normalizer of $\mathbf{L}$ in $\mathbf{G}$ such that the conjugation by $\sigma$ yields an element in the normalizer of $\mathbf{W}_{\mathbf{L}}$ in $\mathbf{W}$. Let us denote it again by the symbol $\sigma$. Then, we can equip the subgroup $\mathbf{L} \subseteq \mathbf{G}$ with the rational structure with the Frobenius endomorphism $\sigma F$ and we have Lusztig induction and restriction 
maps $R_{\mathbf{L}, \sigma F}^{\mathbf{G}, F}$ and ${ }^{*} R_{\mathbf{L}, \sigma F}^{\mathbf{G}, F}$ between $\mathbb{Z} \operatorname{Irr}\left(K \mathbf{L}^{\sigma F}\right)$ and $\mathbb{Z} \operatorname{Irr}(K G)$. In the particular case $\sigma=1$ considered above we have $R_{\mathbf{L}, F}^{\mathbf{G}, F}=R_{\mathbf{L}}^{\mathbf{G}}$ and ${ }^{*} R_{\mathbf{L}, F}^{\mathbf{G}, F}={ }^{*} R_{\mathbf{L}}^{\mathbf{G}}$. If $\mathbf{T}_{w}$ is as above then we have also $R_{\mathbf{T}, w F}^{\mathbf{G}, F}=R_{\mathbf{T}_{w}}^{\mathbf{G}}$ and ${ }^{*} R_{\mathbf{T}, w F}^{\mathbf{G}, F}={ }^{*} R_{\mathbf{T}_{w}}^{\mathbf{G}}$.

\subsection{Unipotent $\mathrm{k} G$-modules and $\ell$-blocks.}

As a result of the lifting of idempotents, the blocks of $\mathscr{O} G$ and $\mathrm{k} G$ correspond by reduction. Both are usually called the $\ell$-blocks of $G$. For $R=\mathscr{O}$ or $\mathrm{k}$, any block $B$ of $R G$ is of the form $B=R G \cdot b$, where $b$ is a central primitive idempotent of $R G$. The unit $b$ of $B$ is called the block idempotent of $B$. We will also call block of $R G$-mod associated with $B$ the Serre subcategory generated by the simple modules on which $b$ acts non-trivially. The $\ell$-blocks of $G$ induce a partition of $\operatorname{Irr}(K G)$ such that the piece associated with $B$ is the set of all irreducible characters $\chi$ of $K G$ with $\chi(b)=\chi(1)$. If $\chi \in \operatorname{Irr}(K G)$, we will write $B(\chi) \subseteq \operatorname{Irr}(K G)$ for the piece containing $\chi$. When there is no risk of confusion, we will also call $B(\chi)$ an $\ell$-block of $G$.

Definition 3.3. An $\ell$-block of $\mathscr{O} G$ is unipotent if it contains at least one unipotent $K G$-module. A simple $\mathrm{k} G$-module is unipotent if it lies in a unipotent block of $\mathrm{k} G$.

We denote by $\mathrm{k} G$-umod the Serre subcategory of $\mathrm{k} G$-mod generated by the simple unipotent $\mathrm{k} G$-modules. It corresponds to the sum of unipotent blocks of $\mathrm{k} G$-mod. The unipotent $\mathrm{k} G$-modules are by definition the objects of this category.

Recall that $(K, \mathscr{O}, \mathrm{k})$ is a splitting $\ell$-modular system for $G$. To this system one can associate a decomposition map $d_{\mathscr{O} G}:[K G-\mathrm{mod}] \rightarrow[\mathrm{k} G$-mod]. By [40, thm. 3.1], a simple $\mathrm{k} G$-module is unipotent if and only if it is a constituent of the $\ell$-reduction of a unipotent $K G$-module, see also [5]. In other words, the classes of unipotent modules are exactly the image of unipotent characters through the decomposition map. We will denote by $d_{\mathscr{U}}:[K G$-umod $] \rightarrow[\mathrm{k} G$-umod $]$ the restriction of this map to unipotent characters.

Theorem 3.4 (Geck-Hiss [29], Geck [27]). Assume $\ell$ is good for $\mathbf{G}$ and that $\ell \nmid$ $\left|Z(\mathbf{G}) / Z(\mathbf{G})^{\circ}\right|$. Then the map $d_{\mathscr{U}}$ is a linear isomorphism $[K G$-umod $] \stackrel{\sim}{\rightarrow}[\mathrm{k} G$-umod] .

Note that if $\mathbf{G}$ is semisimple with no component of type $A$, then the condition $\ell \nmid\left|Z(\mathbf{G}) / Z(\mathbf{G})^{\circ}\right|$ is superfluous since every prime divisor of $\left|Z(\mathbf{G}) / Z(\mathbf{G})^{\circ}\right|$ is a bad prime.

Given a positive integer $f$, let $\Phi_{f}$ be the $f$ th cyclotomic polynomial. If $\mathbf{T} \subset \mathbf{G}$ is an $F$-stable torus, then the order of $T:=\mathbf{T}^{F}$ is a polynomial in $q$ with coefficients in $\mathbb{Z}$. We say that $\mathbf{T}$ (and by extension $T$ ) is a $\Phi_{f}$-torus if this polynomial is a power of $\Phi_{f}$. An $F$-stable Levi subgroup $\mathbf{L} \subseteq \mathbf{G}$ is $f$-split if it is the centralizer in $\mathbf{G}$ of 
a $\Phi_{f}$-torus. A unipotent $f$-pair is a pair $(\mathbf{L}, \chi)$ where $\mathbf{L}$ is an $f$-split Levi subgroup and $\chi$ is an irreducible unipotent $K L$-module. The pair $(\mathbf{L}, \chi)$ is $f$-cuspidal if for every proper $f$-split Levi subgroup $\mathbf{M} \subseteq \mathbf{L}$ we have ${ }^{*} R_{\mathbf{M}}^{\mathbf{L}}(\chi)=0$.

Now assume that $f$ is the smallest positive integer such that $\ell$ divides $q^{f}-1$. Under the assumption that $\ell$ is good, unipotent $\ell$-blocks correspond to $f$-cuspidal $f$-pairs.

Theorem 3.5 (Broué-Malle-Michel [4], Cabanes-Enguehard [7]). Assume $\ell$ is good for $\mathbf{G}$, and $\ell \neq 3$ if $\mathbf{G}$ has a component of type ${ }^{3} D_{4}$. Then there is a bijection between the $G$-conjugacy classes of unipotent $f$-cuspidal $f$-pairs and the set of unipotent $\ell$ blocks of $G$ which takes the class of $(\mathbf{L}, \chi)$ to the $\ell$-block $B_{\mathbf{L}, \chi}$ such that the irreducible unipotent characters in $B_{\mathbf{L}, \chi}$ are exactly the irreducible constituents of $R_{\mathbf{L}}^{\mathbf{G}}(\chi)$.

\subsection{Harish-Chandra series.}

Assume now that the parabolic subgroup $\mathbf{P} \subseteq \mathbf{G}$ considered in $₫ 3.2$ is $F$-stable. In that case the group $L$ is $G$-conjugate to a standard Levi subgroup of $G$. Let $R_{L}^{G}$ and ${ }^{*} R_{L}^{G}$ be the corresponding Harish-Chandra induction and restriction functors between $R L$-mod and $R G$-mod. Let $P=\mathbf{P}^{F}$ and $U=\mathbf{U}^{F}$, where $\mathbf{U} \subset \mathbf{P}$ is the unipotent radical of $\mathbf{P}$. Notice that the Harish-Chandra induction is the special case of Lusztig induction for 1-split Levi subgroups.

The order of $U$ is a power of $q$, hence it is invertible in $R$. Thus, for all $M \in$ $R L$-mod, $N \in R G$-mod we have

$$
R_{L}^{G}(M)=R G \cdot e_{U} \otimes_{R L} M \text { and }{ }^{*} R_{L}^{G}(N)=e_{U} \cdot R G \otimes_{R G} N .
$$

We will say that the functors $R_{L}^{G}$ and ${ }^{*} R_{L}^{G}$ are represented by the $(R G, R L)$-bimodule $R G \cdot e_{U}$ and the $(R L, R G)$-bimodule $e_{U} \cdot R G$ respectively.

Here are some well-known basic properties of the functors $R_{L}^{G},{ }^{*} R_{L}^{G}$.

(a) $R_{L}^{G},{ }^{*} R_{L}^{G}$ do not depend on $P$,

(b) $R_{L}^{G},{ }^{*} R_{L}^{G}$ are exact and left and right adjoint to one another,

(c) if $L \subseteq M \subseteq G$ are Levi subgroups there are isomorphisms of functors $R_{L}^{G}=$ $R_{M}^{G} R_{L}^{M}$ and ${ }^{*} R_{L}^{G}={ }^{*} R_{L}^{M *} R_{M}^{G}$

Let $R=K$ or $\mathrm{k}$. An irreducible $R G$-module $E$ is cuspidal if ${ }^{*} R_{L}^{G}(E)=0$ for all proper standard Levi subgroups $L \subsetneq G$. A cuspidal pair of $R G$ is a pair $(L, E)$ where $L$ is as above and $E \in \operatorname{Irr}(R L)$ is cuspidal. Since the group $L$ is uniquely recovered from $E$, from now on we may omit it from the notation. Then, the set $\operatorname{Irr}(R G, E) \subseteq \operatorname{Irr}(R G)$ consisting of the constituents of the head of $R_{L}^{G}(E)$ is equal to the set of constituents of the socle of $R_{L}^{G}(E)$ and is called the Harish-Chandra 
series of $(L, E)$. The $R$-algebra $\mathscr{H}(R G, E)=\operatorname{End}_{R G}\left(R_{L}^{G}(E)\right)^{\text {op }}$ is its ramified Hecke algebra. We have the following facts:

(d) the Harish-Chandra series form a partition of $\operatorname{Irr}(R G)$,

(e) the functor $\mathfrak{F}_{R_{L}^{G}(E)}$ as defined in $\$ 1.1$ yields a bijection

$$
\operatorname{Irr}(R G, E) \stackrel{1: 1}{\longleftrightarrow} \operatorname{Irr}(\mathscr{H}(R G, E)) .
$$

Proposition 3.6 ([3]). If $R=K$ or $\mathrm{k}$, then the Harish-Chandra induction and restriction functors preserve the category of unipotent $R G$-modules.

\section{Finite Unitary Groups}

This section is devoted to the construction of categorical actions on the category of unipotent representations of finite unitary groups $\mathrm{GU}_{n}(q)$.

Let $R$ be a commutative domain with unit. Under mild assumptions on $R$, we construct in 4.2 a representation datum on the abelian category

$$
R G-\bmod :=\bigoplus_{n \geqslant 0} \mathrm{GU}_{n}(q)-\bmod
$$

given by Harish-Chandra induction and restriction. When $R$ is an extension of $\mathbb{Q}_{\ell}$ or $\mathbb{F}_{\ell}$, the categorical datum restricts to the category $\mathscr{U}_{R}$ of unipotent representations of $R G$-mod. On this smaller category, the eigenvalues of $X$ are powers of $-q$, which hints that the Lie algebra $\mathfrak{g}$ that should act on $\left[\mathscr{U}_{R}\right]$ corresponds to the quiver with vertices $(-q)^{\mathbb{Z}}$ and arrows given by multiplication by $q^{2}$.

We prove that the representation datum lifts indeed to a categorical action of $\mathfrak{g}$ on $\mathscr{U}_{R}$. The situation depends on the parity of $e$, the order of $-q$ modulo $\ell$. When $e$ is even, i.e., $\ell$ is a linear prime, $\mathfrak{g}$ is a subalgebra of $\left(\widehat{\mathfrak{s l}_{e / 2}}\right)^{\oplus 2}$ and each ordinary Harish-Chandra series categorifies a level 2 Fock space for $\mathfrak{g}$. When $e$ is odd (unitary prime case), $\mathfrak{g} \simeq \widehat{\mathfrak{s l}}_{\mathrm{e}}$ and weight spaces correspond to unipotent $\ell$-blocks, which are now transverse to the ordinary Harish-Chandra series.

For studying the weight space decomposition of $\left[\mathscr{U}_{R}\right]$ as well as the action of the affine Weyl group we use the action of a bigger Lie algebra $\mathfrak{g}_{\circ}$, which comes from Harish-Chandra induction and restriction for general linear groups. Going from linear to unitary groups by Ennola duality introduces signs for this action (see Lemma 4.15). It is to be expected that the action of $\mathfrak{g}_{\circ}$ on $\left[\mathscr{U}_{R}\right]$ lifts to an action by triangulated functors on $D^{b}\left(\mathscr{U}_{R}\right)$ coming from Lusztig induction, although we will not use it. See [19] for more details. 


\subsection{Definition.}

Fix a positive integer $n$. We equip the reductive algebraic group $\mathbf{G L}_{n}=\mathrm{GL}_{n}\left(\overline{\mathbb{F}}_{q}\right)$ with the standard Frobenius map $F_{q}: \mathbf{G L}_{n} \rightarrow \mathbf{G L}_{n},\left(a_{i j}\right) \mapsto\left(a_{i j}^{q}\right)$ given by raising every coefficient to the $q$ th power. The finite general linear group $\mathrm{GL}_{\mathrm{n}}(q)$ is given by the fixed points of $\mathbf{G L}_{n}$ under $F_{q}$. In this section we will work with a twisted version of this group obtained by twisting the Frobenius map. Let $J_{n}$ be the $n \times n$ matrix with entry 1 in $(i, n-i+1)$ and zero elsewhere. We will often write $J=J_{n}$ when there is no risk of confusion on the size of the matrices. We define a new Frobenius map $F$ on $\mathbf{G L}_{n}$, called the twisted Frobenius map, by setting $F=F_{q} \circ \alpha$ where $\alpha(g)=J \cdot{ }^{t} g^{-1} \cdot J$ for each $g \in \mathbf{G L}_{n}$. The finite unitary group $G_{n}=\mathrm{GU}_{n}(q)$ is then given by

$$
G_{n}=\left(\mathbf{G L}_{n}\right)^{F}=\left\{g \in \mathbf{G L}_{n} ; F(g)=g\right\}
$$

Since $F^{2}=F_{q^{2}}$ we have $G_{n} \subset G L_{n}$, where we abbreviate

$$
G L_{n}=\mathbf{G L}_{n}\left(q^{2}\right):=\left(\mathbf{G L}_{n}\right)^{F^{2}} .
$$

By convention we also define $G_{0}=\{1\}$ to be the trivial group.

We equip $\mathbf{G L}_{n}$ with the standard split BN-pair such that $\mathbf{B}$ is the subgroup of upper triangular matrices and $\mathbf{N}$ is the subgroup of all monomial matrices. Since $\mathbf{B}$, $\mathbf{N}$ are stable by $F$ and $\mathbf{G L}_{n}$ is connected, the groups $B=\mathbf{B}^{F}, N=\mathbf{N}^{F}$ form a split BN-pair of the finite group $G_{n}$. Let $\mathbf{T}$ be the diagonal torus in $\mathbf{G L}_{n}$ and $T=\mathbf{T}^{F}$. Let $\mathbf{W}=\mathbf{W}_{n}$ be the Weyl group of $\mathbf{G L}_{n}$ and $W=W_{n}$ be the Weyl group of $G_{n}$. We have $\mathbf{W} \simeq \mathfrak{S}_{n}$, and $F$ induces on $\mathbf{W}$ the automorphism given by conjugation with the longest element $w_{0}$. We will embed $\mathbf{W}$ in $G_{n}$ using permutation matrices, so that $w_{0}$ corresponds to $J$. We have $W=\mathbf{W}^{F}=C_{\mathbf{W}}\left(w_{0}\right)$. It is a Weyl group of type $B_{m}$ if $n=2 m$ or $2 m+1$.

Let $\varepsilon_{1}, \ldots, \varepsilon_{n}$ be the characters of $\mathbf{T}$ such that $t=\operatorname{diag}\left(\varepsilon_{1}(t), \varepsilon_{2}(t), \ldots, \varepsilon_{n}(t)\right)$ for all $t \in \mathbf{T}$. The roots (resp. simple roots) of $\mathbf{G L}_{n}$ are given by $\left\{\varepsilon_{i}-\varepsilon_{j} \mid i \neq j\right\}$ (resp. $\left.\left\{\varepsilon_{r}-\varepsilon_{r+1}\right\}\right)$. Let $s_{r}=(r, r+1)$ be the simple reflection in $\mathbf{W}$ associated with the simple root $\alpha_{r}=\varepsilon_{r}-\varepsilon_{r+1}$. The action of $F$ on the roots induces an automorphism $\sigma$ of the Dynkin diagram of $\mathbf{G L}_{n}$ such that

$$
F \circ \alpha_{r}^{\vee}=q \sigma\left(\alpha_{r}\right)^{\vee} \text { with } \sigma\left(\alpha_{r}\right)=\alpha_{n-r} .
$$

For each root $\alpha$, let $\mathbf{U}_{\alpha}$ and $\alpha^{\vee} \in \operatorname{Hom}\left(\mathbb{G}_{m}, \mathbf{T}\right)$ be the corresponding root subgroup and cocharacter. We also choose an isomorphism $u_{\alpha}: \mathbb{G}_{a} \stackrel{\sim}{\rightarrow} \mathbf{U}_{\alpha}$ such that $F\left(u_{\sigma(\alpha)}(t)\right)=u_{\alpha}\left(t^{q}\right)$. Note that a one-parameter subgroup of $T$ has either $\left(q^{2}-1\right)$ or $(q+1)$ elements, and a root subgroup of $G_{n}$ has either $q$ or $q^{2}$ elements.

The standard Levi subgroups $L_{r, \lambda}$ of $G_{n}$ are parametrized by pairs $(r, \lambda)$ where $r$ is a non-negative integer and $\lambda=\left(\lambda_{1}, \lambda_{2}, \ldots, \lambda_{s}\right)$ is a tuple of positive integers such 
that $n=r+2 \sum_{u=1}^{s} \lambda_{u}$. The group $L_{r, \lambda}$ consists of all matrices of $\mathbf{G L}_{n}$ which belong to $G_{n}$ and are of block-diagonal type

$$
\prod_{u=1}^{s} \mathbf{G L}_{\lambda_{u}} \times \mathbf{G L}_{r} \times \prod_{u=s}^{1} \mathbf{G L}_{\lambda_{u}} .
$$

Consequently we have a group isomorphism $L_{r, \lambda} \simeq G_{r} \times \prod_{u} G L_{\lambda_{u}}$. If $m$ is a positive integer we abbreviate $L_{r, 1^{m}}=L_{r,\left(1^{m}\right)}$ and $L_{r, m}=L_{r,(m)}$.

\subsection{The representation datum on $R G$-mod.}

Let $R$ be a commutative domain with unit. We assume that $q\left(q^{2}-1\right)$ in invertible in $R$. Using parabolic induction and restriction, we show in this section how to construct a representation datum on

$$
R G-\bmod :=\bigoplus_{n \in \mathbb{N}} R G_{n}-\bmod .
$$

Fix a positive integer $n$. Parabolic (or Harish-Chandra) induction provides functors between $L$-mod and $G_{n}$-mod for any standard Levi subgroup $L=L_{r, m} \subset G_{n}$. Since we want functors between $G_{r}$-mod and $G_{n}$-mod we will consider a slight variation of the usual parabolic induction.

Let $0 \leqslant r<n$. We denote by $V_{r}$ the unipotent radical of the standard parabolic subgroup $P_{r, 1} \subset G_{r+2}$ with Levi complement $L_{r, 1}$. Let $U_{r} \subset G_{r+2}$ be the subgroup given by

$$
U_{r}=V_{r} \rtimes \mathbb{F}_{q^{2}}^{\times}=\left(\begin{array}{ccccc}
* & * & \cdots & \cdots & * \\
& 1 & & (0) & \vdots \\
& & \ddots & & \vdots \\
& (0) & & 1 & * \\
& & & & *
\end{array}\right)
$$

so that $P_{r, 1}=V_{r} \rtimes L_{r, 1} \simeq U_{r} \rtimes G_{r}$. If $n-r$ is even, we set $U_{n, r}=U_{n-2} \rtimes \cdots \rtimes U_{r}$ and we define $e_{n, r}=e_{U_{n, r}}$ to be the corresponding idempotent. In particular, we have $e_{r+2, r}=e_{U_{r}}$. We embed $G_{r}$ into the Levi subgroup $L_{r, 1^{m}}=G_{r} \times G L_{1}^{m}$ in the obvious way. This yields an embedding $G_{r} \subset G_{n}$ and functors

$$
\begin{aligned}
& F_{n, r}=R G_{n} \cdot e_{n, r} \otimes_{R G_{r}} \bullet: R G_{r}-\bmod \longrightarrow R G_{n}-\bmod , \\
& E_{r, n}=e_{n, r} \cdot R G_{n} \otimes_{R G_{n}} \bullet: R G_{n}-\bmod \longrightarrow R G_{r}-\bmod .
\end{aligned}
$$

Note that $F_{n, r}$ can be seen as the composition of the inflation $G_{r}-\bmod \longrightarrow L_{r, 1^{m}}-\bmod$ with the parabolic induction from $L_{r, 1^{m}}$ to $G_{n}$. 
An endomorphism of the functor $F_{n, r}$ can be represented by an $\left(R G_{n}, R G_{r}\right)$ bimodule endomorphism of $R G_{n} \cdot e_{n, r}$, or equivalently by an element of $e_{n, r} \cdot R G_{n} \cdot e_{n, r}$ centralizing $R G_{r}$. Thus, the elements

$$
X_{r+2, r}=(-q)^{r} e_{r+2, r}(1, r+2) e_{r+2, r}, \quad T_{r+4, r}=q^{2} e_{r+4, r}(1,2)(r+3, r+4) e_{r+4, r}
$$

define respectively natural transformations of the functors $F_{r+2, r}$ and $F_{r+4, r}$. We set

$$
F=\bigoplus_{r \geqslant 0} F_{r+2, r}, \quad X=\bigoplus_{r \geqslant 0} X_{r+2, r}, \quad T=\bigoplus_{r \geqslant 0} T_{r+4, r} .
$$

Proposition 4.1. The tuple $(E, F, X, T)$ defines a representation datum with parameter $q^{2}$ on $R G-\bmod =\bigoplus_{n \in \mathbb{N}} R G_{n}-\bmod$.

Remark 4.2. The reader might argue that we did not check that $X$ was invertible. Nevertheless, we shall only be working with unipotent modules over a field, in which case the eigenvalues of $X$ are powers of $-q$, hence non-zero (see Theorem 4.8). This will ensure that the restriction of $X$ to this category is indeed invertible.

\subsection{The categories of unipotent modules $\mathscr{U}_{K}$ and $\mathscr{U}_{\mathrm{k}}$.}

From now on, we fix a prime number $\ell$ such that $\ell \nmid q\left(q^{2}-1\right)$, and an $\ell$-modular system $(K, \mathscr{O}, \mathrm{k})$ with $\mathbb{Q}_{\ell} \subset K$ and $\overline{\mathbb{F}}_{\ell} \subset \mathrm{k}$. Although this modular system is not large enough for all the finite groups encountered, it will be enough for our purpose since we will be working with unipotent characters only, which are defined over $\mathbb{Q}_{\ell}$ (see below).

Throughout the following sections, we will denote by $d, e$ and $f$ the order of $q^{2}$, $-q$ and $q$ in $\mathrm{k}^{\times}$. In particular $e \neq 1,2$. If $e$ is odd, then $d=e$ and $f=2 e$; if $e$ is even, then $d=e / 2$ and either $f=e / 2$ if $e \equiv 2 \bmod 4$ or $f=e$ if $e \equiv 0 \bmod 4$.

4.3.1. The category $\mathscr{U}_{K}$. Fix a positive integer $n$. By [56], the irreducible unipotent $K G_{n}$-modules are labelled by partitions of $n$. Their character can be directly constructed from the virtual Deligne-Lusztig characters. Namely, for each $w \in \mathfrak{S}_{n}$, fix an $F$-stable maximal torus $\mathbf{T}_{w} \subseteq \mathbf{G L}_{n}$ in the $G_{n}$-conjugacy class parametrized by the $\mathfrak{S}_{n}$-conjugacy class of $w F$ in the coset $\mathfrak{S}_{n} F$, with the convention that $\mathbf{T}_{1}=\mathbf{T}$. Then the class function

$$
\chi_{\lambda}=\left|\mathfrak{S}_{n}\right|^{-1} \sum_{w \in \mathfrak{S}_{n}} \phi_{\lambda}\left(w w_{0}\right) R_{\mathbf{T}_{w}}^{\mathbf{G L}_{n}}(1) \in \mathbb{Z} \operatorname{Irr}\left(K G_{n}\right)
$$

is, up to a sign, an irreducible unipotent character. Since the cohomology groups are defined over $\mathbb{Q}_{\ell} \subset K$ and since any character of $\mathfrak{S}_{n}$ has rational values, either $\chi_{\lambda}$ or $-\chi_{\lambda}$ is the character of some irreducible $K G_{n}$-module which we denote by $E_{\lambda}$. By 
abuse of notation we will still denote by $E_{\lambda}$ its isomorphism class. Let $\varepsilon_{\lambda}$ be the sign determined by the relation $\chi_{\lambda}=\varepsilon_{\lambda} E_{\lambda}$.

Recall that $G_{0}=\{1\}$. We call the category of unipotent $K G$-modules the category

$$
\mathscr{U}_{K}:=\bigoplus_{n \in \mathbb{N}} K G_{n}-\text { umod }
$$

This category is abelian semisimple. From the previous discussion we have

$$
\operatorname{Irr}\left(\mathscr{U}_{K}\right)=\left\{E_{\lambda} \mid \lambda \in \mathscr{P}\right\}
$$

where by convention $\operatorname{Irr}\left(K G_{0}\right)=\left\{E_{\emptyset}\right\}$.

4.3.2. The category $\mathscr{U}_{\mathrm{k}}$. Using the $\ell$-modular system we have decomposition maps $d_{\mathscr{O} G_{n}}$ which by Proposition 3.4 restrict to linear isomorphisms

$$
d_{\mathscr{O} G_{n}}:\left[K G_{n} \text {-umod }\right] \stackrel{\sim}{\rightarrow}\left[\mathrm{k} G_{n} \text {-umod }\right] .
$$

Note that we do not need to assume that $K G_{n}$ is a split algebra since we are only interested in unipotent $K G_{n}$-modules, which are all absolutely irreducible.

For unitary groups, this map is actually unitriangular with respect to the basis of irreducible modules and the dominance order.

Theorem 4.3 ([26]). There is a unique labelling $\left\{D_{\lambda}\right\}_{\lambda \in \mathscr{P}_{n}}$ of the unipotent simple $\mathrm{k} G_{n}$-modules such that $d_{\mathscr{O} G_{n}}\left(\left[E_{\lambda}\right]\right) \in\left[D_{\lambda}\right]+\sum_{\nu>\lambda} \mathbb{Z}\left[D_{\nu}\right]$ where $>$ is the dominance order on partitions of $n$.

We call the category of unipotent $\mathrm{k} G$-modules the category

$$
\mathscr{U}_{\mathrm{k}}:=\bigoplus_{n \in \mathbb{N}} \mathrm{k} G_{n}-\text { umod }
$$

This is an abelian category which is not semisimple. The isomorphism classes of simple objects are $\operatorname{Irr}\left(\mathscr{U}_{\mathrm{k}}\right)=\left\{D_{\lambda}\right\}_{\lambda \in \mathscr{P}}$ and the decomposition map yields a $\mathbb{Z}$-linear isomorphism $d_{\mathscr{U}}:\left[\mathscr{U}_{K}\right] \stackrel{\sim}{\rightarrow}\left[\mathscr{U}_{\mathrm{k}}\right]$. The following result is a consequence of the unitriangularity of this map.

Proposition 4.4. Given a partition $\lambda \vdash n$ there is a unique $\mathscr{O} G_{n}$-lattice $\widetilde{E}_{\lambda}$ such that

(a) $K \widetilde{E}_{\lambda}=E_{\lambda}$ as a $K G_{n}$-module,

(b) $V_{\lambda}:=\mathrm{k} \widetilde{E}_{\lambda}$ is an indecomposable $\mathrm{k} G_{n}$-module with head isomorphic to $D_{\lambda}$,

(c) $d_{\mathscr{U}}\left(\left[E_{\lambda}\right]\right)=\left[V_{\lambda}\right]$. 
4.3.3. Blocks of $\mathscr{U}_{\mathrm{k}}$. A block of $\mathscr{U}_{\mathrm{k}}$ is an indecomposable summand of $\mathscr{U}_{\mathrm{k}}$. Therefore blocks of $\mathscr{U}_{\mathrm{k}}$ correspond to the unipotent blocks of $\mathrm{k} G_{n}$ where $n$ runs over $\mathbb{N}$. Recall that $e$ is the order of $-q$ modulo $\ell$.

Proposition $4.5([22])$. The map $E_{\lambda} \longmapsto\left(\lambda_{[e]}, w_{e}(\lambda)\right)$ yields a bijection between unipotent $\ell$-blocks and pairs $(s, w)$ where $s \in \mathbb{Z}^{e}(0)$ and $w \in \mathbb{N}$.

Recall that $\nu \mapsto \nu_{[e]}$ induces a bijection between $e$-cores and $\mathbb{Z}^{e}(0)$. Given $\nu$ an $e$-core we will denote by $B_{\nu, w}$ or $B_{\nu_{[e]}, w}$ the unipotent $\ell$-block containing all the unipotent characters $E_{\lambda}$ such that $\nu$ is the $e$-core of $\lambda$ and $w_{e}(\lambda)=w$. It is a block of $\mathrm{k} G_{n}$ with $n=|\nu|+e w$.

It also follows from the classification of blocks that when $w<e$ and $e \neq 1$, the defect group of $B_{\nu, w}$ is an elementary abelian $\ell$-group of rank $w$ (see 4]). In particular, when $w=0$ the module $D_{\lambda}$ is simple and projective, isomorphic to $V_{\lambda}$, and when $w=1$ the defect group of $B_{\nu, w}$ is a cyclic group. The structure of such blocks was determined in [24].

4.3.4. The weak Harish-Chandra series. For this section we assume that $R$ is one of the fields $K$ or $\mathrm{k}$.

Let $r, m \geqslant 0$ and $n=r+2 m$. The inflation from $G_{r}$ to $L_{r, 1^{m}}$ yields an equivalence between $R G_{r}$-umod and $R L_{r, 1^{m}}$-umod, since the Deligne-Lusztig varieties depend only on the semisimple type of the reductive group. This equivalence intertwines the functors $E_{n, r}, F_{n, r}$ with the parabolic restriction and induction ${ }^{*} R_{L_{r, 1 m}}^{G_{n}}$, $R_{L_{r, 1} m}^{G_{n}}$. Therefore working with $\mathscr{U}_{R}$ and the functors $E$ and $F$ is the same as working in the usual framework of unipotent representations and Harish-Chandra induction/restriction from Levi subgroups. Note however that we do not consider all the standard Levi subgroups, but only the ones that are conjugate to $L_{r, 1^{m}}$. Therefore we need to consider a slight variation of the usual Harish-Chandra theory. The following definitions and properties are taken from [36.

Definition 4.6. Fix a non-negative integer $n$.

(a) An $R G_{n}$-module $D$ is weakly cuspidal if ${ }^{*} R_{L}^{G_{n}}(D)=0$ for any Levi subgroup $L \subsetneq G_{n}$ which is $G_{n^{-}}$-conjugate to a subgroup of the form $L_{r, 1^{m}}$.

(b) A weakly cuspidal pair of $R G_{n}$ is a pair which is $G_{n^{-}}$-conjugate to $\left(L_{r, 1^{m}}, D\right)$ for some $n=r+2 m$ and some weakly cuspidal irreducible $R G_{r}$-module $D$ which is viewed as a $\mathrm{k} L_{r, 1^{m}}$-module by inflation.

(c) The weak Harish-Chandra series $\operatorname{WIrr}\left(R G_{n}, D\right)$ of $R G_{n}$ determined by the weakly cuspidal pair $(L, D)$ is the set of the constituents of the head of $R_{L}^{G_{n}}(D)$. By [36], it coincides with the set of the constituents of its socle. 
If $D$ is a unipotent $R G_{r}$-module, then $D$ is weakly cuspidal if and only if $E(D)=0$. Moreover, if $D$ is irreducible, the weak Harish-Chandra series coincides with the set of irreducible constituents in the head of $F^{m} D$. Therefore it makes sense to define the weak Harish-Chandra series of $D$ in $\mathscr{U}_{R}$ by

$$
\operatorname{WIrr}(R G, D)=\bigsqcup_{n \geqslant r} \operatorname{WIrr}\left(R G_{n}, D\right)
$$

It is the set of irreducible consituents in the head of $F^{k} D$ for some $k \geqslant 0$ (or equivalently in the socle). As in the case of the usual theory, the weak Harish-Chandra series of $\mathscr{U}_{R}$ form a partition of $\operatorname{Irr}\left(\mathscr{U}_{R}\right)$, i.e., we have $\operatorname{Irr}\left(\mathscr{U}_{R}\right)=\bigsqcup \mathrm{W} \operatorname{Irr}(R G, D)$ where the sum runs over the set of isomorphism classes of weakly cuspidal unipotent irreducible modules $D$.

When $R=K$, weakly cuspidal unipotent modules coincide with cuspidal modules and were determined in [53]. One of the main results in this paper is the classification of the weakly cuspidal modules in the case where $R=\mathrm{k}$.

\subsection{The $\mathfrak{g}_{\infty}$-representation on $\mathscr{U}_{K}$.}

In this section we show how the categorical datum defined in $\$ 4.2$ yields a categorical representation on $\mathscr{U}_{R}$ in the case where $R=K$. This is achieved by translating into our framework the theory of Lusztig and Howlett-Lehrer on endomorphism algebras of representations induced from a cuspidal module in [53] and [42].

4.4.1. Action of $E$ and $F$. Since every unipotent character is a linear combination of (virtual) Deligne-Lusztig characters - we call such a class function a uniform function - it is well-known how to compute the action of $E$ and $F$ on the category $\mathscr{U}_{K}$.

Lemma 4.7. Let $\lambda$ be a partition. Then $[E]\left(\left[E_{\lambda}\right]\right)=\sum_{\mu}\left[E_{\mu}\right]$ and $[F]\left(\left[E_{\lambda}\right]\right)=$ $\sum_{\mu^{\prime}}\left[E_{\mu^{\prime}}\right]$ where $\mu$ (resp. $\left.\mu^{\prime}\right)$ runs over the partitions that are obtained from $\lambda$ by removing (resp. adding) a 2-hook.

We deduce that a unipotent $K G_{n}$ module $E_{\lambda}$ is weakly cuspidal if and only if there exists $t \geqslant 0$ such that $n=t(t+1) / 2$ and $\lambda=\Delta_{t}=(t, t-1, \ldots, 1)$. We will set $E_{t}:=E_{\Delta_{t}}$. Consequently, the weakly cuspidal pairs are, up to conjugation, the pairs $\left(L_{r, 1^{m}}, E_{t}\right)$ with $r=t(t+1) / 2$. The partition into series is thus $\operatorname{Irr}\left(\mathscr{U}_{K}\right)=$ $\bigsqcup_{t \in \mathbb{N}} \operatorname{WIrr}\left(K G, E_{t}\right)$. 
4.4.2. The ramified Hecke algebra. Let $r, m$ be non-negative integers and $n=r+2 m$. As mentioned in 4 4.3.4 the inflation from $G_{r}$ to $L_{r, 1^{m}}$ yields an equivalence between $K G_{r}$-umod and $K L_{r, 1^{m}}$-umod which intertwines the functor $F_{n, r}$ with the parabolic induction $R_{L_{r, 1} m}^{G_{n}}$. In particular, we have a canonical isomorphism

$$
\mathscr{H}\left(K G_{n}, E_{t}\right):=\operatorname{End}_{K G_{n}}\left(F^{m}\left(E_{t}\right)\right)^{\text {op }} \stackrel{\sim}{\rightarrow} \operatorname{End}_{K G_{n}}\left(R_{L_{r, 1} m}^{G_{n}}\left(E_{t}\right)\right)^{\text {op }} .
$$

Now recall from $\$ 1.3 .1$ that to the categorical datum $(E, F, X, T)$ is attached a map $\phi_{F^{m}}: \mathbf{H}_{K, m}^{q^{2}} \rightarrow \operatorname{End}\left(F^{m}\right)$. The evaluation of this map at the module $E_{t}$ yields a $K$-algebra homomorphism

$$
\phi_{K, m}: \mathbf{H}_{K, m}^{q^{2}} \rightarrow \mathscr{H}\left(K G_{n}, E_{t}\right), \quad X_{k} \mapsto X_{k}\left(E_{t}\right), \quad T_{l} \mapsto T_{l}\left(E_{t}\right) .
$$

By [42], $\mathscr{H}\left(K G_{n}, E_{t}\right)$ is isomorphic to a Hecke algebra $\mathbf{H}_{K, m}^{Q_{t} ; q^{2}}$ of type $B_{m}$ with

$$
Q_{t}= \begin{cases}\left((-q)^{-1-t},(-q)^{t}\right) & \text { if } t \text { is even } \\ \left((-q)^{t},(-q)^{-1-t}\right) & \text { if } t \text { is odd }\end{cases}
$$

The previous map provides indeed such an isomorphism.

Theorem 4.8. Let $t, m \geqslant 0$ and $n=t(t+1) / 2+2 m$. Then the map $\phi_{K, m}$ factors through a K-algebra isomorphism $\mathbf{H}_{K, m}^{Q_{t} ; q^{2}} \stackrel{\sim}{\longrightarrow} \mathscr{H}\left(K G_{n}, E_{t}\right)$.

4.4.3. Parametrization of the weak Harish-Chandra series of $\mathscr{U}_{K}$. Let $W\left(B_{m}\right)$ be the Weyl group of type $B_{m}$, and $t_{0}, t_{1}, \ldots, t_{m-1}$ be the generators corresponding to the following Dynkin diagram

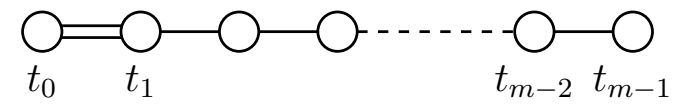

Let us first recall the construction of the irreducible characters of $W\left(B_{m}\right)$. We denote by $\sigma_{m}$ the linear character of $W\left(B_{m}\right)$ such that $\sigma_{m}\left(t_{0}\right)=-1$ and $\sigma_{m}\left(t_{i}\right)=1$ for all $i>0$. Given $\lambda \vdash m$ a partition of $m$, we write $\widetilde{\phi}_{\lambda}$ for the inflation to $W\left(B_{m}\right)$ of the irreducible character of $\mathfrak{S}_{m}$ corresponding to $\lambda$. Given $a, b$ such that $a+b=m$, one can consider the subgroups $W\left(B_{a}\right) \times \mathfrak{S}_{b} \subset W\left(B_{a}\right) \times W\left(B_{b}\right)$ of $W\left(B_{m}\right)$ where $W\left(B_{a}\right) \times$ $\mathfrak{S}_{b}$ is a parabolic subgroup generated by $\left\{t_{0}, t_{1}, \ldots, t_{a-1}\right\} \cup\left\{t_{a+1}, \ldots, t_{m-1}\right\}$, and $W\left(B_{a}\right) \times W\left(B_{b}\right)$ is obtained by adding the reflection $t_{a} \cdots t_{0} \cdots t_{a}$. The irreducible character of $W\left(B_{m}\right)$ associated to a bipartition $(\lambda, \mu)$ of $m$ is

$$
\mathscr{X}_{\lambda, \mu}=\operatorname{Ind}_{W\left(B_{|\lambda|}\right) \times W\left(B_{|\mu|}\right)}^{W\left(B_{m}\right)}\left(\widetilde{\phi}_{\lambda} \otimes \sigma_{|\mu|} \widetilde{\phi}_{\mu}\right) .
$$

By Tits deformation theorem, the specialization $q \mapsto 1$ yields a bijection

$$
\operatorname{Irr}\left(\mathbf{H}_{K, m}^{Q t ; q^{2}}\right) \stackrel{\sim}{\rightarrow} \operatorname{Irr}\left(W\left(B_{m}\right)\right)
$$


from which we obtain a canonical labelling of the irreducible representations of $\mathbf{H}_{K, m}^{Q_{t} ; q^{2}}$. We write $\operatorname{Irr}\left(\mathbf{H}_{K, m}^{Q_{t} ; q^{2}}\right)=\left\{S(\lambda, \mu)_{K}^{Q_{t} ; q^{2}}\right\}_{(\lambda, \mu) \in \mathscr{P}_{m}^{2}}$. Setting $T_{0}=(-1)^{t} q^{-1-t} X$, we have now the quadratic relation

$$
\left(T_{0}+1\right)\left(T_{0}-q^{2 t+1}\right)=0 .
$$

Using this generator instead of $X$, we obtain the usual presentation for a Hecke algebra of type $B_{m}$ with parameters $\left(q^{2 t+1}, q^{2}\right)$. The endomorphism of $K W\left(B_{m}\right)$ which is obtained from the renormalization $\mathbf{H}_{K, m}^{Q_{t} ; q^{2}} \stackrel{\sim}{\rightarrow} \mathbf{H}_{K, m}^{\left(q^{2 t+1}, 1\right) ; q^{2}}$ at $q=1$ is the identity on $\mathfrak{S}_{m}$ but sends $t_{0}$ to $(-1)^{t} t_{0}$. Therefore this renormalization sends $S(\lambda, \mu)$ to $S(\lambda, \mu)$ if $t$ is even, and to $S(\mu, \lambda)$ if $t$ is odd.

Corollary 4.9. Let $t, m \geqslant 0$ and $n=t(t+1) / 2+2 m$. Then the map $\phi_{K, m}$ and the functor $\mathfrak{F}_{F^{m}\left(E_{t}\right)}$ induce a bijection $\operatorname{WIrr}\left(K G_{n}, E_{t}\right) \stackrel{1: 1}{\longleftrightarrow} \operatorname{Irr}\left(\mathbf{H}_{K, m}^{Q_{t} ; q^{2}}\right)$ sending $E_{\lambda}$ to $S\left(\lambda^{[2]}\right)_{K}^{Q_{t}, q^{2}}$ for all partitions $\lambda \vdash n$ with 2 -core $\Delta_{t}=(t, t-1, \ldots, 1)$.

4.4.4. The $\mathfrak{g}_{\infty}$-representation on $\mathscr{U}_{K}$. The functors $E, F$ preserve the subcategory $\mathscr{U}_{K}$ by Proposition 3.6, hence $(E, F, X, T)$ yields a representation datum on $\mathscr{U}_{K}$. In order to extend it to a categorical representation on $\mathscr{U}_{K}$, one should consider the quiver $I\left(q^{2}\right)$ with vertices given by the various eigenvalues of $X$ and arrows $i \longrightarrow q^{2} i$. In this section we will view the integer $q$ as an element of $K^{\times}$in the obvious way.

Definition 4.10. Let $I_{\infty}$ denote the subset $(-q)^{\mathbb{Z}}$ of $K^{\times}$. We define $\mathfrak{g}_{\infty}$ to be the (derived) Kac-Moody algebra associated to the quiver $I_{\infty}\left(q^{2}\right)$.

To avoid cumbersome notation, we will write for short $I_{\infty}=I_{\infty}\left(q^{2}\right)$, and $(-)_{\infty}=$ $(-)_{I_{\infty}}$. We denote by $\left\{\Lambda_{i}\right\},\left\{\alpha_{i}\right\}$ and $\left\{\alpha_{i}^{\vee}\right\}$ the fundamental weights, simple roots and simple coroots of $\mathfrak{g}_{\infty}$. Here $\mathrm{X}_{\infty}$ coincides with $\mathrm{P}_{\infty}=\bigoplus \mathbb{Z} \Lambda_{i}$. Consequently, there is a Lie algebra isomorphism $\left(\mathfrak{s l}_{\mathbb{Z}}\right)^{\oplus 2} \stackrel{\sim}{\rightarrow} \mathfrak{g}_{\infty}$ such that $\left(\alpha_{d}^{\vee}, 0\right) \longmapsto \alpha_{-q^{2 d-1}}^{\vee}$ and $\left(0, \alpha_{d}^{\vee}\right) \longmapsto \alpha_{q^{2 d}}^{\vee}$

For any $t, m, n \in \mathbb{N}$, let $\left(K G_{n}, E_{t}\right)$-mod be the Serre subcategory of $\mathscr{U}_{K}$ generated by the modules $F^{m}\left(E_{t}\right)$ with $n=r+2 m$ and $r=t(t+1) / 2$. We define

$$
\mathscr{U}_{K, t}=\bigoplus_{n \geqslant 0}\left(K G_{n}, E_{t}\right)-\bmod
$$

Then $\mathscr{U}_{K}=\bigoplus_{t \geqslant 0} \mathscr{U}_{K, t}$. We obtain our first categorification result.

Theorem 4.11. Let $t \geqslant 0$ and $Q_{t}$ be as in (4.3).

(a) The Harish-Chandra induction and restriction functors yield a representation of $\mathfrak{g}_{\infty}$ on $\mathscr{U}_{K, t}$ which is isomorphic to $\mathscr{L}\left(\Lambda_{Q_{t}}\right)_{\infty}$.

(b) The map $\left|\mu, Q_{t}\right\rangle_{\infty} \longmapsto\left[E_{\varpi_{t}(\mu)}\right]$ induces an isomorphism of $\mathfrak{g}_{\infty}$-modules $\mathbf{F}\left(Q_{t}\right)_{\infty} \stackrel{\sim}{\rightarrow}$ $\left[\mathscr{U}_{K, t}\right]$. 


\subsection{The $\mathfrak{g}_{e}$-representation on $\mathscr{U}_{\mathrm{k}}$.}

By Proposition 3.6, the representation datum $(E, F, X, T)$ on $\mathrm{k} G$-mod induces a representation datum on $\mathscr{U}_{\mathrm{k}}$. Since the abelian category $\mathscr{U}_{\mathrm{k}}$ is not semisimple, to extend the representation datum to a categorical $\mathfrak{g}$-representation one needs to prove that weight spaces of $\mathscr{U}_{\mathrm{k}}$ are sums of blocks. This will be done combinatorially by studying a representation of a bigger Lie algebra $\mathfrak{g}_{e, \circ}$.

4.5.1. The Lie algebras $\mathfrak{g}_{e}$ and $\mathfrak{g}_{e, \mathrm{o}}$. The eigenvalues of $X$ on $E$ and $F$ are all powers of $-q$. If we denote again by $q$ the image of $q$ under the canonical map $\mathscr{O} \rightarrow \mathrm{k}$, then the eigenvalues of $X$ on $\mathrm{k} E$ and $\mathrm{k} F$ belong to the finite set $(-q)^{\mathbb{Z}} \subset \mathrm{k}^{\times}$. This set has exactly $e$ elements, where $e$ is the order of $-q$ in $\mathrm{k}^{\times}$.

Definition 4.12. We define $I_{e}$ to be the subset $(-q)^{\mathbb{Z}}$ of $\mathrm{k}^{\times}$. We denote by $I_{e}$ and $I_{e, \circ}$ the finite quivers $I_{e}\left(q^{2}\right)$ and $I_{e}(-q)$.

The quivers $I_{e}$ and $I_{e, \circ}$ have the same set of vertices, but the arrows in $I_{e}$ are the composition of two consecutive arrows in $I_{e, \circ}$. The quiver $I_{e, \circ}$ is cyclic, whereas the quiver $I_{e}$ is cyclic if $e$ is odd, and is a union of two cyclic quivers if $e$ is even. Therefore the corresponding Kac-Moody algebras are isomorphic to $\widehat{\mathfrak{s l}}_{e}$ or $\left(\widehat{\mathfrak{s l}}_{e / 2}\right)^{\oplus 2}$.

To avoid cumbersome notation, we will write $(\bullet)_{e, \circ}=(\bullet)_{I_{e, \circ}}$ and $(\bullet)_{e}=(\bullet)_{I_{e}}$. We must introduce Lie algebras $\mathfrak{g}_{e, \circ}$ and $\mathfrak{g}_{e}$ such that $\mathfrak{g}_{e, \circ}^{\prime}=\left[\mathfrak{g}_{e, \mathrm{o}}, \mathfrak{g}_{e, \mathrm{o}}\right]$ and $\mathfrak{g}_{e}^{\prime}=\left[\mathfrak{g}_{e}, \mathfrak{g}_{e}\right]$. The Chevalley generators of $\mathfrak{g}_{e, \circ}^{\prime}$ and $\mathfrak{g}_{e}^{\prime}$ are $e_{i, \circ}, f_{i, \circ}$ and $e_{i}, f_{i}$ respectively, for $i \in$ $(-q)^{\mathbb{Z}}$. There is a Lie algebra homomorphism $\kappa: \mathfrak{g}_{e}^{\prime} \longrightarrow \mathfrak{g}_{e, \circ}^{\prime}$ defined by

$$
\kappa\left(e_{i}\right)=\left[e_{-q i, o}, e_{i, o}\right] \text { and } \kappa\left(f_{i}\right)=\left[f_{-q i, \circ}, f_{i, o}\right] .
$$

It restricts to a map between the coroot lattices sending $\alpha_{i}^{\vee}$ to $\alpha_{i, \circ}^{\vee}+\alpha_{-q i, \circ}^{\vee}$

We denote by $\mathfrak{g}_{e, \circ}$ the Kac-Moody algebra associated with the lattices $\mathrm{X}_{e, 0}=$ $\mathrm{P}_{e, \circ} \oplus \mathbb{Z} \delta_{\circ}$ and $\mathrm{X}_{e, \circ}^{\vee}=\mathrm{Q}_{e, \circ}^{\vee} \oplus \mathbb{Z} \partial_{\circ}$, where $\delta_{\circ}=\sum \alpha_{i, \circ}, \partial_{\circ}=\Lambda_{1, \circ}^{\vee}$ and the pairing $\mathrm{X}_{e, 0}^{\vee} \times \mathrm{X}_{e, 0} \longrightarrow \mathbb{Z}$ is given by

$$
\left\langle\alpha_{j, \circ}^{\vee}, \Lambda_{i, \circ}\right\rangle_{e, \circ}=\delta_{i j}, \quad\left\langle\partial_{\circ}, \Lambda_{i, \circ}\right\rangle_{e, \circ}=\left\langle\alpha_{j, \circ}^{\vee}, \delta_{\circ}\right\rangle_{e, \circ}=0, \quad\left\langle\partial_{\circ}, \delta_{\circ}\right\rangle_{e, \circ}=1 .
$$

Then $\mathfrak{g}_{e, \circ}, \mathfrak{g}_{e, \circ}^{\prime}$ are isomorphic to $\widehat{\mathfrak{s l}}_{e}, \widetilde{\mathfrak{s l}}_{e}$ respectively.

Let $\widetilde{\mathfrak{g}}_{e}$ be the usual Kac-Moody algebra associated with $I_{e}$. Its derived Lie subalgebra is equal to $\mathfrak{g}_{e}^{\prime}$. Let $\widetilde{\mathrm{X}}_{e}$ and $\widetilde{\mathrm{X}}_{e}^{\vee}$ be the lattices corresponding to $\widetilde{\mathfrak{g}}_{e}$. The map $\kappa: \mathfrak{g}_{e}^{\prime} \longrightarrow \mathfrak{g}_{e, \circ}^{\prime}$ may not extend to a morphism of Lie algebras $\widetilde{\mathfrak{g}}_{e} \longrightarrow \mathfrak{g}_{e, \circ}$. For this reason we will define $\mathfrak{g}_{e}$ to be the Lie subalgebra $\mathfrak{g}_{e}^{\prime} \oplus \mathbb{C} \partial$ of $\widetilde{\mathfrak{g}}_{e}$, where $\partial$ is the element given by $\partial=\Lambda_{1}^{\vee}+\Lambda_{-q^{-1}}^{\vee}$. We can view $\mathfrak{g}_{e}$ as the Kac-Moody algebra associated with the lattice $\mathrm{X}_{e}^{\vee}=\mathrm{Q}_{e}^{\vee} \oplus \mathbb{Z} \partial$ above and a lattice $\mathrm{X}_{e}=\mathrm{P}_{e} \oplus \mathbb{Z} \delta$ that we define below case-by-case. 
If $e$ is odd, then $I_{e}$ is a cyclic quiver and $\mathfrak{g}_{e}=\widetilde{\mathfrak{g}}_{e}$ is isomorphic to $\widehat{\mathfrak{s l}}_{e}$. Let $\alpha_{1}$ be the affine root, then we have $\widetilde{\mathrm{X}}_{e}=\mathrm{P}_{e} \oplus \mathbb{Z} \tilde{\delta}$ and $\widetilde{\mathrm{X}}_{e}^{\vee}=\mathrm{Q}_{e}^{\vee} \oplus \mathbb{Z} \widetilde{\partial}$ with $\tilde{\delta}=\sum \alpha_{i}$ and $\widetilde{\partial}=\Lambda_{1}^{\vee}$. Then, we set $\mathrm{X}_{e}=\mathrm{P}_{e} \oplus \mathbb{Z} \delta$ and $\delta=\tilde{\delta} / 2$.

If $e$ is even, then $I_{e}$ is the disjoint union of two cyclic quivers and $\tilde{\mathfrak{g}}_{e}$ is isomorphic to $\left(\widehat{\mathfrak{s l}}_{e / 2}\right)^{\oplus 2}$. Let $\alpha_{1}$ and $\alpha_{-q^{-1}}$ be the affine roots, then we have $\widetilde{\mathrm{X}}_{e}=\mathrm{P}_{e} \oplus \mathbb{Z} \delta_{1} \oplus \mathbb{Z} \delta_{2}$ and $\widetilde{\mathrm{X}}_{e}^{\vee}=\mathrm{Q}_{e}^{\vee} \oplus \mathbb{Z} \partial_{1} \oplus \mathbb{Z} \partial_{2}$ with $\delta_{1}=\sum_{j \text { odd }} \alpha_{-q^{j}}, \delta_{2}=\sum_{j \text { even }} \alpha_{q^{j}}, \partial_{1}=\Lambda_{-q^{-1}}^{\vee}$ and $\partial_{2}=\Lambda_{1}^{\vee}$. Then, we set $\mathrm{X}_{e}=\widetilde{\mathrm{X}}_{e} /\left(\delta_{1}-\delta_{2}\right)$ and $\delta=\left(\delta_{1}+\delta_{2}\right) / 2$.

In both cases the perfect pairing $\mathrm{X}_{e}^{\vee} \times \mathrm{X}_{e} \longrightarrow \mathbb{Z}$ is induced in the obvious way by the pairing $\widetilde{\mathrm{X}}_{e}^{\vee} \times \widetilde{\mathrm{X}}_{e} \longrightarrow \mathbb{Z}$.

Lemma 4.13. There is a well-defined morphism of Lie algebras $\mathfrak{g}_{e} \longrightarrow \mathfrak{g}_{e, \circ}$ which extends $\kappa$ and whose restriction to $\mathrm{X}_{e}^{\vee}$ is given by $\kappa\left(\alpha_{i}^{\vee}\right)=\alpha_{i, \circ}^{\vee}+\alpha_{-q i, \circ}^{\vee}$ and $\kappa(\partial)=\partial_{\circ}$. The restriction $\kappa: \mathrm{X}_{e}^{\vee} \longrightarrow \mathrm{X}_{e, \circ}^{\vee}$ has an adjoint $\kappa^{*}: \mathrm{X}_{e, \circ} \longrightarrow \mathrm{X}_{e}$ such that $\kappa^{*}\left(\Lambda_{i, \circ}\right) \equiv$ $\Lambda_{i}+\Lambda_{-q^{-1} i} \bmod \delta$ and $\kappa^{*}\left(\delta_{\circ}\right)=\delta$.

4.5.2. The $\mathfrak{g}_{e}^{\prime}$-action on $\left[\mathscr{U}_{\mathrm{k}}\right]$. The quotient map $\mathscr{O} \rightarrow \mathrm{k}$ induces a morphism of quivers sp $: I_{\infty} \longrightarrow I_{e}$ and a surjective morphism of abelian groups $\mathrm{sp}: \mathrm{P}_{\infty} \rightarrow$ $\mathrm{P}_{e}$ sending $\Lambda_{i}$ to $\Lambda_{\mathrm{sp}(i)}$. In addition any integrable representation $V$ of $\mathfrak{g}_{\infty}$ can be "restricted" to an integrable representation of the derived algebra $\mathfrak{g}_{e}^{\prime}$, where $e_{i} \in \mathfrak{g}_{e}^{\prime}$ (resp. $f_{i} \in \mathfrak{g}_{e}^{\prime}$ ) act as $\sum_{\mathrm{sp}(j)=i} e_{j}$ (resp. $\sum_{\mathrm{sp}(j)=i} f_{j}$ ). From the definition of the action of $\mathfrak{g}_{\infty}$ and $\mathfrak{g}_{e}$ on Fock spaces, see (2.2), we deduce that the map $\left|\mu, Q_{t}\right\rangle_{\infty} \mapsto\left|\mu, Q_{t}\right\rangle_{e}$ induces the following isomorphism of $\mathfrak{g}_{e}^{\prime}$-modules

$$
\text { sp : } \operatorname{Res}_{\mathfrak{g}_{e}^{\prime}}^{\mathfrak{g}_{\infty}} \mathbf{F}\left(Q_{t}\right)_{\infty} \stackrel{\sim}{\longrightarrow} \mathbf{F}\left(Q_{t}\right)_{e} .
$$

Under the decomposition map, this isomorphism endows $\left[\mathscr{U}_{\mathrm{k}}\right]$ with a structure of $\mathfrak{g}_{e}^{\prime}$-module which is compatible with the one coming from the representation datum.

Proposition 4.14. For each $i \in I_{e}$, let $\mathrm{k} E_{i}$ (resp. $\mathrm{k} F_{i}$ ) be the generalized $i$-eigenspace of $X$ on $\mathrm{k} E$ (resp. $\mathrm{k} F)$.

(a) $\left[\mathrm{k} E_{i}\right],\left[\mathrm{k} F_{i}\right]$ endow $\left[\mathscr{U}_{\mathrm{k}}\right]$ with a structure of $\mathfrak{g}_{e}^{\prime}$-module.

(b) $d_{\mathscr{U}}:\left[\mathscr{U}_{K}\right] \longrightarrow\left[\mathscr{U}_{k}\right]$ induces a $\mathfrak{g}_{e}^{\prime}$-module isomorphism $\operatorname{Res}_{\mathfrak{g}_{e}^{\prime}}^{\mathfrak{g}_{\infty}}\left[\mathscr{U}_{K}\right] \stackrel{\sim}{\rightarrow}\left[\mathscr{U}_{k}\right]$.

(c) $\left|\mu, Q_{t}\right\rangle_{e} \longmapsto\left[V_{\varpi_{t}(\mu)}\right]$ induces a $\mathfrak{g}_{e}^{\prime}$-module isomorphism $\bigoplus_{t \in \mathbb{N}} \mathbf{F}\left(Q_{t}\right)_{e} \stackrel{\sim}{\longrightarrow}\left[\mathscr{U}_{\mathrm{k}}\right]$.

4.5.3. The $\mathfrak{g}_{e}$-action on $\left[\mathscr{U}_{\mathrm{k}}\right]$. We now define an action of $\mathfrak{g}_{e}$ on $\left[\mathscr{U}_{\mathrm{k}}\right]$ by extending the action from $\mathfrak{g}_{e}^{\prime}$ to $\mathfrak{g}_{e}$ on $\bigoplus_{t \in \mathbb{N}} \mathbf{F}\left(Q_{t}\right)_{e}$. This amounts to extending the grading from $\mathrm{P}_{e}$ to $\mathrm{X}_{e}=\mathrm{P}_{e} \oplus \mathbb{Z} \delta$. 
Any integrable $\mathfrak{g}_{e, \circ}$-representation (resp. $\mathfrak{g}_{e, \mathrm{o}}^{\prime}$-representation) can be "restricted" to an integrable $\mathfrak{g}_{e}$-representation (resp. $\mathfrak{g}_{e}^{\prime}$-representation) through $\kappa$. We denote by $\operatorname{Res}_{\mathfrak{g}_{e}}^{\mathfrak{g}_{e, 0}}$ and $\operatorname{Res}_{\mathfrak{g}_{e}^{\prime}}^{\mathfrak{g}_{e}^{\prime}, \mathrm{e}}$ the corresponding operations.

Let $a: \mathscr{P} \rightarrow \mathbb{N}$ be Lusztig's $a$-function, see [54, 4.4.2]. Recall that $a(\lambda)=$ $\sum_{i}(i-1) \lambda_{i}$ if $\lambda=\left(\lambda_{1}, \lambda_{2}, \ldots\right)$.

Lemma 4.15. The assignment $\left|\mu, Q_{t}\right\rangle_{e} \longmapsto(-1)^{a\left(\varpi_{t}(\mu)\right)}\left|\varpi_{t}(\mu), 1\right\rangle_{e, \circ}$ induces an isomorphism of $\mathfrak{g}_{e}^{\prime}$-modules $\bigoplus_{t \in \mathbb{N}} \mathbf{F}\left(Q_{t}\right)_{e} \stackrel{\sim}{\longrightarrow} \operatorname{Res}_{\mathfrak{g}_{e}^{\prime}}^{\mathfrak{g}_{e}^{\prime}} \mathbf{F}(1)_{e, \circ}$.

Now, consider the Fock space $\mathbf{F}(1)_{e, o}$ as a charged Fock space for the charge $s=0$. This endows $\mathbf{F}(1)_{e, \circ}$ with an integrable representation of $\mathfrak{g}_{e, \circ}$ as in $\$ 2.3 .1$. Consequently, we can endow $\bigoplus_{t \in \mathbb{N}} \mathbf{F}\left(Q_{t}\right)_{e}$ and $\left[\mathscr{U}_{\mathrm{k}}\right]$ with integrable representations of $\mathfrak{g}_{e}$ such that there are $\mathfrak{g}_{e}$-module isomorphisms

$$
\begin{aligned}
& \bigoplus_{t \in \mathbb{N}} \mathbf{F}\left(Q_{t}\right)_{e} \stackrel{\sim}{\longrightarrow}\left[\mathscr{U}_{\mathrm{k}}\right] \stackrel{\sim}{\longrightarrow} \operatorname{Res}_{\mathfrak{g}_{e}}^{\mathfrak{g}_{e, o}} \mathbf{F}(1)_{e, \circ} \\
& \left|\mu, Q_{t}\right\rangle_{e} \mapsto\left[V_{\varpi_{t}(\mu)}\right] \mapsto(-1)^{a\left(\varpi_{t}(\mu)\right)}\left|\varpi_{t}(\mu), 1\right\rangle_{e, \circ} .
\end{aligned}
$$

4.5.4. The $\mathfrak{g}_{e}$-action on $\mathscr{U}_{\mathrm{k}}$. The following lemma holds.

Lemma 4.16. Let $\lambda$ and $\mu$ be partitions of $n$. If $V_{\mu}, V_{\lambda}$ belong to the same block of $\mathscr{U}_{\mathrm{k}}$ then $\left[V_{\lambda}\right],\left[V_{\mu}\right]$ have the same weight for the action of $\mathfrak{g}_{e}$.

We deduce that the classes of the simple modules $\left[D_{\lambda}\right]$ are also weight vectors. Given $\omega \in \mathrm{X}_{e}$, we define $\mathscr{U}_{\mathrm{k}, \omega}$ to be the Serre subcategory of $\mathscr{U}_{\mathrm{k}}$ generated by the simple modules $D_{\lambda}$ such that the element $\left[D_{\lambda}\right]$ in $\left[\mathscr{U}_{\mathrm{k}}\right]$ has weight $\omega$. We have $\mathscr{U}_{\mathrm{k}}=\bigoplus_{\omega \in \mathrm{X}_{e}} \mathscr{U}_{\mathrm{k}, \omega}$. We can now formulate the following.

Theorem 4.17. The representation datum associated with Harish-Chandra induction and restriction and the decomposition $\mathscr{U}_{\mathrm{k}}=\bigoplus_{\omega \in \mathrm{X}_{e}} \mathscr{U}_{\mathrm{k}, \omega}$ yield a categorical representation of $\mathfrak{g}_{e}$ on $\mathscr{U}_{\mathrm{k}}$. Furthermore, the map $\left|\mu, Q_{t}\right\rangle_{e} \longmapsto\left[V_{\varpi_{t}(\mu)}\right]$ induces a $\mathfrak{g}_{e}^{\prime}$-module isomorphism $\bigoplus_{t \in \mathbb{N}} \mathbf{F}\left(Q_{t}\right)_{e} \stackrel{\sim}{\rightarrow}\left[\mathscr{U}_{\mathrm{k}}\right]$.

\subsection{Derived equivalences of blocks of $\mathscr{U}_{\mathrm{k}}$.}

Recall that $d, e$ and $f$ denote respectively the order of $q^{2},-q$ and $q$ modulo $\ell$. 
4.6.1. Characterization of the blocks of $\mathscr{U}_{\mathrm{k}}$. Here we investigate which block can occur in a given weight space.

Assume $e$ is even. Given $t \in \mathbb{N}$ and $\omega \in \mathrm{X}_{e}$, we define $\mathscr{U}_{\mathrm{k}, t}$ (resp. $\mathscr{U}_{\mathrm{k}, t, \omega}$ ) to be the Serre subcategory of $\mathscr{U}_{\mathrm{k}}$ generated by simple modules $D_{\lambda}$ where $\lambda$ has 2-core $\Delta_{t}$ (resp. with in addition $\omega_{\lambda}=\omega$ ). With $e$ being even, any pair of partitions with the same $e$-core have the same 2 -core, therefore $\mathscr{U}_{\mathrm{k}, t}$ is a direct summand of $\mathscr{U}_{\mathrm{k}}$.

Proposition 4.18. Let $\omega \in \mathrm{X}_{e}$.

(a) If e is odd, then the category $\mathscr{U}_{\mathrm{k}, \omega}$ is an indecomposable summand of $\mathscr{U}_{\mathrm{k}}$.

(b) If e is even, then the category $\mathscr{U}_{\mathrm{k}, t, \omega}$ is an indecomposable summand of $\mathscr{U}_{\mathrm{k}, t}$ and $\mathscr{U}_{\mathrm{k}}$ for all $t \in \mathbb{N}$.

4.6.2. Derived equivalences of blocks of $\mathscr{U}_{\mathrm{k}}$. It is not difficult to compute the orbits of the affine Weyl group on the weight spaces of $\left[\mathscr{U}_{\mathrm{k}}\right]$. Hence, we can apply Proposition 4.18 and the work of Chuang and Rouquier [10] to produce derived equivalences between blocks of $\mathscr{U}_{\mathrm{k}}$ in the same orbit. Using the results of Livesey [51] on the structure of good blocks for linear primes, we deduce that Broué's abelian defect group conjecture holds for unipotent blocks when $e$ is even.

Theorem 4.19. Assume $e$ is even. Let $B$ be a unipotent block of $G_{n}$ over $\mathrm{k}$ or $\mathscr{O}$, and $D$ be a defect group of $B$. If the group $D$ is abelian, then the block $B$ is derived equivalent to its Brauer correspondent in $N_{G_{n}}(D)$.

\subsection{The crystals of $\mathscr{U}_{K}$ and $\mathscr{U}_{\mathrm{k}}$.}

Now, we want to compare the crystals of the categorical representations $\mathscr{U}_{K}$ and $\mathscr{U}_{\mathrm{k}}$ (which are related to Harish-Chandra induction and restriction) with the crystals of the Fock spaces related to $\left[\mathscr{U}_{K}\right]$ and $\left[\mathscr{U}_{\mathrm{k}}\right]$. This solves the main conjecture of GerberHiss-Jacon [36] and gives a combinatorial way to compute the (weak) Harish-Chandra branching graph and the Hecke algebras associated to the weakly cuspidal unipotent modules.

4.7.1. Crystals and Harish-Chandra series. Recall that to any categorical representation one can associate a perfect basis, and hence an abstract crystal. In the previous sections we constructed a categorical action on the categories of unipotent representations over $K$ (denoted by $\mathscr{U}_{K}$ ) and over $\mathrm{k}$ (denoted by $\mathscr{U}_{k}$ ). From these two categorical representations we get the following abstract crystals

(a) $B\left(\mathscr{U}_{K}\right)=\left(\operatorname{Irr}\left(\mathscr{U}_{K}\right), \widetilde{E}_{i}, \widetilde{F}_{i}\right)$ where $\operatorname{Irr}\left(\mathscr{U}_{K}\right)=\left\{\left[E_{\lambda}\right] \mid \lambda \in \mathscr{P}\right\}$,

(b) $B\left(\mathscr{U}_{\mathrm{k}}\right)=\left(\operatorname{Irr}\left(\mathscr{U}_{\mathrm{k}}\right), \widetilde{E}_{i}, \widetilde{F}_{i}\right)$ where $\operatorname{Irr}\left(\mathscr{U}_{\mathrm{k}}\right)=\left\{\left[D_{\lambda}\right] \mid \lambda \in \mathscr{P}\right\}$. 
The (uncolored) crystal graph associated with $B\left(\mathscr{U}_{R}\right)$ coincides with the weak HarishChandra branching graph, and its connected components with the weak HarishChandra series.

Proposition 4.20. Let $R=K$ or $\mathrm{k}$, and $I=(-q)^{\mathbb{Z}} \subset R^{\times}$. Let $D, M, N \in \operatorname{Irr}\left(\mathscr{U}_{R}\right)$.

(a) $D$ is weakly cuspidal if and only if $\widetilde{E}_{i}(D)=0$ for all $i \in I$.

(b) $N$ appears in $\operatorname{hd}(F(M))$ if and only if there exists $i \in I$ such that $N \simeq \widetilde{F}_{i} M$.

(c) If $D$ is weakly cuspidal, then

$$
\operatorname{WIrr}(R G, D)=\left\{\widetilde{F}_{i_{1}} \cdots \widetilde{F}_{i_{m}}(D) \mid m \in \mathbb{N}, i_{1}, \ldots, i_{m} \in I\right\}
$$

Note that by [53], the ordinary Harish-Chandra series and weak Harish-Chandra series on unipotent modules coincide when $R=K$.

4.7.2. Comparison of the crystals. In this section we will assume that $e$ is odd. To any charged Fock space one can associate an abstract crystal, see \$2.3.2. We now show how to choose the charge for each Fock space $\mathbf{F}\left(Q_{t}\right)_{e}$ so that the crystal will coincide with the Harish-Chandra branching graph.

Define $s_{t}=\left(s_{1}, s_{2}\right)=-\frac{1}{2}(e+1,0)+\sigma_{t}$ where $\sigma_{t}$ is as in (2.1). We have $Q_{p}=q^{2 s_{p}}$ for each $p=1,2$. We denote by $B\left(s_{t}\right)_{e}$ the corresponding abstract crystal of $\mathbf{F}\left(Q_{t}\right)_{e}$, with the canonical labeling $B\left(s_{t}\right)_{e}=\left\{b\left(\mu, s_{t}\right) \mid \mu \in \mathscr{P}^{2}, t \in \mathbb{N}\right\}$. Finally, we set $B_{e}=\bigsqcup_{t \in \mathbb{N}} B\left(s_{t}\right)_{e}$.

Theorem 4.21. The map $b\left(\mu, s_{t}\right) \mapsto\left[D_{\varpi_{t}(\mu)}\right]$ is a crystal isomorphism $B_{e} \stackrel{\sim}{\rightarrow} B\left(\mathscr{U}_{\mathrm{k}}\right)$.

We deduce the following corollaries.

Corollary 4.22. The modules $D_{\lambda}$ and $D_{\nu}$ lie in the same weak Harish-Chandra series if and only if the corresponding vertices of the abstract crystal $B_{e}$ belong to the same connected component. In particular, if this holds then $\lambda$ and $\nu$ have the same 2-core.

Corollary 4.23. Let $\lambda$ be a partition of $r>0$ such that $D_{\lambda}$ is weakly cuspidal.

(a) The e-core of $\lambda$ is a 2-core $\Delta_{t}=(t, t-1, \ldots, 1)$,

(b) the weight of the class $\left[D_{\lambda}\right]$ with respect to the $\mathfrak{g}_{e}^{\prime}$-action on $\left[\mathscr{U}_{\mathrm{k}}\right]$ is $\Lambda_{Q_{t}}$,

(c) for each $m \geqslant 0$ and $n=r+2 m$, the $\operatorname{map} \phi_{\mathrm{k}, m}: \mathbf{H}_{\mathrm{k}, m}^{q^{2}} \longrightarrow \operatorname{End}_{\mathrm{k} G_{n}}\left(F^{m}\right)^{\mathrm{op}}$ factors through an algebra isomorphism $\mathbf{H}_{\mathrm{k}, m}^{Q_{t} ; q^{2}} \stackrel{\sim}{\longrightarrow} \mathscr{H}\left(\mathrm{k} G_{n}, D_{\lambda}\right)$. 


\section{The representation of the Heisenberg algebra on $\mathscr{U}_{\mathrm{k}}$}

Every cuspidal $\mathrm{k} G_{n}$-module is weakly cuspidal. Therefore, every Harish-Chandra series in $\operatorname{Irr}\left(\mathscr{U}_{\mathrm{k}}\right)$ is partitioned into weak Harish-Chandra series. Proposition 4.20 and Theorem 4.21 yield a complete (combinatorial) description of the partition of $\operatorname{Irr}\left(\mathscr{U}_{\mathrm{k}}\right)$ into weak Harish-Chandra series using the decomposition of $\left[\mathscr{U}_{\mathrm{k}}\right]$ for the action of $\mathfrak{g}_{e}$. In this section we generalize this result to usual Harish-Chandra series by adding the action of some Heisenberg algebra on $\left[\mathscr{U}_{\mathrm{k}}\right]$. Throughout this section we will assume that $e$, the order of $-q$ modulo $\ell$ is odd, and hence equal to $d$, the order of $q^{2}$ modulo $\ell$.

\subsection{The Heisenberg action on $\left[\mathscr{U}_{\mathrm{k}}\right]$.}

5.1.1. The Heisenberg algebra. Set $\Lambda=\bigoplus_{m \in \mathbb{N}} \mathbb{C} \operatorname{Irr}\left(K \mathfrak{S}_{m}\right)$ where $\mathfrak{S}_{0}$ is the trivial group. We equip the vector space $\Lambda$ with the bilinear form $\langle\bullet, \bullet\rangle_{\Lambda}=\bigoplus_{m \in \mathbb{N}}\langle\bullet, \bullet\rangle_{\mathfrak{S}_{m}}$ and the vector space $\Lambda \otimes \Lambda$ with the tensor square of $\langle\bullet, \bullet\rangle_{\Lambda}$. The induction and restriction yield a pair of linear maps $\operatorname{Ind}_{n, m}^{n+m}$ and $\operatorname{Res}_{n, m}^{n+m}$ between the $\mathbb{C}$-vector spaces $\Lambda$ and $\Lambda \otimes \Lambda$ which are adjoint with respect to these bilinear forms.

Fix a positive integer $a$. A level $a$ representation of the Heisenberg algebra, or an $\mathfrak{H}_{a}$-module, is a $\mathbb{C}$-vector space $V$ with a family of endomorphisms $b_{n}, b_{n}^{*}$ labelled by integers $n \in a \mathbb{Z}_{>0}$ satisfying the relations $\left[b_{n}, b_{m}\right]=\left[b_{n}^{*}, b_{m}^{*}\right]=0$ and $\left[b_{n}, b_{m}^{*}\right]=$ $-n \delta_{n, m}$. For each partition $\lambda$ we write $a \lambda=\left(a \lambda_{1}, a \lambda_{2}, \ldots\right)$ and $b_{a \lambda}=\prod_{i} b_{a \lambda_{i}}$, $b_{a \lambda}^{*}=\prod_{i} b_{a \lambda_{i}}^{*}$.

If $\lambda$ is a partition of $m$ and $w \in \mathfrak{S}_{m}$ is of cycle-type $\rho(w)=\lambda$, we denote by $c_{\lambda}=c_{w}$ the conjugacy class of $w$. The characteristic function of $c_{\lambda}$ is a class function on $\mathfrak{S}_{m}$, and as such it can be viewed as an element in $\Lambda$. We abbreviate $c_{m}=c_{(m)}$ for the conjugacy class of the $m$-cycles. Let $z_{\lambda}$ be the number of elements of the centralizer of $w$ in $\mathfrak{S}_{m}$. We have $\left\langle c_{\lambda}, c_{\mu}\right\rangle=z_{\lambda}^{-1} \delta_{\lambda, \mu}$.

There is a unique representation of $\mathfrak{H}_{1}$ on $\Lambda$ such that for every $\phi \in \mathbb{C} \operatorname{Irr}\left(\mathfrak{S}_{r}\right)$ and $\psi \in \mathbb{C} \operatorname{Irr}\left(\mathfrak{S}_{n}\right)$ with $n=r+m$ we have

$$
b_{\lambda}(\phi)=\operatorname{Ind}_{r, m}^{n}\left(\phi \otimes z_{\lambda} c_{\lambda}\right), \quad b_{\lambda}^{*}(\psi)=\left\langle\operatorname{Res}_{r, m}^{n}(\psi), z_{\lambda} c_{\lambda}\right\rangle_{\Lambda} .
$$

5.1.2. The Heisenberg action on $\mathbf{F}(Q)$. Recall that $e$ is the order of $-q$ modulo $\ell$. We defined in $\$ 4.5 .1$ a Kac-Moody algebra $\mathfrak{g}_{e}$ corresponding to a quiver $I_{e}$. From now one we will assume that $e$ is odd and we will abbreviate $\mathfrak{g}=\mathfrak{g}_{e}$. Then $\mathfrak{g}$ is isomorphic to $\widehat{\mathfrak{s l}}_{e}$. We can now define the representation of $\mathfrak{H}_{l e}$ on $\mathbf{F}(Q)$, where $Q=\left(Q_{1}, \ldots, Q_{l}\right) \in I_{e}^{l}$. The Fock space $\mathbf{F}(Q)$ admits a representation of $\mathfrak{H}_{l}$ which is identified with the $l$-th tensor power of the representation of $\mathfrak{H}_{1}$ on $\Lambda$ in (5.1) under the $\mathbb{C}$-linear isomorphism $\mathbf{F}(Q) \stackrel{\sim}{\rightarrow} \boldsymbol{\Lambda}^{\otimes l}$ such that $|\mu, Q\rangle \mapsto \phi_{\mu^{1}} \otimes \cdots \otimes \phi_{\mu^{l}}$. Then, by 
(2.2), the representation of $\mathfrak{g}^{\prime}$ on $\mathbf{F}(Q)$ commutes with the action of the subalgebra $\mathfrak{H}_{l e}$ of $\mathfrak{H}_{l}$. We will apply this construction to $l=1$ or 2 .

5.1.3. The Heisenberg action on $\left[\mathscr{U}_{\mathrm{k}}\right]$. The previous section yields a representation of $\mathfrak{H}_{2 e}$ in $\mathbf{F}\left(Q_{t}\right)_{e}$ for each $t \in \mathbb{N}$ and a representation of $\mathfrak{H}_{e}$ in $\mathbf{F}(1)_{e, o}$. We want to compare them.

To do that, consider the $\mathbb{C}$-linear isomorphism $\bigoplus_{t \in \mathbb{N}} \mathbf{F}\left(Q_{t}\right)_{e} \stackrel{\sim}{\rightarrow} \mathbf{F}(1)_{e, o}$ studied in Lemma 4.15, which is given by $\left|\mu, Q_{t}\right\rangle_{e} \mapsto(-1)^{a(\lambda)}|\lambda, 1\rangle_{e, \circ}$ with $\lambda=\varpi_{t}(\mu)$.

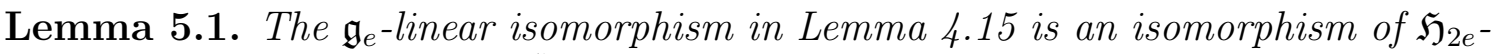
modules $\bigoplus_{t \in \mathbb{N}} \mathbf{F}\left(Q_{t}\right)_{e} \stackrel{\sim}{\longrightarrow} \operatorname{Res}_{\mathfrak{H}_{2 e}}^{\mathfrak{H}_{e}} \mathbf{F}(1)_{e, o}$.

Proof. Fix an integer $t \geqslant 0$. We must check that the linear map

$$
\mathbf{F}\left(Q_{t}\right)_{e} \simeq \boldsymbol{\Lambda} \otimes \boldsymbol{\Lambda} \rightarrow \boldsymbol{\Lambda}
$$

such that

$$
\left|\mu, Q_{t}\right\rangle_{e} \mapsto \phi_{\mu^{1}} \otimes \phi_{\mu^{2}} \mapsto(-1)^{a(\lambda)} \phi_{\lambda}, \quad \forall \mu \in \mathscr{P}^{2}
$$

intertwines the operator $b_{n} \otimes 1+1 \otimes b_{n}$ on $\Lambda \otimes \Lambda$ with the operator $b_{2 n}$ on $\Lambda$ if $n \in e \mathbb{Z}_{>0}$. Here, we have set $\lambda=\varpi_{t}(\mu)$.

To do this, note that we have

$$
b_{2 n}\left(\phi_{\lambda}\right)=\sum_{\nu}(-1)^{N(\lambda, x, 2 n)} \phi_{\nu}
$$

where the sum runs over all partitions $\nu$ obtained by adding a $2 n$-hook $(x, x+2 n)$ to the charged partition $(\lambda, 0)$ and $N(\lambda, x, 2 n)$ is the number of elements in $\beta_{0}(\lambda) \cap$ $(x, x+2 n)$. Recall that $\sigma_{t}=\left(\sigma_{1}, \sigma_{2}\right)$. Since

$$
\beta_{0}(\lambda)=\left(-1+2 \beta_{\sigma_{1}}\left(\mu^{1}\right)\right) \sqcup\left(2 \beta_{\sigma_{2}}\left(\mu^{2}\right)\right),
$$

we have

$$
N(\lambda, x, 2 n)= \begin{cases}N\left(\mu^{1}, y-\sigma_{1}, n\right)+\left|\left(2 \beta_{\sigma_{2}}\left(\mu^{2}\right)\right) \cap(x, x+2 n)\right| & \text { if } x=-1+2 y, \\ \left|\left(-1+2 \beta_{\sigma_{1}}\left(\mu^{1}\right)\right) \cap(x, x+2 n)\right|+N\left(\mu^{2}, y-\sigma_{2}, n\right) & \text { if } x=2 y .\end{cases}
$$

Furthermore, adding a $2 n$-hook $(x, x+2 n)$ to $\lambda$ is equivalent to adding an $n$-hook to $\mu^{1}$ if $x$ is odd or to $\mu^{2}$ if $x$ is even. So we are reduced to check that if $\lambda, \nu, x$ are as in the sum (5.2), then $a(\nu)-a(\lambda)$ has the same parity as the integer $b(\lambda, x)$ given by

- $\left|\left(2 \beta_{\sigma_{2}}\left(\mu^{2}\right)\right) \cap(x, x+2 n)\right|$ if $x$ is odd,

$$
\text { - }\left|\left(-1+2 \beta_{\sigma_{1}}\left(\mu^{1}\right)\right) \cap(x, x+2 n)\right| \text { if } x \text { is even. }
$$

Now a partition $\nu$ obtained by adding a $2 n$-hook to $\lambda$ can also be obtained by adding $n$ successive 2 -hooks to $\lambda$. Therefore it is enough to check that the parity of $a(\nu)-a(\lambda)$ and $b(\lambda, x)$ are the same when $n=1$. Since $a(\lambda)=\sum_{i}(i-1) \lambda_{i}$, the integer $a(\nu)-a(\lambda)$ is even if $\nu \backslash \lambda$ is a vertical 2-hook (increasing $\lambda_{i}$ by 2 for some 
$i$ ) or odd if $\nu \backslash \lambda$ is a horizontal 2-hook (increasing $\lambda_{i}$ and $\lambda_{i+1}=\lambda_{i}$ by 1 for some $i)$. On the other hand, we have $b(\lambda, x)=0(\operatorname{resp} . b(\lambda, x)=1)$ for a vertical 2 -hook (resp. for a horizontal 2-hook).

We equip $\left[\mathscr{U}_{\mathrm{k}}\right]$ with the unique representation of $\mathfrak{H}_{2 e}$ such that the isomorphism $\bigoplus_{t \in \mathbb{N}} \mathbf{F}\left(Q_{t}\right)_{e} \stackrel{\sim}{\rightarrow}\left[\mathscr{U}_{\mathrm{k}}\right]$ in Theorem 4.17 is $\mathfrak{H}_{2 e^{-}}$equivariant. From Lemmas 4.15 and 5.1 we deduce that there is a $\mathfrak{g}_{e} \times \mathfrak{H}_{2 e}$-equivariant isomorphism given by

$$
\left[\mathscr{U}_{\mathrm{k}}\right] \stackrel{\sim}{\rightarrow} \mathbf{F}(1)_{e, o}, \quad\left[V_{\lambda}\right] \mapsto(-1)^{a(\lambda)}|\lambda, 1\rangle_{e, o}
$$

Remark 5.2. Set $A(\lambda)=m(m-1) / 2-a\left(\lambda^{*}\right)$ with $m=|\lambda|$. By [17, lem. 4.2] we have $(-1)^{A(\lambda)}=\varepsilon_{\lambda}$ where $\varepsilon_{\lambda}$ was defined in 4.3 .1 . From this we can check that

$$
\lambda_{[2]}=\nu_{[2]} \Rightarrow(-1)^{a(\lambda)} \varepsilon_{\lambda}=(-1)^{a(\nu)} \varepsilon_{\nu} .
$$

Hence, the endomorphism of $\left[\mathscr{U}_{\mathrm{k}}\right]$ such that $\left[V_{\lambda}\right] \mapsto(-1)^{a(\lambda)} \varepsilon_{\lambda}\left[V_{\lambda}\right]$ commutes with the action of $\mathfrak{g}_{e}$ and $\mathfrak{H}_{2 e}$. This shows that we could have used the sign $\varepsilon_{\lambda}$ instead of $(-1)^{a(\lambda)}$ in the isomorphism (5.3).

\subsection{The modular Harish-Chandra series of $G L_{m}$.}

Recall that $G L_{n}:=\mathrm{GL}_{n}\left(q^{2}\right)$ denotes the finite general linear group over a finite field with $q^{2}$ elements.

5.2.1. The unipotent modules of $G L_{n}$. The set of unipotent characters of $K G L_{n}$ is

$$
\operatorname{Unip}\left(K G L_{n}\right)=\left\{L_{\lambda} \mid \lambda \in \mathscr{P}_{n}\right\}
$$

where

$$
L_{\lambda}=\left|\mathfrak{S}_{n}\right|^{-1} \sum_{w \in \mathfrak{S}_{n}} \phi_{\lambda}(w) R_{\mathbf{T}_{w}}^{\mathbf{G} \mathbf{L}_{n}}(1)
$$

and $\mathbf{T}_{w}$ is an $F_{q^{2}}$-stable maximal torus of $\mathbf{G L}_{n}$ of cycle-type $\rho(w)$, with the convention that $\mathbf{T}_{1}=\mathbf{T}$ is the split torus of diagonal matrices. There is a unique labeling of the simple unipotent modules of $\mathrm{k} G L_{n}$ given by

$$
\operatorname{Unip}\left(\mathrm{k} G L_{n}\right)=\left\{S_{\lambda} \mid \lambda \in \mathscr{P}_{n}\right\}
$$

where

$$
d_{\mathscr{O} G L_{n}}\left(\left[L_{\lambda}\right]\right)=\left[S_{\lambda}\right] \text { modulo } \bigoplus_{\mu>\lambda} \mathbb{Z}\left[S_{\mu}\right]
$$

As in the case of unitary groups (see Proposition 4.4), there exists a unique indecomposable unipotent $\mathscr{O} G L_{n}$-lattice $\widetilde{W}_{\lambda}$ with character $L_{\lambda}$ and such that $W_{\lambda}:=k \widetilde{W}_{\lambda}$ is indecomposable with simple head $S_{\lambda}$. 
5.2.2. The modular Steinberg character and Harish-Chandra series. First, let us introduce the following notation. Given non-negative integers $m_{j}$ with $j \geqslant-1$, we will say that a partition $\lambda$ of $m$ is cuspidal of type $\left(m_{j}\right)$ if $\lambda$ is of the following form

$$
\lambda=\left(1^{\left(m_{-1}\right)}, e^{\left(m_{0}\right)},(e \ell)^{\left(m_{1}\right)},\left(e \ell^{2}\right)^{\left(m_{2}\right)}, \ldots\right)
$$

in exponential notation.

For a partition $\lambda$ as above, we consider the Levi subgroup of $\mathbf{G L}_{m}$ given by

$$
\mathbf{G L}_{\lambda}=\left(\mathbf{G L}_{1}\right)^{m_{-1}} \times \prod_{j \geqslant 0}\left(\mathbf{G L}_{e \ell^{j}}\right)^{m_{j}} .
$$

Recall that $G L_{\lambda}=\mathbf{G L}_{\lambda}\left(q^{2}\right)$. The unipotent module $S_{\left(1^{m}\right)}$ is the head of $W_{\left(1^{n}\right)}$. Since $W_{\left(1^{n}\right)}$ is a modular reduction of the Steinberg character $L_{\left(1^{m}\right)}$ of $K G L_{m}$, we call $S_{\left(1^{m}\right)}$ the modular Steinberg representation of $\mathrm{k} G L_{m}$ and we write $S t_{m}=S_{\left(1^{m}\right)}$. Let $S t_{\lambda}$ be the $\mathrm{k} G L_{\lambda}$-module given by

$$
S t_{\lambda}=\left(S t_{1}\right)^{\otimes m_{-1}} \otimes \bigotimes_{j \geqslant 0}\left(S t_{e \ell^{j}}\right)^{\otimes m_{j}}
$$

The following result is due to Dipper, James and Dipper-Du [16], see also [8, sec. 19].

\section{Proposition 5.3.}

(a) The cuspidal pairs of $\mathrm{k} G L_{m}$ are the $G L_{m}$-conjugates of the pairs $\left(G L_{\lambda}, S t_{\lambda}\right)$ where $\lambda$ runs over the set of all cuspidal partitions of $m$.

(c) If $\lambda$ is cuspidal of type $\left(m_{j}\right)$, then we have a $\mathrm{k}$-algebra isomorphism

$$
\mathscr{H}\left(\mathrm{k} G L_{m}, S t_{\lambda}\right) \simeq \mathbf{H}_{\mathrm{k}, m_{-1}}^{1 ; q^{2}} \otimes \bigotimes_{j \geqslant 0} \mathbf{H}_{\mathrm{k}, m_{j}}^{1 ; 1}
$$

Recall that here $\mathbf{H}_{\mathrm{k}, m}^{1 ; u}$ denotes the Hecke algebra of type $\mathfrak{S}_{m}$ over k with parameter $u$. In particular, we have $\mathbf{H}_{\mathrm{k}, m}^{1 ; 1}=\mathrm{kS}_{m}$.

\subsection{The Heisenberg functors.}

For $R=K$ or k we call the category of unipotent $R G L$-modules the category

$$
\mathscr{V}_{R}=\bigoplus_{m \in \mathbb{N}} R G L_{m} \text {-umod. }
$$

Recall that the vector space $\Lambda$ is equipped with the bilinear form $\langle\bullet, \bullet\rangle_{\boldsymbol{\Lambda}}$. It induces a bilinear form $\langle\bullet, \bullet\rangle_{\mathscr{V}}$ on $\left[\mathscr{V}_{\mathrm{k}}\right]$ via the isomorphism of vector spaces

$$
\left[\mathscr{V}_{\mathrm{k}}\right] \stackrel{\sim}{\rightarrow} \Lambda, \quad\left[W_{\lambda}\right] \mapsto \phi_{\lambda} .
$$


Now, let us come back to the category $\mathscr{U}_{\mathrm{k}}$ of unipotent modules of the unitary group. Let $\langle\bullet, \bullet\rangle_{\mathscr{U}}$ be the unique bilinear form on $\left[\mathscr{U}_{\mathrm{k}}\right]$ such that the map (5.3) is an isometry.

Given $n, r, m>0$ such that $n=r+2 m$, we write $L_{r, m} \simeq G_{r} \times G L_{m}$. Given a unipotent $R G L_{m}$-module $X$ we consider the following functors

$$
\begin{aligned}
& B_{X}: R G_{r} \text {-umod } \rightarrow R G_{n} \text {-umod, } M \mapsto R_{L_{r, m}}^{G_{n}}(M \otimes X), \\
& B_{X}^{*}: R G_{n} \text {-umod } \rightarrow R G_{r} \text {-umod, } M \mapsto \operatorname{Hom}_{R G L_{m}}\left(X,{ }^{*} R_{L_{r, m}}^{G_{n}}(M)\right) .
\end{aligned}
$$

The functor $B_{X}^{*}$ is right adjoint to $B_{X}$. In the particular case where $m=1$ and $X=R$ is the trivial module, we recover the functors $F, E$ defined in 84.2 . The functor $B_{X}$ is exact, hence it induces a $\mathbb{C}$-linear endomorphism $\left[B_{X}\right]$ of $\left[\mathscr{U}_{\mathrm{k}}\right]$. However, the functor $B_{X}^{*}$ is not exact and the module $X$ may not have a finite projective dimension in $\mathrm{k} G L_{m}$-umod. Hence, the right derived functor of $B_{X}^{*}$ may not yield any endomorphism of $\left[\mathscr{U}_{\mathrm{k}}\right]$. Nevertheless we can avoid this problem by defining the $\mathbb{C}$-linear map

$$
\left[B_{X}^{*}\right]=\left\langle[X],\left[{ }^{*} R_{L_{r, m}}^{G_{n}}\right](\bullet)\right\rangle_{\mathscr{V}}:\left[\mathrm{k} G_{n} \text {-umod }\right] \rightarrow\left[\mathrm{k} G_{r} \text {-umod }\right] .
$$

The Harish-Chandra induction relative to the subgroups $G L_{n_{1}} \times G L_{n_{2}}$ of $G L_{n}$ for $n_{1}+n_{2}=n$ yields a bifunctor $\bullet \odot \bullet: \mathscr{V}_{R} \otimes \mathscr{V}_{R} \rightarrow \mathscr{V}_{R}$.

Proposition 5.4. Let $X, X^{\prime}$ be objects in $\mathscr{V}_{\mathrm{k}}$.

(a) There is an isomorphism of functors $B_{X} B_{X^{\prime}} \simeq B_{X \odot X^{\prime}} \simeq B_{X^{\prime}} B_{X}$.

(b) There is an equality of linear maps $\left[B_{X}^{*}\right] \circ\left[B_{X^{\prime}}^{*}\right]=\left[B_{X^{*} \odot X^{\prime}}^{*}\right]=\left[B_{X^{\prime}}^{*}\right] \circ\left[B_{X}^{*}\right]$.

(c) The linear maps $\left[B_{X}\right],\left[B_{X}^{*}\right]$ are adjoint relatively to the bilinear form on $\left[\mathscr{U}_{\mathrm{k}}\right]$.

(d) If $M \in \mathscr{U}_{\mathrm{k}}$ is cuspidal, then $\left[B_{X}^{*}\right]([M])=0$.

\subsection{The categorification of the Heisenberg action on $\left[\mathscr{U}_{\mathrm{k}}\right]$.}

Recall that $e$ is odd. The representation of $\mathfrak{H}_{2 e}$ on $\left[\mathscr{U}_{\mathrm{k}}\right]$ in $\$ 5.1 .3$ is determined by linear operators $b_{n}, b_{n}^{*}$ with $n$ a positive multiple of $2 e$ satisfying the relations $\left[b_{n}, b_{m}\right]=\left[b_{n}^{*}, b_{m}^{*}\right]=0$ and $\left[b_{n}, b_{m}^{*}\right]=-n \delta_{n, m}$ Id. In this section we will re-interpret some of these operators in representation theoretic terms using the category $\mathscr{U}_{\mathrm{k}}$.

Now, fix a positive integer $m$ and a cuspidal partition $\lambda$ of $m$. Consider the module

$$
X_{\lambda}=R_{G L_{\lambda}}^{G L_{m}}\left(S t_{\lambda}\right)=R_{G L_{\lambda}}^{G L_{m}}\left(\left(S t_{1}\right)^{\otimes m_{-1}} \otimes \bigotimes_{j \geqslant 0}\left(S t_{e \ell^{j}}\right)^{\otimes m_{j}}\right) .
$$

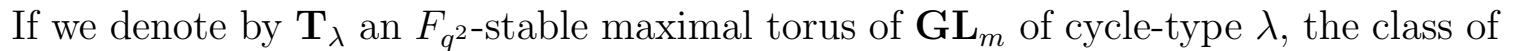
$X_{\lambda}$ coincides with the Deligne-Lusztig character induced from $\mathbf{T}_{\lambda}$.

Lemma 5.5. If $\lambda$ is a cuspidal partition of $m$ then $\left[X_{\lambda}\right]=R_{\mathbf{T}_{\lambda}}^{\mathbf{G} \mathbf{L}_{m}}(1)$ in $\left[\mathscr{U}_{\mathrm{k}}\right]$. 
Proof. The finite group $T_{\lambda}=\mathbf{T}_{\lambda}\left(q^{2}\right)$ is isomorphic to $\mathbf{G L}_{1}\left(q^{2}\right)^{m_{-1}} \times \prod_{j \geqslant 0} \mathbf{G L}_{1}\left(q^{2 e \ell^{j}}\right)^{m_{j}}$. By [8, thm. 19.18], for each integer $j \geqslant 0$ there is a character $\theta$ of $K \mathbf{G L}_{1}\left(q^{2 e \ell^{j}}\right)$ of order a power of $\ell$ such that we have

$$
R_{\mathbf{T}_{\left(e \ell^{j}\right)}}^{\mathbf{G L}_{e \ell j}}(1)=d_{\mathscr{O} G L} R_{\mathbf{T}_{\left(e \ell^{j}\right)}}^{\mathbf{G} \mathbf{L}_{e j}}([\theta])=(-1)^{e \ell^{j}-1}\left[S t_{e \ell^{j}}\right]=\left[S t_{e \ell^{j}}\right] .
$$

We deduce that $R_{\mathbf{T}_{\lambda}}^{\mathbf{G L}_{\lambda}}(1)=\left[S t_{\lambda}\right]$. By transitivity of the Lusztig induction we get

$$
\left[X_{\lambda}\right]=R_{G L_{\lambda}}^{G L_{m}}\left(\left[S t_{\lambda}\right]\right)=R_{\mathbf{T}_{\lambda}}^{\mathbf{G L}}(1) .
$$

Given a cuspidal partition $\lambda$, we set $B_{\lambda}=B_{X_{\lambda}}$. Note that the endofunctor $F$ of $\mathscr{U}_{\mathrm{k}}$ is equal to $B_{1}$. The functor $B_{\lambda}^{*}$ is not well-defined, but we may still consider the linear endomorphism $\left[B_{\lambda}^{*}\right]$ of $\left[\mathscr{U}_{\mathrm{k}}\right]$. They are related to the Heisenberg operators considered earlier by the following proposition (which will not be used in the rest of the paper).

Proposition 5.6. Assume that $\ell \neq 2$. Let $\lambda$ be a cuspidal partition of $m$ of type $\left(m_{j}\right)$ with $m_{-1}=0$. Then, we have the equalities $b_{2 \lambda}=\left[B_{\lambda}\right]$ and $b_{2 \lambda}^{*}=\left[B_{\lambda}^{*}\right]$ in $\operatorname{End}\left(\left[\mathscr{U}_{\mathrm{k}}\right]\right)$.

To prove the proposition we need more material. Fix a positive integer $a$. We abbreviate $G L_{m}^{(a)}=\mathbf{G L}_{m}\left(q^{2 a}\right)$ and we consider the category

$$
\mathscr{V}_{R}^{(a)}:=\bigoplus_{m \in \mathbb{N}} R G L_{m}^{(a)} \text {-umod. }
$$

First, we view $G L_{m}^{(a)}$ as the group of $\mathbb{F}_{q^{2 a}}$-rational points of the rational group $\left(\mathbf{G L}_{m}, F_{q^{2 a}}\right)$. Unipotent irreducible modules of $K G L_{m}$ and $K G L_{m}^{(a)}$ are both parametrized by partitions of $m$. Therefore there is an isomorphism $\left[\mathscr{V}_{\mathrm{k}}\right] \stackrel{\sim}{\rightarrow}\left[\mathscr{V}_{\mathrm{k}}^{(a)}\right]$ sending $\left[W_{\mu}\right]$ to $\left[W_{\mu}^{(a)}\right]$ for any partition $\mu$ of $m$. More generally we will denote by $\left[V^{(a)}\right]$ the image of $[V]$ under this map. By composition we obtain $\mathbb{C}$-linear isomorphisms given by

$$
\left[\mathscr{V}_{K}^{(a)}\right] \stackrel{\sim}{\rightarrow}\left[\mathscr{V}_{\mathrm{k}}^{(a)}\right] \stackrel{\sim}{\rightarrow} \Lambda, \quad\left[L_{\mu}^{(a)}\right] \mapsto\left[W_{\mu}^{(a)}\right] \mapsto \phi_{\mu}
$$

Next, we view $G L_{m}^{(a)}$ as the group of rational points of $\mathbf{G L}_{m}^{(a)}=\left(\left(\mathbf{G L}_{m}\right)^{a}, \sigma_{a} F_{q^{2}}\right)$, where $\sigma_{a}$ is the automorphism of $\left(\mathbf{G L}_{m}\right)^{a}$ such that $\left(g_{1}, g_{2}, \ldots, g_{a}\right) \mapsto\left(g_{2}, \ldots, g_{a}, g_{1}\right)$. Note that $\mathbf{G L}_{m}^{(a)}$ is a Levi subgroup of $\mathbf{G L}_{a m}=\left(\mathbf{G L}_{a m}, F_{q^{2}}\right)$, but the rational structures are different. The Lusztig induction and restriction yield $\mathbb{C}$-linear maps

$$
\begin{aligned}
R_{\mathbf{G L}_{m}^{(a)}}^{\mathbf{G} \mathbf{L}_{a m}}:\left[\mathrm{k} G L_{m}^{(a)} \text {-umod }\right] & \rightarrow\left[\mathrm{k} G L_{a m} \text {-umod }\right], \\
{ }^{*} R_{\mathbf{G L}_{m}^{(a)}}^{\mathbf{G} \mathbf{L}_{a m}} & :\left[\mathrm{k} G L_{a m} \text {-umod] } \rightarrow\left[\mathrm{k} G L_{m}^{(a)} \text {-umod }\right] .\right.
\end{aligned}
$$


Let $\mathscr{F}^{a}$ be the $\mathbb{C}$-linear map $\boldsymbol{\Lambda} \rightarrow \boldsymbol{\Lambda}$ such that $\mathscr{F}^{a}\left(z_{\lambda} c_{\lambda}\right)=z_{a \lambda} c_{a \lambda}$ for each partition $\lambda$. Let $\mathscr{F}_{a}$ be the transposed map relative to the bilinear form on $\Lambda$. We have $\mathscr{F}_{a}\left(c_{\lambda}\right)=c_{\mu}$ if $\lambda=a \mu$ and $\mathscr{F}_{a}\left(c_{\lambda}\right)=0$ if $\lambda \neq a \mu$ for all $\mu$.

\section{Lemma 5.7.}

(a) The isomorphism (5.7) identifies the maps $R_{\mathbf{G L}_{m}^{(a)}}^{\mathbf{G L}_{a m}}$ and ${ }^{*} R_{\mathbf{G L}_{m}^{(a)}}^{\mathbf{G L}_{a m}}$ with the endomorphisms $\mathscr{F}^{a}$ and $\mathscr{F}_{a}$ of $\Lambda$.

(b) The isomorphism (5.6) identifies the maps $R_{G L_{m} \times G L_{n}}^{G L_{m+n}}$ and ${ }^{*} R_{G L_{m} \times G L_{n}}^{G L_{m+n}}$ with the maps $\operatorname{Ind}_{m, n}^{m+n}$ and $\operatorname{Res}_{m, n}^{m+n}$ between $\Lambda \otimes \Lambda$ and $\Lambda$.

(c) If $n=r+2 m$, the isomorphism $\left[\mathscr{U}_{\mathrm{k}}\right] \stackrel{\sim}{\rightarrow} \boldsymbol{\Lambda}$ sending $\varepsilon_{\lambda}\left[V_{\lambda}\right]$ to $\phi_{\lambda}$ and the isomorphism $\left[\mathscr{V}_{\mathrm{k}}\right] \stackrel{\sim}{\rightarrow} \Lambda$ in (5.7) identify the maps $R_{L_{r, m}}^{G_{n}}$ and ${ }^{*} R_{L_{r, m}}^{G_{n}}$ with the maps $\operatorname{Ind}_{r, 2 m}^{n}\left(\bullet \otimes \mathscr{F}^{2}(\bullet)\right)$ and $\left(1 \otimes \mathscr{F}_{2}\right) \operatorname{Res}_{r, 2 m}^{n}$ between $\boldsymbol{\Lambda} \otimes \boldsymbol{\Lambda}$ and $\boldsymbol{\Lambda}$.

Proof. The proof of the lemma is standard, see, e.g., [63, prop. 6.1] or [4, sec. 3.A]. Let us give some details.

First, we prove part (a). We abbreviate $n=a m$. Consider the rational groups $\left(\mathbf{G L}_{n}, F_{q^{2}}\right)$ and $\left(\left(\mathbf{G L}_{m}\right)^{a}, \sigma_{a} F_{q^{2}}\right)$. We will denote them by $\mathbf{G}$ and $\mathbf{G}^{(a)}$ respectively. The Weyl groups of $\mathbf{G}$ and $G$ are identified with $\mathfrak{S}_{n}$. The Weyl group of $\mathbf{G}^{(a)}$ is $\left(\mathfrak{S}_{m}\right)^{a}$. Let $\sigma_{a}$ denote also the permutation in $\mathfrak{S}_{n}$ of cycle-type $\left(a^{m}\right)$ which normalizes the subgroup $\left(\mathfrak{S}_{m}\right)^{a}$ and induces the automorphism such that $\left(w_{1}, w_{2}, \ldots, w_{a}\right) \mapsto$ $\left(w_{2}, \ldots, w_{a}, w_{1}\right)$. The action of the Frobenius homomorphism on the Weyl group of $\mathbf{G}^{(a)}$ is given by the conjugation by $\sigma_{a}$. The Weyl group of $G^{(a)}$ is $\mathfrak{S}_{m}$.

By (5.5) we have

$$
\left[L_{\lambda}\right]=\sum_{\nu \vdash n}\left\langle\phi_{\lambda}, c_{\nu}\right\rangle_{\Lambda} R_{\mathbf{T}_{\nu}}^{\mathbf{G}}(1)
$$

Hence, the isometry $\left[\mathscr{V}_{\mathrm{k}}\right] \stackrel{\sim}{\rightarrow} \boldsymbol{\Lambda}$ such that $\left[L_{\lambda}\right] \mapsto \phi_{\lambda}$ maps the virtual module $R_{\mathbf{T}_{\lambda}}^{\mathbf{G}}(1)$ to $z_{\lambda} c_{\lambda}$ for each partition $\lambda$ of $n$. Similarly, the isometry $\left[\mathscr{V}_{\mathrm{k}}^{(a)}\right] \rightarrow \Lambda$ in (5.7) maps $\left[L_{\mu}^{(a)}\right]$ to $\phi_{\mu}$ for each partition $\mu$ of $m$, hence it maps the virtual module $\left(R_{\mathbf{T}_{\mu}}^{\mathbf{G L}_{m}}(1)\right)^{(a)}$ to $z_{\mu} c_{\mu}$.

With the notation from Remark 3.2 for Lusztig induction, we have

$$
\begin{aligned}
& \left(R_{\mathbf{T}_{\mu}}^{\mathbf{G} \mathbf{L}_{m}}(1)\right)^{(a)}=\left(R_{\mathbf{T}_{\mu}, F_{q^{2}}}^{\mathbf{G} \mathbf{L}_{m}, F_{q^{2}}}(1)\right)^{(a)} \\
& =R_{\mathbf{T}_{\mu}, F_{q^{2 a}}}^{\mathbf{G L} \mathbf{L}_{m}, F_{2 a}} \\
& =R_{\mathbf{T}_{w}, \sigma_{a} F_{q^{2}}}^{\left(\mathbf{G L}_{m}\right)^{a}, \sigma_{a} F_{q^{2}}}(1),
\end{aligned}
$$


where $w=\left(w_{1}, w_{2}, \ldots, w_{a}\right)$ is any element in $\left(\mathfrak{S}_{m}\right)^{a}$ such that $w_{1} w_{2} \cdots w_{a}$ is of cycle-type $\mu$. By transitivity of Lusztig induction we deduce that

$$
R_{\mathbf{G}^{(a)}}^{\mathbf{G}}\left(R_{\mathbf{T}_{\mu}}^{\mathbf{G} \mathbf{L}_{m}}(1)\right)^{(a)}=R_{\mathbf{T}_{w \sigma_{a}}}^{\mathbf{G}}(1)=R_{\mathbf{T}_{a \mu}}^{\mathbf{G}}(1),
$$

because $w \sigma_{a}$ is of cycle-type $a \mu$. Therefore, up to the isometries above, the map

$$
R_{\mathbf{G}^{(a)}}^{\mathbf{G}}:\left[\mathscr{V}_{K}^{(a)}\right] \rightarrow\left[\mathscr{V}_{K}\right]
$$

is identified with the linear endomorphism $\mathscr{F}^{a}$ of $\Lambda$. This proves the first identity in part (a). The second one follows by adjunction.

Now, we prove part (b). Consider the $\mathbb{F}_{q^{2}}$-rational group $\left(\mathbf{G L}_{m+n}, F_{q^{2}}\right)$ with its $F_{q^{2}}$-stable Levi subgroup $\left(\mathbf{G L}_{m} \times \mathbf{G L}_{n}, F_{q^{2}}\right)$. The isometry $\left[K G L_{m}\right.$-umod $] \rightarrow$ $\mathbb{C} \operatorname{Irr}\left(K \mathfrak{S}_{m}\right)$ in (5.6) maps the virtual module $R_{\mathbf{T}_{\mu}}^{\mathbf{G L}_{m}}(1)$ to $z_{\mu} c_{\mu}$ for each partition $\mu$ of $m$. By the transitivity of Lusztig induction we get

$$
R_{G L_{m} \times G L_{n}}^{G L_{m+n}} R_{\mathbf{T}_{\mu} \times \mathbf{T}_{\lambda}}^{\mathbf{G L}_{m} \times \mathbf{G L}_{n}}(1)=R_{\mathbf{T}_{\mu} \times \mathbf{T}_{\lambda}}^{\mathbf{G \mathbf { L } _ { m + n }}}(1) .
$$

This proves the first identity in part (b). The second one follows by adjunction.

It remains to prove part (c). Consider the $\mathbb{F}_{q}$-rational group $\left(\mathbf{G L}_{n}, F\right)$ with its $F$ stable Levi subgroup $\mathbf{L}_{r, m}$. We will abbreviate $\mathbf{G}_{n}=\left(\mathbf{G L}_{n}, F\right)$ and we will identify the rational groups $\left(\mathbf{L}_{r, m}, F\right)$ and $\left(\mathbf{G L}_{r} \times\left(\mathbf{G L}_{m}\right)^{2},\left(F, \sigma_{2} F_{q}\right)\right)$, where $\sigma_{2}$ is an element of $\mathfrak{S}_{2 m}$ of cycle-type $\left(2^{m}\right)$. Let $w_{n}$ denote the longest element in $\mathfrak{S}_{n}$. The Weyl groups of $\mathbf{G L}_{n}$ and $G_{n}$ are identified with $\mathfrak{S}_{n}$ and $W_{n}=C_{\mathfrak{S}_{n}}\left(w_{n}\right)$. The Weyl group of $\mathbf{L}_{r, m}$ is identified with $\mathfrak{S}_{r} \times\left(\mathfrak{S}_{m}\right)^{2}$ with the action of the Frobenius endomorphism $F$ given by conjugation by $\left(w_{r}, \sigma_{2}\right)$. Here, we embed $\mathfrak{S}_{r} \times\left(\mathfrak{S}_{m}\right)^{2}$ into $\mathfrak{S}_{n}$ via the map $(u, v) \mapsto\left(w_{m} v_{1} w_{m}, u, v_{2}\right)$ if $v=\left(v_{1}, v_{2}\right)$ with $v_{1}, v_{2} \in \mathfrak{S}_{m}$. The Weyl group of $L_{r, m}$ is identified with $W_{r} \times \mathfrak{S}_{m}$.

From (4.2) we deduce that the isometry $\left[\mathscr{U}_{K}\right] \stackrel{\sim}{\rightarrow} \Lambda$ such that $\chi_{\lambda} \mapsto \phi_{\lambda}$ maps the virtual module $R_{\mathbf{T}_{\lambda}}^{\mathbf{G}_{r}}(1)$ to $z_{\lambda} c_{\lambda}$ for each partition $\lambda$ of $r$. In the proof of (a) we already observed that the isometry $\left[\mathscr{V}_{K}\right] \stackrel{\sim}{\rightarrow} \Lambda$ in (5.7) maps the virtual module $R_{\mathbf{T}_{\mu}, F_{q^{2}}}^{\mathbf{G} \mathbf{L}_{m}, F_{q^{2}}}(1)=R_{\mathbf{T}_{\mu} \times \mathbf{T}_{1}, \sigma_{2} F_{q}}^{\left(\mathbf{G L}_{m}\right)^{2}, \sigma_{2} F_{q}}(1)$ to $z_{\mu} c_{\mu}$ for each partition $\mu$ of $m$. By transitivity of Lusztig induction we get

$$
R_{\mathbf{L}_{r, m}, F}^{\mathbf{G L} \mathbf{L}_{n}, F}\left(R_{\mathbf{T}_{\lambda} \times \mathbf{T}_{\mu} \times \mathbf{T}_{1},\left(F, \sigma_{2} F_{q}\right)}^{\mathbf{G L} \mathbf{G}_{2} \times\left(\mathbf{G L}_{m}\right)^{2},\left(F, \sigma_{2} F_{q}\right)}(1)\right)=R_{\mathbf{T}_{\mu} \times \mathbf{T}_{\lambda} \times \mathbf{T}_{1}, F}^{\mathbf{G \mathbf { L } _ { n } , F}}(1) .
$$

We deduce that the map $R_{L_{r, m}}^{G_{n}}$ is identified with the map $\mathbb{C} \operatorname{Irr}\left(\mathfrak{S}_{r}\right) \otimes \mathbb{C} \operatorname{Irr}\left(\mathfrak{S}_{m}\right) \rightarrow$ $\mathbb{C} \operatorname{Irr}\left(\mathfrak{S}_{n}\right)$ given by $\operatorname{Ind}_{r, 2 m}^{n}\left(\bullet \otimes \mathscr{F}^{2}(\bullet)\right)$. This proves the first identity in part (c). The second one follows by adjunction. 
Proof of Proposition 5.6. Let $n=r+2 m$. Under the isomorphism (5.6) and the isomorphism $\left[\mathscr{U}_{\mathrm{k}}\right] \stackrel{\sim}{\rightarrow} \Lambda$ given by $\varepsilon_{\lambda}\left[V_{\lambda}\right] \mapsto \phi_{\lambda}$, Lemma 5.7 identifies the maps $R_{L_{r, m}}^{G_{n}}$ and ${ }^{*} R_{L_{r, m}}^{G_{n}}$ between $\left[\mathscr{U}_{\mathrm{k}} \otimes \mathscr{V}_{\mathrm{k}}\right]$ and $\left[\mathscr{V}_{\mathrm{k}}\right]$ with the $\mathbb{C}$-linear maps $\boldsymbol{\Lambda} \otimes \boldsymbol{\Lambda} \rightarrow \boldsymbol{\Lambda}$ and $\Lambda \rightarrow \Lambda \otimes \Lambda$ given by

$$
\phi_{\nu} \otimes \phi_{\lambda} \mapsto \operatorname{Ind}_{r, 2 m}^{n}\left(\phi_{\nu} \otimes \mathscr{F}^{2}\left(\phi_{\lambda}\right)\right), \quad \phi_{\nu} \mapsto\left(1 \otimes \mathscr{F}_{2}\right) \operatorname{Res}_{r, 2 m}^{n}\left(\phi_{\nu}\right) .
$$

Now, assume that $\lambda$ is cuspidal of type $\left(m_{j}\right)$. By Lemmas 5.5, 5.7 the class of $X_{\lambda}$ in $\left[\mathscr{V}_{\mathrm{k}}\right]$ is identified with the element $z_{\lambda} c_{\lambda} \in \boldsymbol{\Lambda}$. From (5.1) we deduce that the operator $\left[B_{\lambda}\right]=\bigoplus_{r} R_{L_{r, m}}^{G_{r+2 m}}\left(\bullet \otimes\left[X_{\lambda}\right]\right)$ on $\left[\mathscr{U}_{\mathrm{k}}\right]$ is identified with the operators $b_{2 \lambda}$ on $\boldsymbol{\Lambda}$. Hence, the proposition follows from Lemma 5.1 and Remark 5.2.

\subsection{Cuspidal modules and highest weight vectors.}

By Proposition 4.20, the set of isomorphism classes of weakly cuspidal modules in $\mathscr{U}_{\mathrm{k}}$ is a basis of the space $\left[\mathscr{U}_{\mathrm{k}}\right]^{0}$ of all elements in $\left[\mathscr{U}_{\mathrm{k}}\right]$ which are killed by the map $\left[E_{i}\right]$ for each $i \in I_{e}$. We define

$$
\left[\mathscr{U}_{\mathrm{k}}\right]^{\mathrm{hw}}=\left\{x \in\left[\mathscr{U}_{\mathrm{k}}\right]^{0} \mid b_{n}^{*}(x)=0, \forall n \in 2 e \mathbb{Z}_{>0}\right\} .
$$

Then, we have the following inclusion.

Lemma 5.8. $\left\{[D] \mid D \in \operatorname{Irr}\left(\mathscr{U}_{\mathrm{k}}\right)\right.$ is cuspidal $\} \subseteq\left[\mathscr{U}_{\mathrm{k}}\right]^{\mathrm{hw}}$.

Proof. For any partition $\lambda$ we set

$$
a_{2 \lambda}=\sum_{\nu} \chi_{\nu}^{\lambda} z_{\nu}^{-1} b_{2 \nu}, \quad a_{2 \lambda}^{*}=\sum_{\nu} \chi_{\nu}^{\lambda} z_{\nu}^{-1} b_{2 \nu}^{*}
$$

where $\chi_{\nu}^{\lambda}=\left\langle\phi_{\lambda}, z_{\nu} c_{\nu}\right\rangle_{\Lambda}$ is the value of the irreducible character $\phi_{\lambda}$ on a permutation of cycle-type $\nu$. Since the $\mathscr{P} \times \mathscr{P}$-matrix with entries $\chi_{\nu}^{\lambda}$ is invertible, we have

$$
\left[\mathscr{U}_{\mathrm{k}}\right]^{\mathrm{hw}} \supseteq\left\{x \in\left[\mathscr{U}_{\mathrm{k}}\right]^{0} \mid a_{2 \lambda}^{*}(x)=0, \forall \lambda \in \mathscr{P}\right\} .
$$

Now, for any partition $\lambda$ we set $A_{\lambda}=B_{W_{\lambda}}$ and $\left[A_{\lambda}^{*}\right]=\left[B_{W_{\lambda}}^{*}\right]$. Under the isomorphism $\left[\mathscr{U}_{\mathrm{k}}\right] \simeq \Lambda$, the operator $\left[A_{\lambda}\right]$ on $\left[\mathscr{U}_{\mathrm{k}}\right]$ is identified by $(\underline{5.8})$ with the operator $\bigoplus_{r} \operatorname{Ind}_{r, 2 m}^{r+2 m}\left(\bullet \otimes \mathscr{F}^{2}\left(\phi_{\lambda}\right)\right)$ on $\Lambda$. Since $\phi_{\lambda}=\sum_{\nu} \chi_{\nu}^{\lambda} c_{\nu}$, the formula (5.1) yields

$$
\bigoplus_{r} \operatorname{Ind}_{r, 2 m}^{r+2 m}\left(\bullet \otimes \mathscr{F}^{2}\left(\phi_{\lambda}\right)\right)=\sum_{\nu} \chi_{\nu}^{\lambda} z_{\nu}^{-1} b_{2 \nu}
$$

We deduce that we have $a_{2 \lambda}=\left[A_{\lambda}\right]$ and $a_{2 \lambda}^{*}=\left[A_{\lambda}^{*}\right]$ in $\operatorname{End}\left(\left[\mathscr{U}_{\mathrm{k}}\right]\right)$. Now, to prove the lemma it is enough to prove that $a_{2 \lambda}^{*}([D])=0$ for all partition $\lambda$ and all cuspidal module $D$ in $\operatorname{Irr}\left(\mathscr{U}_{\mathrm{k}}\right)$. This follows from Proposition 5.4 . 
5.5.1. The parameters of the ramified Hecke algebras. It is not obvious that the vector space $\left[\mathscr{U}_{\mathrm{k}}\right]^{\mathrm{hw}}$ is spanned by classes of irreducible unipotent modules. Our goal is to determine precisely the cuspidal modules. Let us first recall the following basic facts.

\section{Lemma 5.9.}

(a) Any unipotent cuspidal pair of $\mathrm{k} G_{n}$ is $G_{n}$-conjugate to a pair $\left(G_{r} \times G L_{\lambda}, D_{\nu} \otimes\right.$ $\left.S t_{\lambda}\right)$ where $n=r+2 m, \lambda$ is a cuspidal partition of $m$ and $D_{\nu}$ is a unipotent cuspidal $\mathrm{k} G_{r}$-module.

(b) Given a cuspidal pair $\left(G_{r} \times G L_{\lambda}, D_{\nu} \otimes S t_{\lambda}\right)$ with $\lambda$ of type $\left(m_{j}\right)$, the ramified Hecke algebra $\mathscr{H}\left(\mathrm{k} G_{n}, D_{\nu} \otimes S t_{\lambda}\right)$ is isomorphic to the $\mathrm{k}$-algebra $\mathbf{H}_{\mathrm{k}, m_{-1}}^{Q_{t}} \otimes$ $\bigotimes_{j \geqslant 0} \mathbf{H}_{\mathrm{k}, m_{j}}^{1,1 ; 1}$, where the integer $t \in \mathbb{N}$ is such that the e-core of $\nu$ is $\Delta_{t}$.

Proof. Part (a) follows from Proposition 5.3. To prove (b), let first observe that by [32, prop. 4.4] we have an algebra isomorphism

$$
\mathscr{H}\left(\mathrm{k} G_{n}, D_{\nu} \otimes S t_{\lambda}\right) \simeq \mathbf{H}_{\mathrm{k}, m_{-1}}^{P_{-1} ; q^{2}} \otimes \bigotimes_{j \geqslant 0} \mathbf{H}_{\mathrm{k}, m_{j}}^{P_{j} ; 1},
$$

where $P_{j}=\left(a_{j}, b_{j}\right)$ is a parameter in $\mathrm{k}^{\times} \times \mathrm{k}^{\times}$for each $j \geqslant-1$. Using the transitivity and faithfulness of induction, see [8, prop. 1.23] for details, we deduce from Corollary 4.23 that $P_{-1}=Q_{t}$ for some $t \geqslant 0$. By [38, prop. 2.3.5] the parameter $P_{j}$ is of the form $\left(a_{j}, a_{j}\right)$ for some element $a_{j} \in \mathrm{k}^{\times}$for each $j \geqslant 0$.

5.5.2. The theorem. We can now formulate the theorem comparing cuspidal modules and highest weight vectors.

Theorem 5.10. The set $\left\{[D] \mid D \in \operatorname{Irr}\left(\mathscr{U}_{\mathrm{k}}\right)\right.$ is cuspidal $\}$ is a basis of $\left[\mathscr{U}_{\mathrm{k}}\right]^{\mathrm{hw}}$.

Proof. First, let us fix some notation that we will use in the whole proof. For every sequence $\left(m_{-1}, m_{0}, m_{1}, \ldots\right)$ of integers $\geqslant 0$ we set $m_{+}=\sum_{j \geqslant 0} m_{j} \ell^{j}, m=m_{-1}+e m_{+}$ and $n=r+2 m$. Let $\lambda$ be the cuspidal partition of $m$ of type $\left(m_{-1}, m_{0}, \ldots\right)$ and $\lambda_{+}$be the cuspidal partition of type $\left(m_{0}, m_{1}, \ldots\right)$. Let $\mathscr{C} \subseteq \mathscr{P}$ be the set of all partitions $\nu$ such that the module $D_{\nu}$ is cuspidal. For each $\nu \in \mathscr{C}$ the $e$-core of $\nu$ is a 2-core $\Delta_{t(\nu)}$. We will abbreviate $\Delta_{\nu}=\Delta_{t(\nu)}$ and $Q_{\nu}=Q_{t(\nu)}$.

Now, we consider the Harish-Chandra series of $\mathrm{k} G_{n}$. The crystal basis $B(\Lambda)$ of the $\mathfrak{g}_{e}$-module $\mathbf{L}(\Lambda)$ is a disjoint union of bases $B(\Lambda)_{\alpha}$ of the weight subspaces $\mathbf{L}(\Lambda)_{\Lambda-\alpha}$ where $\alpha$ runs over $\mathrm{Q}_{e}^{+}$. For each integer $d \geqslant 0$ we set

$$
\mathbf{L}(\Lambda)_{d}=\bigoplus_{\operatorname{ht}(\alpha)=d} \mathbf{L}(\Lambda)_{\Lambda-\alpha}, \quad B(\Lambda)_{d}=\bigsqcup_{\operatorname{ht}(\alpha)=d} B(\Lambda)_{\Lambda-\alpha} .
$$

We first prove the following.

\footnotetext{
${ }^{1}$ The reference [38, prop. 2.3.5] has been indicated to us by G. Hiss.
} 
Claim 5.11. Given $\nu$ a partition, and $m_{-1}, m_{+} \geq 0$, we have a bijection

$$
\bigsqcup_{\lambda} \operatorname{Irr}\left(\mathrm{k} G_{n}, D_{\nu} \otimes S t_{\lambda}\right) \stackrel{1: 1}{\longleftrightarrow} B\left(\Lambda_{Q_{\nu}}\right)_{m_{-1}} \times \mathscr{P}_{m_{+}}
$$

where $\lambda$ runs over all cuspidal partitions with fixed $m_{-1}$ and $m_{+}$.

From Lemma 5.9 and the generalities in Section 3.4, we have a canonical bijection

$$
\operatorname{Irr}\left(\mathrm{k} G_{n}, D_{\nu} \otimes S t_{\lambda}\right) \stackrel{1: 1}{\longleftrightarrow} \operatorname{Irr}\left(\mathbf{H}_{\mathrm{k}, m_{-1}}^{Q_{\nu} ; q^{2}}\right) \times \prod_{j \geqslant 0} \operatorname{Irr}\left(\mathbf{H}_{\mathrm{k}, m_{j}}^{1,1 ; 1}\right) .
$$

By $§ 1.4$, there is a canonical bijection

$$
B\left(\Lambda_{Q_{\nu}}\right)_{m_{-1}} \stackrel{1: 1}{\longleftrightarrow} \operatorname{Irr}\left(\mathbf{H}_{\mathrm{k}, m_{-1}}^{Q_{\nu} ; q^{2}}\right) .
$$

Further, the elements of $\operatorname{Irr}\left(\mathbf{H}_{\mathrm{k}, m_{j}}^{1,1 ; 1}\right)$ are labelled by the $\ell$-restricted partitions of $m_{j}$, see, e.g., [33, prop. 4.6.6]. Hence, the unicity of the $\ell$-adic expansion of partitions yields the following bijection

$$
\mathscr{P}_{m_{+}} \stackrel{1: 1}{\longleftrightarrow} \bigsqcup_{\lambda_{+}} \prod_{j \geqslant 0} \operatorname{Irr}\left(\mathbf{H}_{\mathrm{k}, m_{j}}^{1,1 ; 1}\right)
$$

where $\lambda_{+}=\left(e^{m_{0}},(e \ell)^{m_{1}}, \ldots\right)$ runs over all cuspidal partitions of $m_{+}$with parts divisible by $e$ (see, e.g., [7, lem. 19.26]). The Claim 5.11 follows from (5.9), (5.10) and (5.11).

Now, we consider the complexified Grothendieck group [ $\left.\mathscr{U}_{\mathrm{k}}\right]$. The modular HarishChandra theory yields bijections

$$
\operatorname{Irr}\left(\mathscr{U}_{\mathrm{k}}\right) \stackrel{1: 1}{\longleftrightarrow} \bigsqcup_{n, \nu, \lambda} \operatorname{Irr}\left(\mathrm{k} G_{n}, D_{\nu} \otimes S t_{\lambda}\right) .
$$

Next, we identify $\left[\mathscr{U}_{\mathrm{k}}\right]$ with the $\mathfrak{g}_{e}$-module $\bigoplus_{t} \mathbf{F}\left(Q_{t}\right)$. Fix a basis $B$ of $\left[\mathscr{U}_{\mathrm{k}}\right]^{\text {hw }}$ containing the family $\left\{\left[D_{\nu}\right] \mid \nu \in \mathscr{C}\right\}$. By Corollary 4.23 we can identify the $\mathfrak{g}_{e}^{\prime}$-submodule of $\left[\mathscr{U}_{\mathrm{k}}\right]$ generated by $\left[D_{\nu}\right]$ with $\mathbf{L}\left(\Lambda_{Q_{\nu}}\right)$. We get a canonical isomorphism of $\mathfrak{H}_{2 e} \oplus \mathfrak{g}_{e^{\prime}}^{\prime}$ modules

$$
\left[\mathscr{U}_{\mathrm{k}}\right]=\bigoplus_{\nu \in \mathscr{C}} U\left(\mathfrak{H}_{2 e}\right)\left(\mathbf{L}\left(\Lambda_{Q_{\nu}}\right)\right) \oplus \bigoplus_{b \in B \backslash \mathscr{C}} U\left(\mathfrak{H}_{2 e}\right) U\left(\mathfrak{g}_{e}^{\prime}\right)(b)
$$

For each $n$ let $\left[\mathscr{U}_{\mathrm{k}}\right]_{n}$ be the subspace of $\left[\mathscr{U}_{\mathrm{k}}\right]$ given by

$$
\left[\mathscr{U}_{\mathrm{k}}\right]_{n}=\bigoplus_{\nu, m_{-1}, m_{+}, \gamma} b_{2 e \gamma}\left(\mathbf{L}\left(\Lambda_{Q_{\nu}}\right)_{m_{-1}}\right)
$$


where $\gamma \in \mathscr{P}_{m_{+}}$and $r, m_{-1}, m_{+} \in \mathbb{N}$ are such that $n=r+2 m_{-1}+2 e m_{+}$. Comparing (5.12) and (5.13), we deduce that

$$
\sum_{\nu, \lambda}\left|\operatorname{Irr}\left(\mathrm{k} G_{n}, D_{\nu} \otimes S t_{\lambda}\right)\right| \geqslant \operatorname{dim}\left(\left[\mathscr{U}_{\mathrm{k}}\right]_{n}\right),
$$

and that we have equality for all $n$ if and only if $B=\left\{\left[D_{\nu}\right] \mid \nu \in \mathscr{C}\right\}$.

Now, any non-zero highest-weight $\mathfrak{H}_{2 e}$-module is free as a module over the subalgebra generated by $\left\{b_{2 e n} \mid n \geqslant 1\right\}$. Hence, for each $n$, the Claim 5.11 gives

$$
\sum_{\nu, \lambda}\left|\operatorname{Irr}\left(\mathrm{k} G_{n}, D_{\nu} \otimes S t_{\lambda}\right)\right|=\operatorname{dim}\left(\left[\mathscr{U}_{\mathrm{k}}\right]_{n}\right),
$$

proving the theorem.

Remark 5.12. The theorem implies that

$$
\left\{[D] \mid D \in \operatorname{Irr}\left(\mathscr{U}_{\mathrm{k}}\right) \text { is cuspidal }\right\}=\operatorname{Irr}\left(\mathscr{U}_{\mathrm{k}}\right) \cap\left[\mathscr{U}_{\mathrm{k}}\right]^{\mathrm{hw}} .
$$

5.5.3. Cuspidal modules and FLOTW e-partitions. Recall that in Theorem 4.21 we have given an explicit isomorphism between the crystal basis $B\left(\mathscr{U}_{\mathrm{k}}\right)$, whose underlying set is $\operatorname{Irr}\left(\mathscr{U}_{\mathrm{k}}\right)$, and the abstract crystal $B_{e}$ of the $\mathfrak{g}_{e} \times \mathfrak{H}_{2 e}$-module $\bigoplus_{t} \mathbf{F}\left(Q_{t}\right)_{e}$. Using Theorem 5.10, we deduce that the cuspidal modules in $\operatorname{Irr}\left(\mathscr{U}_{\mathrm{k}}\right)$ corresponds precisely to the elements of $B_{e}$ whose associated elements in the upper global basis $\mathbf{B}_{e}^{\vee}:=\bigsqcup_{t} \mathbf{B}^{\vee}\left(s_{t}\right)$ of $\bigoplus_{t} \mathbf{F}\left(Q_{t}\right)_{e}$, see $\$ 2.3 .2$, are highest weight vectors for the action of both $\mathfrak{g}_{e}$ and $\mathfrak{H}_{2 e}$.

These elements of $B_{e}$ have been computed recently in [35]. To do so, one defines a class of $e$-partitions called FLOTW, see [35, def. 6.22]. Then, the cuspidal modules are related to FLOTW e-partitions as in [35, thm. 7.7].

\section{TyPes B AND C}

This section is devoted to the construction of categorical actions on the category of unipotent representations of the finite groups $\mathrm{SO}_{2 n+1}(q)$ and $\mathrm{Sp}_{2 n}(q)$. The arguments are similar to those in Section 4 and will yield:

(1) the Hecke algebras associated to weakly cuspidal representations,

(2) the branching graph for the parabolic induction and restriction,

(3) derived equivalences between blocks.

The main difference is the lack of a description in terms of level 1 Fock spaces (given by Ennola duality, see $\$ 4$ ). In particular, the grading by the imaginary roots, which in Section 4 was deduced from the grading in the $\mathrm{GL}_{n}$ case, is constructed in types $\mathrm{B}, \mathrm{C}$ via some explicit combinatorics in terms of Lusztig's symbols. 
6.1. Definitions. Throughout this section and the following, $q$ is any prime power. Note however that we will assume that $q$ is odd starting from Section 6.3.

6.1.1. Odd-dimensional orthogonal groups. Let $J_{n}$ be as in $\$ 4.1$. It is the matrix of a non-degenerate quadratic form. The odd-dimensional orthogonal group $\mathbf{S O}_{2 n+1}=$ $\mathrm{SO}_{2 n+1}\left(\overline{\mathbb{F}}_{q}\right)$ is

$$
\mathrm{SO}_{2 n+1}\left(\overline{\mathbb{F}}_{q}\right)=\left\{\left.g \in \mathbf{S L}_{2 n+1}\right|^{t} g J_{2 n+1} g=J_{2 n+1}\right\} .
$$

It is a connected reductive group of type $B_{n}$. The standard Frobenius map $F=F_{q}$ on $\mathbf{G L}_{2 n+1}$ induces a Frobenius endomorphism on $\mathbf{S O}_{2 n+1}$. The finite orthogonal group $\mathrm{SO}_{2 n+1}(q)$ is given by

$$
\mathrm{SO}_{2 n+1}(q)=\left(\mathbf{S O}_{2 n+1}\right)^{F}
$$

The subgroup of diagonal matrices $\mathbf{T}$ (resp. upper-triangular matrices $\mathbf{B}$ ) in $\mathbf{S O}_{2 n+1}$ is a split maximal torus (resp. an $F$-stable Borel subgroup). Note that $\mathbf{T}=$ $\left\{\operatorname{diag}\left(t_{n}, \ldots, t_{1}, 1, t_{1}^{-1}, \ldots, t_{n}^{-1}\right)\right\}$. The Weyl group $W$ of $\left(\mathbf{S O}_{2 n+1}, \mathbf{T}\right)$ is a Weyl group of type $B_{n}$, and $F$ acts trivially on it. For numbering the simple reflections of $W$ we will take the following convention:

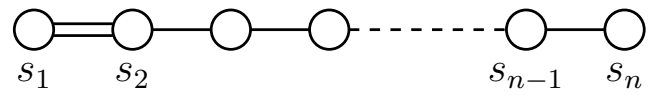

For $i \neq 1$, the action of $s_{i}$ on $\operatorname{diag}\left(t_{n}, \ldots, t_{1}, 1, t_{1}^{-1}, \ldots, t_{n}^{-1}\right)$ swaps $t_{i-1}$ and $t_{i}$, whereas $s_{1}$ swaps $t_{1}$ and $t_{1}^{-1}$. For $i \neq 1$, the simple reflection $s_{i}$ can be lifted to $N_{\mathbf{S O}_{2 n+1}}(\mathbf{T})$ as the permutation matrix $(n-i+1, n-i+2)(n+i, n+i+1)$ and $s_{1}$ as the signed permutation matrix

$$
s_{1}=-\left(\begin{array}{l|lll|l}
\operatorname{Id}_{n-1} & & & & \\
\hline & 0 & 0 & 1 & \\
& 0 & 1 & 0 & \\
& 1 & 0 & 0 & \\
\hline & & & & \operatorname{Id}_{n-1}
\end{array}\right) .
$$

6.1.2. Symplectic groups. Let $\widetilde{J}_{2 n}$ be the $2 n \times 2 n$ square matrix

$$
\widetilde{J}_{2 n}=\left(\begin{array}{cc}
0 & J_{n} \\
-J_{n} & 0
\end{array}\right) \text {. }
$$

It is the matrix of a symplectic form. The symplectic group $\mathbf{S p}_{2 n}=\operatorname{Sp}_{2 n}\left(\overline{\mathbb{F}}_{q}\right)$ is

$$
\mathbf{S p}_{2 n}=\left\{g \in \mathbf{G L}_{2 n} \mid{ }^{t} g \widetilde{J}_{2 n} g=\widetilde{J}_{2 n}\right\} .
$$


It is a connected reductive groups of type $C_{n}$. The standard Frobenius map $F=F_{q}$ on $\mathbf{G L}_{2 n}$ induces a Frobenius endomorphism on $\mathbf{S p}_{2 n}$. The finite symplectic group $\operatorname{Sp}_{2 n}(q)$ is given by

$$
\operatorname{Sp}_{2 n}(q)=\left(\mathbf{S p}_{2 n}\right)^{F} .
$$

The subgroup of diagonal matrices $\mathbf{T}$ (resp. upper-triangular matrices $\mathbf{B}$ ) in $\mathbf{S p}_{2 n}$ is a split maximal torus (resp. an $F$-stable Borel subgroup). Note that $\mathbf{T}=$ $\left\{\operatorname{diag}\left(t_{n}, \ldots, t_{1}, t_{1}^{-1}, \ldots, t_{n}^{-1}\right)\right\}$. The Weyl group $W$ of $\left(\mathbf{S p}_{2 n}, \mathbf{T}\right)$ is a Weyl group of type $B_{n}$, and $F$ acts trivially on it. For numbering the simple reflections of $W$ we will take the same convention as in (6.1).

As in the case of odd-dimensional orthogonal groups, the action of $s_{i}$ on the element $\operatorname{diag}\left(t_{n}, \ldots, t_{1}, t_{1}^{-1}, \ldots, t_{n}^{-1}\right)$ swaps $t_{i-1}$ and $t_{i}$ when $i \neq 1$, whereas $s_{1}$ swaps $t_{1}$ and $t_{1}^{-1}$. For $i \neq 1$, we will lift $s_{i}$ to $N_{\mathbf{S p}_{2 n}}(\mathbf{T})$ as the permutation matrix $(n-i+1, n-$ $i+2)(n+i-1, n+i)$ and $s_{1}$ as the signed permutation matrix

$$
s_{1}=\left(\begin{array}{c|cc|c}
\operatorname{Id}_{n-1} & & & \\
\hline & 0 & -1 & \\
& 1 & 0 & \\
\hline & & & \operatorname{Id}_{n-1}
\end{array}\right) .
$$

\subsection{The representation datum on $R G$-mod.}

Throughout this section we assume that $R$ is any commutative domain in which $q(q-1)$ is invertible.

Given a positive integer $n$, we will denote by $\mathbf{G}_{n}$ and $G_{n}$ either the odd-dimensional orthogonal groups $\mathbf{S O}_{2 n+1}$ and $\mathrm{SO}_{2 n+1}(q)$ or the symplectic groups $\mathbf{S p}_{2 n}$ and $\mathbf{S p}_{2 n}(q)$. In addition, we set $G_{0}=\{1\}$ by convention.

Given $r, m \in \mathbb{N}$ such that $n=r+m$, let $\mathbf{L}_{r, m}$ and $\mathbf{L}_{r, 1^{m}}$ be the standard Levi subgroups of $\mathbf{G}_{n}$ corresponding to the sets of simple reflexions $\left\{s_{k} \mid k \neq r+1\right\}$, $\left\{s_{k} \mid k \leq r\right\}$ respectively. The corresponding finite groups are $L_{r, m} \simeq G_{r} \times \mathrm{GL}_{m}(q)$ and $L_{r, 1^{m}} \simeq G_{r} \times \mathrm{GL}_{1}(q)^{m}$. We will abbreviate $\mathbf{L}_{r}=\mathbf{L}_{r, 1}$. It is isomorphic to $\mathbf{G}_{r} \times \mathbf{G L}_{1}$ via the map

$$
(g, \lambda) \longmapsto\left(\begin{array}{ccc}
\lambda & & \\
& g & \\
& & \lambda^{-1}
\end{array}\right) .
$$

Let $\mathbf{P}_{r} \subset \mathbf{G}_{r+1}$ be the corresponding parabolic subgroup and $P_{r}=\mathbf{P}_{r}^{F}$. Let $\mathbf{V}_{r}$ be the unipotent radical of $\mathbf{P}_{r}$ and $V_{r}=\mathbf{V}_{r}^{F}$. Let us consider the subgroup $U_{r} \subset G_{r+1}$ 
given by

$$
U_{r}=V_{r} \rtimes \mathbb{F}_{q}^{\times}
$$

It is represented by the same figure as in (4.1). For each $r<n$ we set $U_{n, r}=$ $U_{n-1} \rtimes \cdots \rtimes U_{r}$ and $e_{n, r}=e_{U_{n, r}}$. The embedding of $G_{r}$ into $L_{r}$ yields an embedding of $G_{r}$ into $G_{r+1}$, and by induction, of $G_{r}$ into $G_{n}$. We obtain functors

$$
\begin{gathered}
F_{n, r}=R G_{n} \cdot e_{n, r} \otimes_{R G_{r}} \bullet: R G_{r}-\bmod \rightarrow R G_{n}-\bmod , \\
E_{r, n}=e_{n, r} \cdot R G_{n} \otimes_{R G_{n}} \bullet: R G_{n}-\bmod \rightarrow R G_{r}-\bmod .
\end{gathered}
$$

An endomorphism of the functor $F_{n, r}$ can be represented by an $\left(R G_{n}, R G_{r}\right)$-bimodule endomorphism of $R G_{n} \cdot e_{n, r}$, or, equivalently, by an element of $e_{n, r} \cdot R G_{n} \cdot e_{n, r}$ centralizing $R G_{r}$. Thus, the elements

$$
X_{r+1, r}=q^{r} e_{r+1, r}\left(s_{r+1} s_{r} \cdots s_{1} \cdots s_{r} s_{r+1}\right) e_{r+1, r}, \quad T_{r+2, r}=q e_{r+2, r} s_{r+2} e_{r+2, r}
$$

define respectively natural transformations of the functors $F_{r+1, r}$ and $F_{r+2, r}$. Indeed, with our convention $s_{r+1} s_{r} \cdots s_{1} \cdots s_{r} s_{r+1}$ is one of the matrices

$$
-\left(\begin{array}{lll} 
& & 1 \\
& \operatorname{Id}_{G_{r}} & \\
1 & &
\end{array}\right) \quad \text { or } \quad\left(\begin{array}{lll} 
& & \\
& \operatorname{Id}_{G_{r}} & \\
1 & &
\end{array}\right)
$$

which centralize $G_{r}$. We set

$$
F=\bigoplus_{r \geqslant 0} F_{r+1, r}, \quad X=\bigoplus_{r \geqslant 0} X_{r+1, r}, \quad T=\bigoplus_{r \geqslant 0} T_{r+2, r} .
$$

Proposition 6.1. The endomorphisms $X \in \operatorname{End}(F)$ and $T \in \operatorname{End}\left(F^{2}\right)$ satisfy the following relations:

(a) $1_{F} T \circ T 1_{F} \circ 1_{F} T=T 1_{F} \circ 1_{F} T \circ T 1_{F}$,

(b) $\left(T+1_{F^{2}}\right) \circ\left(T-q 1_{F^{2}}\right)=0$,

(c) $T \circ\left(1_{F} X\right) \circ T=q X 1_{F}$.

Proof. Similar to the proof of [18, prop. 4.1].

\subsection{The categories of unipotent modules $\mathscr{U}_{K}$ and $\mathscr{U}_{\mathrm{k}}$.}

From now on, we fix an $\ell$-modular system $(K, \mathscr{O}, \mathrm{k})$ which we assume to be big enough so that every indecomposable unipotent representation of $G_{n}$ (over $K$ or k) is absolutely indecomposable. Such a modular system exists since unipotent representations of $G_{n}$ are defined over $\mathbb{Q}_{\ell}$, see [55, cor. 1.12]. In addition, since we will be dealing with representations in non-defining characteristic, we will always assume that $\ell \neq p$. We denote by $f$ (resp. $d$ ) the order of $q$ (resp. $q^{2}$ ) in $\mathrm{k}^{\times}$. If $f$ is odd, we 
say that the prime number $\ell$ is linear, otherwise we say that $\ell$ is unitary. In the first case we have $f=d$, whereas in the second one $f=2 d$.

From now on, we will always assume that both $p$ and $\ell$ are odd, and that $f>1$. In particular $q(q-1) \in \mathscr{O}^{\times}$and we can apply the previous constructions with $R$ being any ring among $(K, \mathscr{O}, \mathrm{k})$.

6.3.1. Parametrization by symbols. By [53], the unipotent characters of $G_{n}$ are parametrized by symbols. For our purpose it will be more convenient to work with a slightly different notion which we refer to as charged symbols.

A charged symbol $\Theta$ with charge $s=\left(s_{1}, s_{2}\right)$ is a pair of charged $\beta$-sets $\Theta=$ $\left(\beta_{s_{1}}\left(\mu^{1}\right), \beta_{s_{2}}\left(\mu^{2}\right)\right)$ for some bipartition $\mu=\left(\mu^{1}, \mu^{2}\right)$. We abbreviate $\Theta=\beta_{s}(\mu)$. If $\beta_{s_{1}}\left(\mu^{1}\right)=: X=\left\{x_{1}>x_{2}>\cdots\right\}$ and $\beta_{s_{2}}\left(\mu^{2}\right)=: Y=\left\{y_{1}>y_{2}>\cdots\right\}$ we write

$$
\Theta=(X, Y)=\left(\begin{array}{lll}
x_{1} & x_{2} & \cdots \\
y_{1} & y_{2} & \cdots
\end{array}\right) .
$$

The components $X$ and $Y$ are called the first and second row of the symbol. The defect of $\Theta$ is $D=s_{1}-s_{2}$ and its rank is $|\mu|+\left\lfloor D^{2} / 4\right\rfloor$.

A $d$-hook of $\Theta$ is a pair of integers $(x, x+d)$ which is either a $d$-hook of $X$ or a $d$-hook of $Y$. The charged symbol obtained by deleting $x+d$ from $X$ (resp. $Y$ ) and replacing it by $x$ is said to be gotten from $\Theta$ by removing the $d$-hook $(x, x+d)$. A $d$-cohook is a pair of integers $(x, x+d)$ such that $x+d \in X$ and $x \notin Y$, or $x+d \in Y$ and $x \notin X$. The charged symbol obtained by deleting $x+d$ from $X$ (resp. $Y$ ) and adding $x$ to $Y$ (resp. $X$ ) is said to be gotten from $\Theta$ by removing the $d$-cohook $(x, x+d)$. The $d$-core of $\Theta$ is obtained by removing recursively all $d$-hooks from $\Theta$. A similar definition using $d$-cohooks gives the $d$-cocore of $\Theta$.

We will denote by $\Theta^{\dagger}=(Y, X)$ the charged symbol of charge $\left(s_{2}, s_{1}\right)$ obtained by swapping the two $\beta$-sets. The defect of $\Theta^{\dagger}$ is $-D$ but the rank is the same. If one shifts simultaneously the charged $\beta$-sets $X$ and $Y$ by an integer $m$, one obtains a symbol $\Theta[m]$ of charge $\left(s_{1}+m, s_{2}+m\right)$. This operation does not change the defect nor the rank.

Symbols are orbits of charged symbols under the shift operator and the transformation $\Theta \mapsto \Theta^{\dagger}$. We write

$$
\{X, Y\}=\left\{\begin{array}{lll}
x_{1} & x_{2} & \cdots \\
y_{1} & y_{2} & \cdots
\end{array}\right\}
$$

for the symbol associated with $(X, Y)$. The rank of the symbol is the rank of any charged symbol in its class whereas its defect is $|D|$ where $D$ is the defect of any representative. Removing and adding $d$-hooks or $d$-cohooks are well-defined operations on symbols.

We denote by $\mathscr{S}$ the set of symbols and by $\mathscr{S}_{\text {odd }}$ the set of symbols of odd defects. 
6.3.2. The unipotent modules over $K$. Fix a positive integer $n$. The unipotent $K G_{n^{-}}$ modules were classified in [53, thm. 8.2]. They are parametrized in terms of symbols of odd defect and rank $n$. This parametrization follows from the determination of cuspidal unipotent $K G_{n}$-modules and their ramified Hecke algebras.

Proposition 6.2 (Lusztig [53]). Up to isomorphism, there is at most one cuspidal unipotent module in $K G_{r}$-mod, and there exists one if and only if $r=t(t+1)$ for some $t \geqslant 0$. It is denoted by $E_{t}$.

Since there do not exist any cuspidal unipotent character of $\mathrm{GL}_{n}(q)$ unless $n=1$, we deduce that any cuspidal pair of $G_{n}$ is conjugate to a pair of the form $\left(L_{r, 1^{m}}, E_{t}\right)$ with $n=r+m$ and $r=t(t+1)$ with $t \geqslant 0$. The general theory recalled in $\$ 3.4$ implies that the irreducible characters lying in the Harish-Chandra series above $E_{t}$ are in bijection with the irreducible representations of the ramified Hecke algebra $\mathscr{H}\left(K G_{n}, E_{t}\right)$. To describe explicitly the latter, we introduce, as in (4.3), the parameters

$$
Q_{t}=\left((-q)^{t},(-q)^{-1-t}\right) \text {. }
$$

Then $\mathscr{H}\left(K G_{n}, E_{t}\right)$ is isomorphic to $\mathbf{H}_{K, m}^{Q_{t} ; q}$ by [53, $\left.\S 5,8\right]$. There is a canonical choice for this isomorphism given by Theorem 6.5 below. Consequently, the Harish-Chandra theory yields a canonical bijection

$$
\operatorname{Irr}\left(K G_{n}, E_{t}\right) \stackrel{1: 1}{\longleftrightarrow} \operatorname{Irr}\left(\mathbf{H}_{K, m}^{Q_{t} ; q}\right)
$$

and hence a parametrization of $\operatorname{Irr}\left(K G_{n}, E_{t}\right)$. More precisely, given $\mu=\left(\mu^{1}, \mu^{2}\right)$ a bipartition of $m$ and $t \geqslant 0$ we can associate the symbol

$$
\Theta_{t}(\mu)=\left\{\beta_{t}\left(\mu^{1}\right), \beta_{-t-1}\left(\mu^{2}\right)\right\} .
$$

So, the defect and the rank of $\Theta_{t}(\mu)$ are

$$
D\left(\Theta_{t}(\mu)\right)=2 t+1, \quad \operatorname{rk}\left(\Theta_{t}(\mu)\right)=m+t(t+1) .
$$

Then we define $E_{\Theta_{t}(\mu)}$ to be the unipotent $K G_{r}$-module corresponding to $S(\mu)_{K}^{Q_{t} ; q}$ via the bijection (6.4). This yields a parametrization of the irreducible unipotent $K G_{n}$-modules as

$$
\left\{E_{\Theta_{t}(\mu)} \mid t \in \mathbb{N}, \mu \in \mathscr{P}_{m}^{2}, m+t(t+1)=n\right\} .
$$

We will abbreviate $\Theta_{t}=\Theta_{t}(\emptyset)$. Note that $E_{t}=E_{\Theta_{t}}$.

Proposition 6.3 (Lusztig [53]). The irreducible unipotent characters are parametrized by symbols of odd defect. If $n=r+m$ and $r=t(t+1)$ with $t \geqslant 0$, then the unipotent module $E_{\Theta}$ lies in the Harish-Chandra series $\operatorname{Irr}\left(K G_{n}, E_{t}\right)$ if and only if $\Theta$ has defect $2 t+1$ and rank $n$. 
It is important to observe that this parametrization is exactly the one described in [53, thm. 8.2], which was subsequently used for the determination of blocks in [23]. Indeed, the labelling of unipotent characters by bipartitions or symbols in [53] (see also $[9, \S 13.8])$ corresponds to the presentation of the Hecke algebra $\mathscr{H}\left(K G_{n}, E_{t}\right)$ in terms of the set of generators $\left(T_{0}, T_{1}, \ldots, T_{m-1}\right)$ instead of $\left(X_{1}, T_{1}, \ldots, T_{m-1}\right)$ with $T_{0}=(-1)^{t} q^{t+1} X_{1}$ so that $\left(T_{0}-q^{2 t+1}\right)\left(T_{0}+1\right)=0$. Now the isomorphism $\mathbf{H}_{K, m}^{Q_{t} ; q} \simeq$ $\mathbf{H}_{K, m}^{\left(q^{2 t+1},-1\right) ; q}$ induces a bijection on irreducible objects which is just $S(\mu)^{Q_{t}} \longmapsto$ $S(\mu)^{\left(q^{2 t+1},-1\right)}$.

Remark 6.4. Note that $Q_{t}^{\dagger}=Q_{-1-t}, \Theta_{t}(\mu)=\Theta_{-1-t}\left(\mu^{\dagger}\right)$ and $t(t+1)$ is invariant under the map $t \longmapsto-t-1$. We will work with symbols $\Theta_{t}(\mu)$ such that $t \geqslant 0$. We might also have worked with $t<0$ using the symmetries above.

Recall that $G_{0}=\{1\}$. We call the category of unipotent $K G$-modules the category

$$
\mathscr{U}_{K}=\bigoplus_{n \in \mathbb{N}} K G_{n}-\operatorname{umod}
$$

This category is abelian semisimple. From the previous discussion we have

$$
\operatorname{Irr}\left(\mathscr{U}_{K}\right)=\left\{E_{\Theta} \mid \Theta \in \mathscr{S}_{\text {odd }}\right\}
$$

where by convention $\operatorname{Irr}\left(K G_{0}\right)=\left\{E_{0}\right\}$.

6.3.3. The unipotent modules over $\mathrm{k}$. Using the $\ell$-modular system we have decomposition maps $d_{\mathscr{O} G_{n}}$ which by Proposition 3.4 and since $\ell$ is odd, restrict to linear isomorphisms

$$
d_{\mathscr{O} G_{n}}:\left[K G_{n}-\mathrm{umod}\right] \stackrel{\sim}{\rightarrow}\left[\mathrm{k} G_{n} \text {-umod }\right] .
$$

We call the category of unipotent $\mathrm{k} G$-modules the category

$$
\mathscr{U}_{\mathrm{k}}=\bigoplus_{n \in \mathbb{N}} \mathrm{k} G_{n}-\text { umod }
$$

This is an abelian category which is not semisimple. As above, the decomposition map yields a $\mathbb{Z}$-linear isomorphism $d_{\mathscr{U}}:\left[\mathscr{U}_{K}\right] \stackrel{\sim}{\rightarrow}\left[\mathscr{U}_{\mathrm{k}}\right]$. It is conjectured that this map is unitriangular on the basis of irreducible modules, yielding a parametrization of unipotent simple $\mathrm{k} G$-modules (see Conjecture 6.21 below).

6.3.4. The unipotent blocks. Recall that $d$ is the order of $q^{2}$ modulo $\ell$. The partition of the unipotent $K G_{n}$-modules into $\ell$-blocks was determined in [23, (10B), (11E)]. If $\ell$ is a linear prime (resp. a unitary prime), i.e., if $f$ is odd (resp. even), then two unipotent characters of $K G_{n}$ belong to the same $\ell$-block if and only if their symbols have the same $d$-core (resp. $d$-cocore). 
In addition, Fong-Srinivasan described the structure of the unipotent blocks of $G_{n}$ with cyclic defect groups. First, the unipotent $\ell$-block containing $E_{\Theta}$ has a cyclic defect if and only if the symbol $\Theta$ has a unique $d$-hook if $\ell$ is linear or a unique $d$-cohook if $\ell$ is unitary. In this case, let $\{X, Y\}$ be the $d$-core of $\Theta$ if $\ell$ is linear, and the $d$-cocore of $\Theta$ if $\ell$ is unitary. By [24, (5A), (6A)], the Brauer tree of the $\ell$-block containing $E_{\Theta}$ is

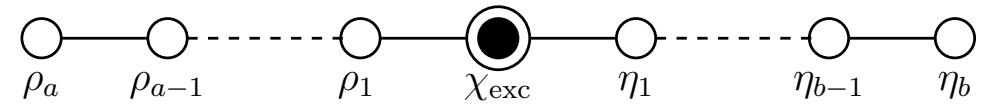

where

(a) if $\ell$ is linear, then $f=d=a=b$ and $\rho_{k}$ (resp. $\eta_{k}$ ) is obtained by adding a $d$-hook to $X$ (resp. $Y$ ) for each $k=1, \ldots, d$.

(b) if $\ell$ is unitary, then $f=2 d, \rho_{1}, \ldots, \rho_{a}$ are the unipotent characters corresponding to the symbols obtained by adding a $d$-cohook which increases $|X|$ and $\eta_{1}, \ldots, \eta_{b}$ are the unipotent characters corresponding to the symbols obtained by adding a $d$-cohook which increases $|Y|$. In addition, $a=d+D$ and $b=d-D$ where $D$ is the defect of the symbol.

\subsection{The $\mathfrak{g}_{\infty}$-representation on $\mathscr{U}_{K}$.}

We show in this section that the representation datum on $K G$-mod yields a categorical action of $\mathfrak{g}_{\infty}$ on $\mathscr{U}_{K}$.

6.4.1. The ramified Hecke algebra. Let $r=t(t+1)$ and $n=r+m$ with $t, m \geqslant 0$. Recall that the inflation from $G_{r}$ to $L_{r, 1^{m}}$ yields an equivalence between $K G_{r}$-umod and $K L_{r, 1^{m}}$-umod which intertwines the functor $F_{n, r}$ with the parabolic induction $R_{L_{r, 1}}^{G_{n}}$. In particular, we have a canonical isomorphism

$$
\mathscr{H}\left(K G_{n}, E_{t}\right):=\operatorname{End}_{K G_{n}}\left(F^{m}\left(E_{t}\right)\right)^{\text {op }} \stackrel{\sim}{\rightarrow} \operatorname{End}_{K G_{n}}\left(R_{L_{r, 1}}^{G_{n}}\left(E_{t}\right)\right)^{\text {op }} .
$$

This algebra is isomorphic to the Hecke algebra of type $B_{m}$ with parameters $q$ and $Q_{t}$, giving a parametrisation of the constituents of (the head of) $F^{m}\left(E_{t}\right)$ in terms of bipartitions. More precisely, recall from $\$ 1.3$ that to the categorical datum $(E, F, X, T)$ is attached a map $\phi_{F^{m}}: \mathbf{H}_{K, m}^{q} \rightarrow \operatorname{End}\left(F^{m}\right)$. The evaluation of this map at the module $E_{t}$ yields a $K$-algebra homomorphism

$$
\phi_{K, m}: \mathbf{H}_{K, m}^{q} \rightarrow \mathscr{H}\left(K G_{n}, E_{t}\right), \quad X_{k} \mapsto X_{k}\left(E_{t}\right), \quad T_{l} \mapsto T_{l}\left(E_{t}\right) .
$$

We show that it induces the aforementioned isomorphism.

Theorem 6.5. Let $t, m \geqslant 0$ and $n=t(t+1)+m$. Then the map $\phi_{K, m}$ factors through a $K$-algebra isomorphism $\mathbf{H}_{K, m}^{Q_{t} ; q} \stackrel{\sim}{\longrightarrow} \mathscr{H}\left(K G_{n}, E_{t}\right)$. 
Proof. The proof is similar to the proof of Theorem 4.8, see [18, thm. 4.12]. Write $Q_{t}=\left(Q_{1}, Q_{2}\right)$ and $X=X\left(E_{t}\right)$. We must check that the operator $X$ on $F\left(E_{t}\right)$ satisfies the relation

$$
\left(X-(-q)^{-1-t}\right)\left(X-(-q)^{t}\right)=0 .
$$

Then the invertibility of the morphism $\mathbf{H}_{K, m}^{Q_{t} ; q} \rightarrow \mathscr{H}\left(K G_{n}, E_{t}\right)$ follows from [42]. In fact, it is shown there that $X$ satifies the relation

$$
\left(X-\epsilon_{t}(-q)^{-1-t}\right)\left(X-\epsilon_{t}(-q)^{t}\right)=0
$$

for some $\epsilon_{t}= \pm 1$. Therefore we must show that $\epsilon_{t}=1$ for all $t \geq 0$, which we will do by induction on $t$. First observe that the eigenvalues of $X_{1,0}$ on $R_{G_{0}}^{G_{1}}(K)$ are $1,(-q)^{-1}$ and thus are powers of $-q$. Now fix $t \geq 1$ and assume that for all $t>s \geqslant 0$ the eigenvalues of $X\left(E_{s}\right)$ on $F\left(E_{s}\right)$ are powers of $-q$. We will show that the eigenvalues of $X\left(E_{t}\right)$ are also powers of $-q$ using the modular representation theory of $G_{n}$.

Recall that $K$ is chosen with respect to an $\ell$-modular system $(K, \mathscr{O}, \mathrm{k})$. Since the parametrization of unipotent characters does not depend on $\ell$ and $(E, F, X, T)$ are defined over $\mathbb{Z}[1 / q(q-1)]$, we can first choose a specific prime number $\ell$ and prove that the eigenvalues of $X\left(E_{t}\right)$ are powers of $-q$ modulo $\ell$. We choose $\ell$ to be odd and such that the order of $q$ in $\mathrm{k}^{\times}$is $f:=4 t$. Thus the order of $q^{2}$ is $d=2 t$. Note that $\ell$ is a unitary prime.

Set $r=t(t+1)$ and $n=r+1$. The cuspidal representation $E_{t}$ is attached to the symbol

$$
\Theta_{t}=\left\{\begin{array}{lllllll}
t & t-1 & t-2 & \cdots & -t & -t-1 & \cdots \\
& & & & & -t-1 & \cdots
\end{array}\right\}
$$

Since only one $d$-cohook can be removed from $\Theta_{t}$, the $\ell$-block of $E_{t}$ has cyclic defect groups. Moreover, the $d$-cocore of $\Theta_{t}$ equals $\Theta_{t-1}$. Consequently, the Brauer tree of the $\ell$-block of $E_{t}$ is

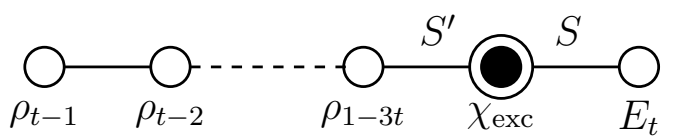

where a symbol $\Xi_{k}$ of $\rho_{k}$ is obtained by adding the $d$-cohook $(k, k+d)$ to $\Theta_{t-1}$ for $k \in\{1-3 t, \ldots, t-1\}$. Explicitly, we have

$$
\Xi_{k}=\left\{\begin{array}{cccccc}
t-1 & t-2 & \ldots & \widehat{k} & \ldots & \ldots \\
& & k+2 t & -t & -t-1 & \cdots
\end{array}\right\} .
$$

Here the notation $\widehat{k}$ means that the integer $k$ has been removed. This symbol has defect $|2 t-3|=|2(t-2)+1|$. Therefore by Proposition 6.3, the unipotent characters $\rho_{k}$ all lie in the Harish-Chandra series above $E_{t-2}$ (recall the convention $E_{-1}:=E_{0}$ ). 
Furthermore, the bipartition $\mu_{k}$ such that $\Theta_{t-2}\left(\mu_{k}\right)=\Xi_{k}$ is $\left(\left(1^{t-1-k}\right),(k+3 t-1)\right)$, except when $t=1$ in which case $\mu_{k}=\left((k+2),\left(1^{-k}\right)\right)$.

The $K G_{r}$-module $E_{t}$ is cuspidal, hence weakly cuspidal. It follows that all the composition factors of any $\ell$-reduction of $E_{t}$ are weakly cuspidal as well. In particular

$$
E(S)=0 .
$$

For each $k$ we can compute the character $E\left(\rho_{k}\right)$ obtained by removing a 1-hook from $\Xi_{k}$. Two cases arise: if $k=1-3 t$ or $k=t-1$, then $E\left(\rho_{k}\right)$ is irreducible, and the corresponding symbols are equal respectively to

and

$$
\begin{aligned}
& \left\{\begin{array}{cccccc}
t-1 & t-2 & \ldots & \widehat{-3 t+2} & \ldots & \ldots \\
& & -t+1 & -t & -t-1 & \ldots
\end{array}\right\}, \\
& \left\{\begin{array}{ccccc}
t-2 & \ldots & \ldots & \ldots & \ldots \\
& 3 t-2 & -t & -t-1 & \ldots
\end{array}\right\} ;
\end{aligned}
$$

otherwise $E\left(\rho_{k}\right)$ has two constituents whose symbols are

and

$$
\begin{aligned}
& \left\{\begin{array}{ccccc}
t-1 & t-2 & \ldots & \widehat{k+1} & \ldots \\
& k+2 t & -t & -t-1 & \ldots
\end{array}\right\}, \\
& \left\{\begin{array}{cccccc}
t-1 & t-2 & \cdots & \widehat{k} & \cdots \\
& k+2 t-1 & -t & -t-1 & \cdots
\end{array}\right\} .
\end{aligned}
$$

We deduce that $\sum(-1)^{k}\left[E\left(\rho_{k}\right)\right]=0$ in $\left[K G_{r-1}\right.$-mod $]$. Since $\left[S^{\prime}\right]=\sum(-1)^{k} d_{\mathscr{U}}\left(\left[\rho_{k}\right]\right)$ in $\left[\mathrm{k} G_{r-1}-\mathrm{mod}\right]$, this implies that

$$
E\left(S^{\prime}\right)=0
$$

Therefore, the two composition factors $S, S^{\prime}$ of the $\ell$-reduction of the exceptional characters are weakly cuspidal, which forces the exceptional characters to be weakly cuspidal as well.

Given a symbol $\Theta$, the module $F\left(E_{\Theta}\right)$ is the sum of the unipotent characters associated to the symbols obtained from $\Theta$ by adding a 1-hook. Thus, we have $F\left(E_{t}\right)=E_{\Xi} \oplus E_{\Xi^{\prime}}$, where

$$
\begin{aligned}
& \Xi=\left\{\begin{array}{lllllll}
t & t-1 & \cdots & -t & -t-1 & -t-2 & \cdots \\
& & -t & -t-2 & \cdots
\end{array}\right\}, \\
& \Xi^{\prime}=\left\{\begin{array}{llllll}
t+1 & t-1 & t-2 & \cdots & -t-1 & \cdots \\
& & & & -t-1 & \cdots
\end{array}\right\} .
\end{aligned}
$$

Let $B, B^{\prime}$ be the $\ell$-blocks of $E_{\Xi}$ and $E_{\Xi^{\prime}}$ respectively and $b, b^{\prime}$ be the corresponding idempotents in $K G_{n}$. Since the $d$-cocores of $\Xi$ and $\Xi^{\prime}$ are different, the idempotents $b$ and $b^{\prime}$ are orthogonal. Moreover $X\left(E_{t}\right)$ has the eigenvalues $\epsilon_{t}(-q)^{-1-t}$ and $\epsilon_{t}(-q)^{t}$, 
on $b F\left(E_{t}\right)=E_{\Xi}$ and $b^{\prime} F\left(E_{t}\right)=E_{\Xi^{\prime}}$ respectively. Note that $\epsilon_{t}(-q)^{-1-t}$ and $\epsilon_{t}(-q)^{t}$ are not congruent modulo $\ell$ since $q^{2 t+1} \equiv-q$.

Let $\chi$ be an exceptional character. Recall that $S$ is isomorphic to any $\ell$-reduction of $E_{t}$. Since it is a composition factor of the $\ell$-reduction of $\chi$, we deduce that both $b F(\chi)$ and $b^{\prime} F(\chi)$ are non-zero. Since $\chi$ is weakly cuspidal, by the Mackey formula $F(\chi)$ has at most two irreducible constituents and $X(\chi)$ at most two eigenvalues whose product equals $-q^{-1}$. We deduce that $F(\chi)=b F(\chi) \oplus b^{\prime} F(\chi)$. In addition, the eigenvalues of $X(\chi)$ must be congruent to the eigenvalues of $X(S)$ on $F(S)$, which are equal to $\epsilon_{t}(-q)^{-1-t}$ and $\epsilon_{t}(-q)^{t}$. Now, $S^{\prime}$ is a composition factor of the $\ell$-reduction of $\chi$ so one of $b F\left(S^{\prime}\right)$ or $b^{\prime} F\left(S^{\prime}\right)$ must be non-zero, and therefore $X\left(S^{\prime}\right)$ must have an eigenvalue congruent to $\epsilon_{t}(-q)^{-1-t}$ or $\epsilon_{t}(-q)^{t}$. To conclude that $\epsilon_{t}=1$, we must compute the possible eigenvalues of $X\left(\rho_{1-3 t}\right)$ on $b F\left(\rho_{1-3 t}\right)$ and $b^{\prime} F\left(\rho_{1-3 t}\right)$ and use that $S^{\prime}$ is a composition factor of the $\ell$-reduction of $\rho_{1-3 t}$.

We abbreviate $\rho=\rho_{1-3 t}$. The Harish-Chandra induction of $\rho$ is $F(\rho)=E_{\Theta} \oplus$ $E_{\Theta^{\prime}} \oplus E_{\Theta^{\prime \prime}}$ where

$$
\begin{aligned}
\Theta & =\left\{\begin{array}{llllll}
t & t-2 & t-3 & \ldots & \widehat{1-3 t} & \ldots \\
& & -t+1 & -t & \cdots
\end{array}\right\}, \\
\Theta^{\prime} & =\left\{\begin{array}{llllll}
t-1 & t-2 & \ldots & \widehat{-3 t} & \cdots & \cdots \\
& & -t+1 & -t & -t-1 & \cdots
\end{array}\right\}, \\
\Theta^{\prime \prime} & =\left\{\begin{array}{llllll}
t-1 & t-2 & \ldots & \widehat{1-3 t} & \cdots & \ldots \\
& & -t+2 & -t & -t-1 & \cdots
\end{array}\right\} .
\end{aligned}
$$

We first observe that the modules $E_{\Theta}, E_{\Theta^{\prime}}$ belong to the $\ell$-blocks $B, B^{\prime}$ and that $E_{\Theta^{\prime \prime}}$ is projective. Indeed, the $d$-cocores of the symbols $\Theta, \Theta^{\prime}$ and $\Theta^{\prime \prime}$ are different and equal respectively to

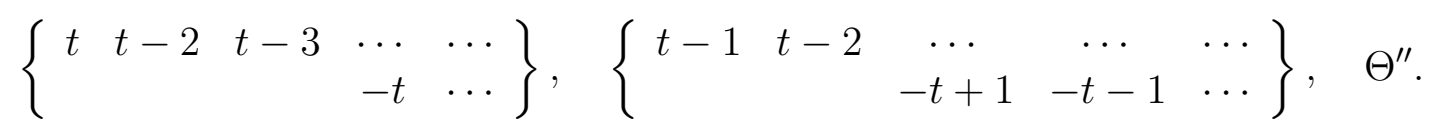

Since $\rho$ belong to the Harish-Chandra series above $E_{t-2}$, we can compute the eigenvalues of $X(\rho)$ using the eigenvalues of $X\left(E_{t-2}\right)$. Let $n^{\prime}=r+m-4 t+2$ for some integer $m \geqslant 0$. If $t \geq 2$ we can use the induction hypothesis and the map $\phi_{K, m}$ yields a $K$-algebra isomorphism

$$
\mathbf{H}_{K, m}^{Q_{t-2}, q} \stackrel{\sim}{\rightarrow} \mathscr{H}\left(K G_{n^{\prime}}, E_{t-2}\right) .
$$

By the discussion after (6.4), the corresponding bijection

$$
\operatorname{Irr}\left(K G_{n^{\prime}}, E_{t-2}\right) \stackrel{1: 1}{\longleftrightarrow} \operatorname{Irr}\left(\mathbf{H}_{K, m}^{Q_{t-2}, q}\right)
$$

takes the module $E_{\Theta_{t-2}(\mu)}$ to $S(\mu)_{K}^{Q_{t-2}, q}$ for each 2-partition $\mu$ of $m$. Under this parametrization, the character $\rho$ of $K G_{r}$ and the characters $E_{\Theta}, E_{\Theta^{\prime}}$ and $E_{\Theta^{\prime \prime}}$ of 
$K G_{n}$ are mapped to the modules

$$
S(\lambda)_{K}^{Q_{t-2}, q}, \quad S(\mu)_{K}^{Q_{t-2}, q}, \quad S\left(\mu^{\prime}\right)_{K}^{Q_{t-2}, q}, \quad S\left(\mu^{\prime \prime}\right)_{K}^{Q_{t-2}, q}
$$

labelled by the following 2-partitions

$$
\lambda=\left(\left(1^{4 t-2}\right), \emptyset\right), \quad \mu=\left(\left(21^{4 t-3}\right), \emptyset\right), \quad \mu^{\prime}=\left(\left(1^{4 t-1}\right), \emptyset\right), \quad \mu^{\prime \prime}=\left(\left(1^{4 t-2}\right),(1)\right) .
$$

The $\left(Q_{t-2}, q\right)$-shifted residue of the boxes $Y(\mu) \backslash Y(\lambda)$ and $Y\left(\mu^{\prime}\right) \backslash Y(\lambda)$ are $(-1)^{t} q^{t-1}$ and $(-1)^{t} q^{-3 t}$ respectively. They are congruent to $(-q)^{-1-t}$ and $(-q)^{t}$ modulo $\ell$, because $q^{2 t}$ is congruent to -1 modulo $\ell$. We deduce that the eigenvalues of the operator $X\left(\rho_{1-3 t}\right)$ on $E_{\Theta}, E_{\Theta^{\prime}}$ are congruent to $(-q)^{-1-t}$ and $(-q)^{t}$ modulo $\ell$. By the above argument, at least one of these must be congruent to $\epsilon_{t}(-q)^{-1-t}$ or $\epsilon_{t}(-q)^{t}$, which forces $\epsilon_{t}=1$.

Finally, if $t<2$ (in which case $t=1$ ) we use the fact that $E_{t-2}=E_{1-t}$ and $Q_{t-2}=Q_{1-t}^{\dagger}$. In particular, the bijection $\operatorname{Irr}\left(K G_{n^{\prime}}, E_{t-2}\right) \rightarrow \operatorname{Irr}\left(\mathbf{H}_{K, m}^{Q_{1-t}, q}\right)$ takes the module $E_{\Theta_{t-2}(\mu)}$ to $S\left(\mu^{\dagger}\right)_{K}^{\left(Q_{1-t}, q\right)}$ for any bipartition $\mu$ of $m$. With $t=1$, we deduce that the unipotent characters $\rho, E_{\Theta}, E_{\Theta^{\prime}}$ and $E_{\Theta^{\prime \prime}}$ correspond to Specht modules of $\mathbf{H}_{K, m}^{Q_{0}, q}$ labelled by the 2-partitions $\lambda=\left(\emptyset,\left(1^{2}\right)\right), \mu=(\emptyset,(21)), \mu^{\prime}=\left(\emptyset,\left(1^{3}\right)\right)$ and $\mu^{\prime \prime}=\left((1),\left(1^{2}\right)\right)$. With $Q_{0}=\left(1,-q^{-1}\right)$, the $\left(Q_{0}, q\right)$-shifted residues of the boxes $Y(\mu) \backslash Y(\lambda)$ and $Y\left(\mu^{\prime}\right) \backslash Y(\lambda)$ are -1 and $-q^{-3}$ respectively, which are congruent to $q^{-2}$ and $-q$ since $q^{2}$ is congruent to -1 modulo $\ell$. Therefore $\epsilon_{1}=1$.

6.4.2. The $\mathfrak{g}_{\infty}$-representation on $\mathscr{U}_{K}$. By Proposition 6.1, the Harish-Chandra induction and restriction yield a representation datum $(E, F, X, T)$ on the category

$$
\mathscr{U}_{K}=\bigoplus_{n \in \mathbb{N}} K G_{n} \text {-umod }
$$

For any $t, m, n \in \mathbb{N}$, let $\left(K G_{n}, E_{t}\right)$-mod be the Serre subcategory of $\mathscr{U}_{K}$ generated by the modules $F^{m}\left(E_{t}\right)$ with $n=r+m$ and $r=t(t+1)$. Write

$$
\mathscr{U}_{K, t}:=\bigoplus_{n \geqslant 0}\left(K G_{n}, E_{t}\right)-\bmod
$$

We have $\operatorname{Irr}\left(\left(K G_{n}, E_{t}\right)-\bmod \right)=\operatorname{Irr}\left(K G_{n}, E_{t}\right)$ and $\mathscr{U}_{K}=\bigoplus_{t \geqslant 0} \mathscr{U}_{K, t}$.

Definition 6.6. Let $I_{\infty}$ denote the subset $q^{\mathbb{Z}} \sqcup\left(-q^{\mathbb{Z}}\right)$ of $K^{\times}$. We define $\mathfrak{g}_{\infty}$ to be the (derived) Kac-Moody algebra associated to the quiver $I_{\infty}(q)$.

To avoid cumbersome notation, we will write $I_{\infty}=I_{\infty}(q)$. We denote by $\left\{\Lambda_{i}\right\}$, $\left\{\alpha_{i}\right\}$ and $\left\{\alpha_{i}^{\vee}\right\}$ the fundamental weights, simple roots and simple coroots of $\mathfrak{g}_{\infty}$. Then $\mathrm{X}_{\infty}=\mathrm{P}_{\infty}=\bigoplus \mathbb{Z} \Lambda_{i}$. There is a Lie algebra isomorphism $\left(\mathfrak{s l}_{\mathbb{Z}}\right)^{\oplus 2} \stackrel{\sim}{\rightarrow} \mathfrak{g}_{\infty}$ such 
that $\left(\alpha_{k}^{\vee}, 0\right) \mapsto \alpha_{q^{k}}^{\vee}$ and $\left(0, \alpha_{k}^{\vee}\right) \mapsto \alpha_{-q^{k}}^{\vee}$. Since the pair $Q_{t}$ belongs to $\left(I_{\infty}\right)^{2}$, the $\mathfrak{g}_{\infty}$-module $\mathbf{F}\left(Q_{t}\right)_{\infty}$ is well-defined.

Theorem 6.7. Let $t \geqslant 0$ and $Q_{t}$ be as in (6.3).

(a) The Harish-Chandra induction and restriction functors yield a representation of $\mathfrak{g}_{\infty}$ on $\mathscr{U}_{K, t}$ which is isomorphic to $\mathscr{L}\left(\Lambda_{Q_{t}}\right)_{\infty}$.

(b) The map $\left|\mu, Q_{t}\right\rangle_{\infty} \mapsto\left[E_{\Theta_{t}(\mu)}\right]$ gives a $\mathfrak{g}_{\infty}$-module isomorphism $\mathbf{F}\left(Q_{t}\right)_{\infty} \stackrel{\sim}{\rightarrow}\left[\mathscr{U}_{K, t}\right]$.

Proof. The proof is similar to the proof of Theorem 4.11 in [18, thm. 4.15]. It is a consequence of Theorem 6.5 and the discussion after (6.4).

\subsection{The $\mathfrak{g}_{2 d}$-representation on $\mathscr{U}_{\mathrm{k}}$.}

We now turn to the case of unipotent representations in positive characteristic. We first deduce from the previous construction a categorical action of the derived Kac-Moody algebra $\mathfrak{g}_{2 d}^{\prime}$ on $\mathscr{U}_{\mathrm{k}}$. We then show how to extend this action depending on the parity of $f$. This reflects the difference between the case of linear primes $(f$ odd) and unitary primes ( $f$ even).

6.5.1. The $\mathfrak{g}_{2 d}^{\prime}$-representation on $\mathscr{U}_{\mathrm{k}}$. Recall that $(K, \mathscr{O}, \mathrm{k})$ is an $\ell$-modular system with $K \supset \mathbb{Q}_{\ell}$ and $\mathrm{k} \supset \overline{\mathbb{F}}_{\ell}$. We still work under the assumption that $\ell \nmid q$ and $\ell$ and $q$ are odd. Recall that $d$ (resp. $f$ ) is the order of $q^{2}$ (resp. $q$ ) modulo $\ell$.

Definition 6.8. Let $I_{2 d}$ be the quiver obtained from $I_{\infty}$ by specialization $\mathscr{O} \rightarrow \mathrm{k}$. We define $\mathfrak{g}_{2 d}^{\prime}$ to be the derived Kac-Moody algebra associated to the quiver $I_{2 d}$.

If $f$ is odd then $f=d$ and -1 cannot be expressed as a power of $q$ in $\mathrm{k}$. In that case the quiver $I_{2 d}$ decomposes as $I_{2 d}=I_{d, 1} \sqcup I_{d, 2}$ with $I_{d, 1}=q^{\mathbb{Z}}$ and $I_{d, 2}=-q^{\mathbb{Z}}$ being both cyclic quivers of size $d$. This yields a Lie algebra isomorphism

$$
\left(\widetilde{\mathfrak{s l}}_{d}\right)^{\oplus 2} \simeq \mathfrak{g}_{d, 1}^{\prime} \oplus \mathfrak{g}_{d, 2}^{\prime}=\mathfrak{g}_{2 d}^{\prime}
$$

such that $\left(\alpha_{k}^{\vee}, 0\right) \mapsto \alpha_{q^{k}}^{\vee}$ and $\left(0, \alpha_{k}^{\vee}\right) \mapsto \alpha_{-q^{k}}^{\vee}$.

If $f$ is even, then $f=2 d$ and $q^{d}=-1$. Hence $I_{2 d}=q^{\mathbb{Z}}$ is a cyclic quiver of size $2 d$ and we have an isomorphism $\widetilde{\mathfrak{s l}}_{f} \simeq \mathfrak{g}_{2 d}^{\prime}$ such that $\alpha_{k}^{\vee} \mapsto \alpha_{q^{k}}^{\vee}$.

The specialization from $\mathscr{O} \subset K$ to k yields a morphism of quivers sp $: I_{\infty} \rightarrow I_{2 d}$ and a morphism of abelian groups $\mathrm{P}_{\infty} \rightarrow \mathrm{P}_{2 d}$ such that $\Lambda_{i} \mapsto \Lambda_{\mathrm{sp}(i)}$. The infinite sums $E_{i}=\bigoplus_{\mathrm{sp}(j)=i} E_{j}$ and $F_{i}=\bigoplus_{\mathrm{sp}(j)=i} F_{j}$ give well-defined operators on $\mathbf{F}\left(Q_{t}\right)_{\infty}$. This yields a representation of $\mathfrak{g}_{2 d}^{\prime}$ on $\mathbf{F}\left(Q_{t}\right)_{\infty}$ such that the linear map

$$
\text { sp : } \operatorname{Res}_{\mathfrak{g}_{2 d}^{\prime}}^{\mathfrak{g}_{\infty}} \mathbf{F}\left(Q_{t}\right)_{\infty} \rightarrow \mathbf{F}\left(Q_{t}\right)_{2 d}
$$

given by $\left|\mu, Q_{t}\right\rangle_{\infty} \mapsto\left|\mu, Q_{t}\right\rangle_{2 d}$ is a $\mathfrak{g}_{2 d^{\prime}}^{\prime}$ equivariant isomorphism. 
Under the map $d_{\mathscr{U}}:\left[\mathscr{U}_{K}\right] \rightarrow\left[\mathscr{U}_{\mathrm{k}}\right]$ and the isomorphism $\bigoplus_{t \in \mathbb{N}} \mathbf{F}\left(Q_{t}\right)_{\infty} \stackrel{\sim}{\rightarrow}\left[\mathscr{U}_{K}\right]$ in Theorem 6.7, the map sp endows $\left[\mathscr{U}_{\mathrm{k}}\right]$ with a representation of $\mathfrak{g}_{2 d}^{\prime}$ which is compatible with the representation associated with the representation datum. More precisely, the following hold.

Proposition 6.9. For each $i \in I_{2 d}$, let $\mathrm{k} E_{i}$ and $\mathrm{k} F_{i}$ be the generalized $i$-eigenspace of $X$ on $\mathrm{k} E$ and $\mathrm{k} F$. Then

(a) $\left[\mathrm{k} E_{i}\right],\left[\mathrm{k} F_{i}\right]$ endow $\left[\mathscr{U}_{\mathrm{k}}\right]$ with a structure of $\mathfrak{g}_{2 d^{\prime}}^{\prime}$-module,

(b) $d_{\mathscr{U}}$ yields a $\mathfrak{g}_{2 d}^{\prime}$-module isomorphism $\operatorname{Res}_{\mathfrak{g}_{2 d}^{\prime}}^{\mathfrak{g}_{\infty}}\left[\mathscr{U}_{K}\right] \stackrel{\sim}{\rightarrow}\left[\mathscr{U}_{\mathrm{k}}\right]$,

(c) the map $\left|\mu, Q_{t}\right\rangle_{2 d} \mapsto d_{\mathscr{U}}\left(\left[E_{\Theta_{t}(\mu)}\right]\right)$ yields a $\mathfrak{g}_{2 d}^{\prime}$-module isomorphism

$$
\bigoplus_{t \in \mathbb{N}} \mathbf{F}\left(Q_{t}\right)_{2 d} \stackrel{\sim}{\rightarrow}\left[\mathscr{U}_{\mathrm{k}}\right]
$$

Proof. The proposition is a direct application of Theorem 6.7, once we have proved that the decomposition map $d_{\mathscr{U}}$ is a vector space isomorphism. This is known by Theorem 3.4 since $\ell$ is odd.

In order to prove that this, and the representation datum $(E, F, X, T)$ on $\mathscr{U}_{\mathrm{k}}$ introduced in $\S 6.2$, endow $\mathscr{U}_{\mathrm{k}}$ with a $\mathfrak{g}_{2 d}^{\prime}$-representation, it remains to see that weight spaces are sums of blocks. This follows from the following lemma.

Lemma 6.10. Let $t, s \geqslant 0$ and $\mu, \lambda$ be bipartitions. If $E_{\Theta_{t}(\mu)}$ and $E_{\Theta_{s}(\lambda)}$ are in the same $\ell$-block then $\left|\mu, Q_{t}\right\rangle_{2 d}$ and $\left|\lambda, Q_{s}\right\rangle_{2 d}$ have the same weight for the action of $\mathfrak{g}_{2 d}^{\prime}$.

Proof. Recall that if $f$ is even (resp. odd), two unipotent characters of $G_{n}$ lie in the same $\ell$-block if and only if the corresponding symbols have the same $d$-cocore (resp. $d$-core), see 66.3 .4 . Therefore it is enough to show that adding/removing $d$-cohooks (resp. $d$-hooks) does not affect the weight of $\left|\mu, Q_{t}\right\rangle_{2 d}$ for the action of $\mathfrak{g}_{2 d}^{\prime}$.

Write $Q_{t}=\left(Q_{1}, Q_{2}\right)$ and $\mu=\left(\mu^{1}, \mu^{2}\right)$. Using Proposition 2.2 and (2.4) one can compute the weight of $\left|\mu, Q_{t}\right\rangle_{2 d}$ from the $\beta$-set $\beta_{0}(\mu)$. For $i \in I_{2 d}$ and $N$ large enough, we have

$$
\begin{aligned}
\left\langle\operatorname{wt}\left(\left|\mu, Q_{t}\right\rangle_{2 d}\right), \alpha_{i}^{\vee}\right\rangle= & \left|\left\{\beta \in \beta_{0}\left(\mu^{1}\right), \beta \geqslant-N \mid q^{\beta} Q_{1} \equiv i \bmod \ell\right\}\right| \\
& -\left|\left\{\beta \in \beta_{0}\left(\mu^{1}\right), \beta \geqslant-N+1 \mid q^{\beta} Q_{1} \equiv q i \bmod \ell\right\}\right| \\
& +\left|\left\{\beta \in \beta_{0}\left(\mu^{2}\right), \beta \geqslant-N \mid q^{\beta} Q_{2} \equiv i \bmod \ell\right\}\right| \\
& -\left|\left\{\beta \in \beta_{0}\left(\mu^{2}\right), \beta \geqslant-N+1 \mid q^{\beta} Q_{2} \equiv q i \bmod \ell\right\}\right| .
\end{aligned}
$$

This expression becomes simpler when working with the charged $\beta$-sets used in the definition of $\Theta_{t}(\mu)$ in $\$ 6.3 .2$. Write $\Theta_{t}(\mu)=\{X, Y\}$ where $X=\beta_{t}\left(\mu^{1}\right)$ and $Y=$ 
$\beta_{-1-t}\left(\mu^{2}\right)$. Then by definition of $Q_{t}$ we have, for $N$ large enough

$$
\begin{aligned}
\left\langle\operatorname{wt}\left(\left|\mu, Q_{t}\right\rangle_{2 d}\right), \alpha_{i}^{\vee}\right\rangle= & \left|\left\{x \in X, x \geqslant-N \mid q^{x} \equiv(-1)^{t} i \bmod \ell\right\}\right| \\
& -\left|\left\{x \in X, x \geqslant-N+1 \mid q^{x} \equiv(-1)^{t} q i \bmod \ell\right\}\right| \\
& +\left|\left\{y \in Y, y \geqslant-N \mid q^{y} \equiv(-1)^{t+1} i \bmod \ell\right\}\right| \\
& -\left|\left\{y \in Y, y \geqslant-N+1 \mid q^{y} \equiv(-1)^{t+1} q i \bmod \ell\right\}\right| .
\end{aligned}
$$

If $f$ is odd, then $q^{d}=q^{f}=1$ in $\mathrm{k}$ and adding or removing a $d$-hook has the effect of removing $x$ from $X$ (resp. $Y$ ) and adding $x \pm d$ to $X$ (resp. $Y$ ), which does not change the congruence of $q^{x}$. In particular, this operation does not affect $\operatorname{wt}\left(\left|\mu, Q_{t}\right\rangle_{2 d}\right)$.

If $f$ is even, then $q^{d}=-1, q^{f}=1$ and adding or removing a $d$-cohook has the effect of removing $x$ from $X$ (resp. $Y$ ) and adding $x \pm d$ to $Y$ (resp. $x$ ). This changes $q^{x}$ to $q^{x \pm d} \equiv-q^{x}$. Therefore if $t^{\prime}=t \pm 1$ and $\Theta_{t^{\prime}}\left(\mu^{\prime}\right)$ is the symbol obtained from $\Theta_{t}(\mu)$ by adding or removing a $d$-cohook we have

$$
\left.\left.\operatorname{wt}\left(\left|\mu, Q_{t}\right\rangle_{2 d}\right), \alpha_{i}^{\vee}\right\rangle=\operatorname{wt}\left(\left|\mu^{\prime}, Q_{t^{\prime}}\right\rangle_{2 d}\right), \alpha_{-i}^{\vee}\right\rangle .
$$

In particular, symbols corresponding to unipotent characters in the same $\ell$-block have the same weight.

Notice that the lemma implies that the simple unipotent modules are weight vectors of the $\mathfrak{g}_{2 d}^{\prime}$-action. From this and the results in the previous section we deduce the expected result.

Theorem 6.11. Recall that $\ell$ and $q$ are odd, and $\ell \nmid q\left(q^{2}-1\right)$. The representation datum on $\mathscr{U}_{\mathrm{k}}$ yields a $\mathfrak{g}_{2 d}^{\prime}$-representation such that the map $d_{\mathscr{U}}:\left[\mathscr{U}_{K}\right] \rightarrow\left[\mathscr{U}_{\mathrm{k}}\right]$ intertwines the representations of $\mathfrak{g}_{\infty}$ and $\mathfrak{g}_{2 d}^{\prime}$. There is a $\mathfrak{g}_{2 d}^{\prime}$-module isomorphism $\bigoplus_{t \in \mathbb{N}} \mathbf{F}\left(Q_{t}\right)_{2 d} \stackrel{\sim}{\rightarrow}\left[\mathscr{U}_{\mathrm{k}}\right]$ sending $\left|\mu, Q_{t}\right\rangle_{2 d}$ to $d_{\mathscr{U}}\left(\left[E_{\Theta_{t}(\mu)}\right]\right)$. Further, the classes in $\left[\mathscr{U}_{\mathrm{k}}\right]$ of the simple unipotent modules are weight vectors for the $\mathfrak{g}_{2 d}^{\prime}$-action.

6.5.2. The $\mathfrak{g}_{2 d}$-representation on $\mathscr{U}_{\mathrm{k}}$ in the linear prime case. In this section we assume that $f$ is odd, so $f=d$. In that case the Kac-Moody algebra $\widetilde{\mathfrak{g}}_{2 d}$ associated with the quiver $I_{2 d}$ is isomorphic to $\left(\widehat{\mathfrak{s l}}_{d}\right)^{\oplus 2}$. As in the case of unitary groups, see 4.5.1, the action of $\mathfrak{g}_{2 d}^{\prime}$ on $\mathscr{U}_{\mathrm{k}}$ can be naturally extended to an action of an algebra $\mathfrak{g}_{2 d}$ lying between $\mathfrak{g}_{2 d}^{\prime}$ and $\widetilde{\mathfrak{g}}_{2 d}$.

Let $\widetilde{\mathrm{X}}_{2 d}$ and $\widetilde{\mathrm{X}}_{2 d}^{\vee}$ be the lattices corresponding to $\widetilde{\mathfrak{g}}_{2 d}$. Since $f$ is odd, $I_{2 d}$ is the disjoint union of two cyclic quivers. We choose $\alpha_{1}$ and $\alpha_{-1}$ to be the affine roots attached to each of these quivers. Then we have

$$
\widetilde{\mathrm{X}}_{2 d}=\mathrm{P}_{2 d} \oplus \mathbb{Z} \delta_{+} \oplus \mathbb{Z} \delta_{-}, \quad \widetilde{\mathrm{X}}_{2 d}^{\vee}=\mathrm{Q}_{2 d}^{\vee} \oplus \mathbb{Z} \partial_{+} \oplus \mathbb{Z} \partial_{-},
$$


with $\delta_{+}=\sum \alpha_{q^{j}}, \delta_{-}=\sum \alpha_{-q^{j}}, \partial_{+}=\Lambda_{1}^{\vee}$ and $\partial_{-}=\Lambda_{-1}^{\vee}$. We set $\partial=\partial_{+}+\partial_{-}$and $\delta=\left(\delta_{+}+\delta_{-}\right) / 2$. We define $\mathfrak{g}_{2 d}:=\mathfrak{g}_{2 d}^{\prime} \oplus \mathbb{C} \partial$ and we view it as the Kac-Moody algebra associated with the lattices

$$
\mathrm{X}_{2 d}:=\mathrm{P}_{2 d} \oplus \mathbb{Z} \delta \simeq \widetilde{\mathrm{X}}_{2 d} /\left(\delta_{+}-\delta_{-}\right), \quad \mathrm{X}_{2 d}^{\vee}:=\mathrm{Q}_{2 d}^{\vee} \oplus \mathbb{Z} \partial
$$

The pairing $\widetilde{\mathrm{X}}_{2 d}^{\vee} \times \widetilde{\mathrm{X}}_{2 d} \longrightarrow \mathbb{Z}$ induces in the obvious way a perfect pairing $\mathrm{X}_{2 d}^{\vee} \times \mathrm{X}_{2 d} \longrightarrow$ $\mathbb{Z}$.

For $t \in \mathbb{N}$, the Fock space $\mathbf{F}\left(Q_{t}\right)_{2 d}$ has a tensor product decomposition into level 1 Fock spaces

$$
\mathbf{F}\left(Q_{t}\right)_{2 d} \simeq \mathbf{F}\left((-q)^{t}\right)_{d} \otimes \mathbf{F}\left((-q)^{-1-t}\right)_{d} .
$$

Out of the charged Fock spaces $\left(\mathbf{F}\left((-q)^{t}\right)_{d}, t\right)$ and $\left(\mathbf{F}\left((-q)^{-1-t}\right)_{d},-1-t\right)$ and the isomorphism $\widetilde{\mathfrak{g}}_{2 d} \simeq\left(\widehat{\mathfrak{s l}}_{d}\right)^{\oplus 2}$ (which depends on the parity of $t$ ) we can therefore equip $\mathbf{F}\left(Q_{t}\right)_{2 d}$ with an action of $\widetilde{\mathfrak{g}}_{2 d}$ which in turn restricts to an action of $\mathfrak{g}_{2 d}$.

Recall that two unipotent characters are in the same $\ell$-block if and only if the corresponding symbols have the same $d$-core. In particular, the unipotent characters of a given unipotent block all lie in the same Harish-Chandra series. In addition, two unipotent characters lying in different Harish-Chandra series lie in different blocks. Consequently, for each $t \in \mathbb{N}$ we can form the category $\mathscr{U}_{\mathrm{k}, t}$ associated with the Harish-Chandra series labelled by $t$, yielding

$$
\mathscr{U}_{\mathrm{k}}=\bigoplus_{t \in \mathbb{N}} \mathscr{U}_{\mathrm{k}, t} \quad \text { with } \quad\left[\mathscr{U}_{\mathrm{k}}\right] \simeq \mathbf{F}\left(Q_{t}\right)_{2 d}
$$

Using the action of $\mathfrak{g}_{2 d}$ on $\mathbf{F}\left(Q_{t}\right)_{2 d}$ defined above we equip each [ $\left.\mathscr{U}_{\mathrm{k}, t}\right]$ with a structure of $\mathfrak{g}_{2 d^{-}}$-module which extends the structure of $\mathfrak{g}_{2 d^{\prime}}^{\prime}$-module defined in $\$ 6.5 .1$. The situation is completely similar to $\$ 4.6 .1$ and we get the following theorem.

Theorem 6.12. Recall that $\ell$ and $q$ are odd, and $\ell \nmid q\left(q^{2}-1\right)$. Assume that $f$ is odd. For each $t \in \mathbb{N}$, the Harish-Chandra induction and restriction functors yield a representation of $\mathfrak{g}_{2 d}$ on $\mathscr{U}_{\mathrm{k}, t}$ which categorifies $\mathbf{F}\left(Q_{t}\right)_{2 d}$.

6.5.3. Combinatorics of $d$-cohooks and d-cocores. We now concentrate on the unitary prime case, i.e., the case where $f$ is even, which is the most delicate. We wish to define an action of a (non derived) Kac-Moody algebra $\mathfrak{g}_{2 d}$ on $\left[\mathscr{U}_{\mathrm{k}}\right]$ which extends the action of $\mathfrak{g}_{2 d}^{\prime}$. Then, we will extend the grading of $\left[\mathscr{U}_{\mathrm{k}}\right]$ from $\mathrm{P}_{2 d}$ to $\mathrm{X}_{2 d}=\mathrm{P}_{2 d} \oplus \mathbb{Z} \delta / 2$. Before this, we must introduce some combinatorial tools related to symbols.

Let $\mu=\left(\mu^{1}, \mu^{2}\right)$ be a bipartition and $t \in \mathbb{Z}$. Consider the symbol $\Theta$ defined by

$$
\Theta=\{X, Y-d\}=\Theta_{t}(\mu)
$$


where $X=\beta_{t}\left(\mu^{1}\right)$ and $Y=\beta_{d-1-t}\left(\mu^{2}\right)$. With this notation, removing a $d$-cohook on $\Theta$ changes the pair $(X, Y)$ to

$$
(X \backslash\{x\}, Y \sqcup\{x\}) \quad \text { or } \quad(X \sqcup\{y-2 d\}, Y \backslash\{y\}) .
$$

We denote by $s=\left(s_{1}, \ldots, s_{2 d}\right)$ and $r=\left(r_{1}, \ldots, r_{2 d}\right)$ the $2 d$-core of the charged partitions $\left(\mu^{1}, t\right)$ and $\left(\mu^{2}, d-1-t\right)$ respectively, and by $\left(\mu^{1,1}, \ldots, \mu^{1,2 d}\right)$ and $\left(\mu^{2,1}, \ldots, \mu^{2,2 d}\right)$ their $2 d$-quotients. Hence, we have

$$
\begin{aligned}
\tau_{2 d}\left(\mu^{1}, t\right) & =\left(\mu^{1,1}, \ldots, \mu^{1,2 d}, s_{1}, \ldots, s_{2 d}\right), \\
\tau_{2 d}\left(\mu^{2}, d-1-t\right) & =\left(\mu^{2,1}, \ldots, \mu^{2,2 d}, r_{1}, \ldots, r_{2 d}\right) .
\end{aligned}
$$

Lemma 6.13. The symbol $\Theta$ is a d-cocore if and only if

(a) $\mu^{1}$ and $\mu^{2}$ are $2 d$-cores,

(b) $r_{p}-s_{p} \in\{0,1\}$ for all $p \in\{1, \ldots, 2 d\}$.

Proof. Let us write $X=\beta_{t}\left(\mu^{1}\right)$ and $Y=\beta_{d-1-t}\left(\mu^{2}\right)$. Then $\Theta$ is a $d$-cocore if and only if $X \subset Y \subset X+2 d$. In particular, we must have $X \subset X+2 d$ and $Y \subset Y+2 d$, which is equivalent to $\mu^{1}$ and $\mu^{2}$ being $2 d$-cores. In that case we have

$$
X=\bigsqcup_{p=1}^{2 d}\left(p-2 d+2 d \beta_{s_{p}}(\emptyset)\right) \quad \text { and } \quad Y=\bigsqcup_{p=1}^{2 d}\left(p-2 d+2 d \beta_{r_{p}}(\emptyset)\right) .
$$

Using the definition of the $\beta$-set of an empty partition we deduce that $X \subset Y$ if and only if $s_{p} \leqslant r_{p}$ for all $p$. For the same reason, we have $Y \subset X+2 d$ if and only if $r_{p} \leqslant s_{p}+1$ for all $p$. The combination of the two conditions gives

$$
r_{p}-s_{p} \in\{0,1\}, \text { for all } p \text {. }
$$

Lemma 6.14. Let $\Theta, \Theta^{\prime}$ be two symbols. The following assertions are equivalent:

(i) there exists a sequence of symbols $\Theta=\Theta_{0}, \Theta_{1}, \ldots, \Theta_{m}=\Theta^{\prime}$ where $\Theta_{i+1}$ is obtained from $\Theta_{i}$ by adding or removing a d-cohook,

(ii) $s+r=s^{\prime}+r^{\prime}$.

Proof. Let $\mu$ be the bipartition such that $\Theta=\Theta_{t}(\mu)$. We write

$$
\beta_{t}\left(\mu^{1}\right)=\bigsqcup_{p=1}^{2 d}\left(p-2 d+2 d \beta_{s_{p}}\left(\mu^{1, p}\right)\right) \quad \text { and } \quad \beta_{d-1-t}\left(\mu^{2}\right)=\bigsqcup_{p=1}^{2 d}\left(p-2 d+2 d \beta_{r_{p}}\left(\mu^{2, p}\right)\right) .
$$

Therefore adding or removing a $d$-cohook changes $\left(s_{p}, r_{p}\right)$ to $\left(s_{p}, r_{p}\right) \pm(1,-1)$ for some $p \in\{1, \ldots, 2 d\}$. This does not change $s+r$. 
Let $\Theta_{\circ}, \Theta_{\circ}^{\prime}$ be the $d$-cocore of $\Theta, \Theta^{\prime}$, and $\left(s_{\circ}, r_{\circ}\right),\left(s_{\circ}^{\prime}, r_{\circ}^{\prime}\right)$ be the corresponding $2 d$-cores. By the previous argument, we have

$$
s+r=s_{\circ}+r_{\circ}, \quad s^{\prime}+r^{\prime}=s_{\circ}^{\prime}+r_{\circ}^{\prime} .
$$

Now, assertion (i) is equivalent to $\Theta_{\circ}=\Theta_{\circ}^{\prime}$, which yields in particular

$$
s+r=s_{\circ}+r_{\circ}=s^{\prime}+r^{\prime} .
$$

Conversely, if we assume (ii), then $s_{\circ}+r_{\circ}=s_{\circ}^{\prime}+r_{\circ}^{\prime}$ which we can write

$$
r_{\circ}-s_{\circ}=r_{\circ}^{\prime}-s_{\circ}^{\prime}+2\left(r_{\circ}-r_{\circ}^{\prime}\right) .
$$

But by Lemma 6.13, both $r_{\circ}-s_{\circ}$ and $r_{\circ}^{\prime}-s_{\circ}^{\prime}$ are $2 d$-tuples of integers equal to 0 or 1. This forces $r_{\circ}=r_{\circ}^{\prime}$ and therefore $s_{\circ}=s_{\circ}^{\prime}$. Since $t_{\circ}=\sum_{p} s_{\circ, p}$, this determines $\Theta_{\circ}$ uniquely and therefore we have $\Theta_{\circ}=\Theta_{\circ}^{\prime}$.

6.5.4. The weight of a symbol. We are still working under the assumption that $f=2 d$ is even. In particular $q^{d}=-1$ in $\mathrm{k}$. Fix an integer $t \in \mathbb{Z}$ and set

$$
P_{t}=q^{c_{t}}=\left(q^{t},-q^{-1-t}\right), \quad c_{t}=(t, d-1-t) .
$$

In particular $Q_{t}=(-1)^{t} P_{t}$. Let $\mu$ be a bipartition and let $s$ and $r$ be the $2 d$ cores of the charged partitions $\left(\mu^{1}, t\right)$ and $\left(\mu^{2}, d-1-t\right)$. We consider the weight in $\mathrm{X}=\mathrm{P} \oplus \mathbb{Z} \delta / 2$ given by

$$
\begin{aligned}
\operatorname{wt}(\mu, t) & =\Lambda_{P_{t}}-\sum_{i} n_{i}\left(\mu, P_{t}\right) \alpha_{i}-\nabla(s, r) \delta, \\
\nabla(s, r) & =\Delta(t, 2 d)+\Delta(d-1-t, 2 d)+t / 2,
\end{aligned}
$$

where $\Delta(t, 2 d)$ is as in (2.3). For any $2 d$-tuple of integers $x=\left(x_{1}, \ldots, x_{2 d}\right)$, we define as in $\$ 2.3 .1$

$$
\pi_{x}=\sum_{p=1}^{2 d}\left(x_{p}-x_{p+1}\right) \Lambda_{q^{p}} .
$$

Then, we have

$$
\begin{aligned}
\mathrm{wt}(\mu, t) & \equiv \Lambda_{P_{t}}-\sum_{i} n_{i}\left(\mu, P_{t}\right) \alpha_{i}^{\mathrm{cl}} \bmod \mathbb{Z} \delta \\
& \equiv \pi_{s+r}+2 \Lambda_{1} \bmod \mathbb{Z} \delta .
\end{aligned}
$$

The effect on the weight of removing a $d$-cohook is studied in the following lemma.

Lemma 6.15. Let $t, t^{\prime}$ be integers and $\mu, \mu^{\prime}$ be bipartitions. Set $\Theta=\Theta_{t}(\mu)$ and $\Theta^{\prime}=\Theta_{t^{\prime}}\left(\mu^{\prime}\right)$. If $\Theta^{\prime}$ is obtained from $\Theta$ by removing a $d$-cohook, then $\operatorname{wt}(\mu, t)=$ $\operatorname{wt}\left(\mu^{\prime}, t^{\prime}\right)-\delta / 2$. 
Proof. By (2.4), we have

$$
\begin{aligned}
\operatorname{wt}(\mu, t) & =\operatorname{wt}\left(\left|\mu^{1}, t\right\rangle_{2 d}\right)+\operatorname{wt}\left(\left|\mu^{2}, d-1-t\right\rangle_{2 d}\right)+(\Delta(t, 2 d)+\Delta(d-1-t, 2 d)-\nabla(s, r)) \delta \\
& =\operatorname{wt}\left(\left|\mu^{1}, t\right\rangle_{2 d}\right)+\operatorname{wt}\left(\left|\mu^{2}, d-1-t\right\rangle_{2 d}\right)-t \delta / 2 \\
& =\pi_{s+r}+2 \Lambda_{1}-\left(w_{2 d}\left(\mu^{1}\right)+w_{2 d}\left(\mu^{2}\right)+\Delta(s, 1)+\Delta(r, 1)+t / 2\right) \delta .
\end{aligned}
$$

where we used the formulas in $\$ 2.3 .1$. Therefore we must study the effect of removing a $d$-cohook on the integer

$$
w_{2 d}\left(\mu^{1}\right)+w_{2 d}\left(\mu^{2}\right)+\Delta(s, 1)+\Delta(r, 1)+t / 2 .
$$

Note that by Lemma 6.14, removing a $d$-cohook has no effect on $s+r$.

First, assume that there is an integer $x \in \beta_{t}\left(\mu^{1}\right)$ such that $x \notin \beta_{d-1-t}\left(\mu^{2}\right)$ and write

$$
\beta_{t^{\prime}}\left(\mu^{\prime 1}\right)=\beta_{t}\left(\mu^{1}\right) \backslash\{x\}, \quad \beta_{d-1-t^{\prime}}\left(\mu^{\prime 2}\right)=\beta_{d-1-t}\left(\mu^{2}\right) \sqcup\{x\} .
$$

Fix $p \in\{1, \ldots, 2 d\}$ and $z \in \beta_{s_{p}}\left(\mu^{1, p}\right)$ such that

$$
x=p-2 d+2 d z .
$$

Fix $u_{0}$ such that $z=z_{u_{0}}$ where $\beta_{s_{p}}\left(\mu^{1, p}\right)=\left\{z_{1}>z_{2}>\cdots\right\}$. We have

$$
\left|\mu^{1, p}\right|=\sum_{u \geqslant 1}\left(z_{u}+u-1-s_{p}\right) \text {. }
$$

We also have

$$
\begin{aligned}
\left|\mu^{\prime 1, p}\right|-\left|\mu^{1, p}\right| & =\sum_{u \geqslant 1}\left(z_{u}^{\prime}+u-1-s_{p}+1\right)-\sum_{u \geqslant 1}\left(z_{u}+u-1-s_{p}\right) \\
& =\sum_{1 \leq u<u_{0}}\left(z_{u}+u-s_{p}\right)+\sum_{u \geqslant u_{0}}\left(z_{u+1}+u-s_{p}\right)-\sum_{u \geqslant 1}\left(z_{u}+u-1-s_{p}\right) \\
& =s_{p}-z .
\end{aligned}
$$

We deduce that we have

$$
w_{2 d}\left(\mu^{\prime 1}\right)=w_{2 d}\left(\mu^{1}\right)+s_{p}-z .
$$

A similar computation yields

$$
w_{2 d}\left(\mu^{2}\right)=w_{2 d}\left(\mu^{\prime 2}\right)+r_{p}^{\prime}-z=w_{2 d}\left(\mu^{\prime 2}\right)+r_{p}+1-z .
$$

We deduce that

$$
w_{2 d}\left(\mu^{\prime 1}\right)+w_{2 d}\left(\mu^{\prime 2}\right)=w_{2 d}\left(\mu^{1}\right)+w_{2 d}\left(\mu^{2}\right)+s_{p}-r_{p}-1 .
$$

Since we have removed the element $x$ from $\beta_{t}\left(\mu^{1}\right)$, we have $t^{\prime}=t-1$.

Next, for any integers $v, e$ with $e \geqslant 1$, we have

$$
\Delta(v, e)-\Delta(v-1, e)=\left\lfloor\frac{v-1}{e}\right\rfloor .
$$


We deduce that

$$
\Delta\left(s^{\prime}, 1\right)-\Delta(s, 1)=1-s_{p}, \quad \Delta\left(r^{\prime}, 1\right)-\Delta(r, 1)=r_{p} .
$$

In particular, the value of

$$
w_{2 d}\left(\mu^{1}\right)+w_{2 d}\left(\mu^{2}\right)+\Delta(s, 1)+\Delta(r, 1)+t / 2
$$

decreases by $1 / 2$.

Now assume that there is an integer $y \in \beta_{d-1-t}\left(\mu^{2}\right)$ such that $y-2 d \notin \beta_{t}\left(\mu^{1}\right)$ and let us write

$$
\beta_{t^{\prime}}\left(\mu^{\prime 1}\right)=\beta_{t}\left(\mu^{1}\right) \sqcup\{y-2 d\}, \quad \beta_{d-1-t^{\prime}}\left(\mu^{\prime 2}\right)=\beta_{d-1-t}\left(\mu^{2}\right) \backslash\{y\} .
$$

Fix $p \in\{1, \ldots, 2 d\}$ and $z \in \beta_{r_{p}}\left(\mu^{2, p}\right)$ such that

$$
y=p-2 d+2 d z \text {. }
$$

The same computation as above gives

$$
w_{2 d}\left(\mu^{\prime 2}\right)=w_{2 d}\left(\mu^{2}\right)+r_{p}-z, \quad w_{2 d}\left(\mu^{1}\right)=w_{2 d}\left(\mu^{\prime 1}\right)+\left(s_{p}+1\right)-(z-1),
$$

from which we deduce that

$$
w_{2 d}\left(\mu^{\prime 1}\right)+w_{2 d}\left(\mu^{\prime 2}\right)=w_{2 d}\left(\mu^{1}\right)+w_{2 d}\left(\mu^{2}\right)+r_{p}-s_{p}-2 .
$$

Since $t^{\prime}=t+1$, the value of

$$
w_{2 d}\left(\mu^{1}\right)+w_{2 d}\left(\mu^{2}\right)+\Delta(s, 1)+\Delta(r, 1)+t / 2
$$

decreases by $1 / 2$.

6.5.5. The $\mathfrak{g}_{2 d}$-representation on $\mathscr{U}_{\mathrm{k}}$ in the unitary case. We now have all the tools to extend the action of $\mathfrak{g}_{2 d}^{\prime}$ to $\mathfrak{g}_{2 d}$ on $\mathscr{U}_{\mathrm{k}}$. Recall that we assume that $f$ is even, so $f=2 d$. Hence $I_{2 d}$ is a cyclic quiver of size $2 d$ and we have an isomorphism $\widetilde{\mathfrak{s l}}_{f} \stackrel{\sim}{\rightarrow} \mathfrak{g}_{2 d}^{\prime}$ sending $\alpha_{k}^{\vee}$ to $\alpha_{q^{k}}^{\vee}$. Let $\mathfrak{g}_{2 d}$ be the Kac-Moody algebra associated with the quiver $I_{2 d}$, i.e., we have $\widehat{\mathfrak{s l}}_{f} \simeq \mathfrak{g}_{2 d}$, and set $\mathrm{X}_{2 d}=\mathrm{P}_{2 d} \oplus \mathbb{Z} \delta / 2$.

Let $\sigma$ be the automorphism of the Lie algebra $\mathfrak{g}_{2 d}$ given by the quiver automorphism of $I_{2 d}$ such that $i \mapsto-i$. In particular, we have $\sigma\left(\alpha_{i}^{\vee}\right)=\alpha_{-i}^{\vee}$.

Definition 6.16. We define the weight of a symbol by $\operatorname{wt}\left(\Theta_{t}(\mu)\right)=\sigma_{*}^{t}(\operatorname{wt}(\mu, t))$, where $\mathrm{wt}(\mu, t)$ is as in (6.6).

We can now prove the following.

Proposition 6.17. Let $\mu$ be a bipartition and $t \in \mathbb{Z}$. Let $\Theta, \Theta^{\prime}$ be symbols.

(a) $\operatorname{wt}\left(\Theta_{t}(\mu)\right) \equiv \operatorname{wt}\left(\left|\mu, Q_{t}\right\rangle_{2 d}\right) \bmod \mathbb{Z} \delta$,

(b) if $\Theta^{\prime}$ is obtained from $\Theta$ by removing a d-hook, then $\operatorname{wt}(\Theta)=\sigma_{*} \operatorname{wt}\left(\Theta^{\prime}\right)-\delta / 2$,

(c) $E_{\Theta}, E_{\Theta^{\prime}}$ belong to the same $\ell$-block if and only if we have $\operatorname{wt}(\Theta)=\operatorname{wt}\left(\Theta^{\prime}\right)$. 
Proof. We have $Q_{t}=(-1)^{t} P_{t}$. Further,

$$
\begin{aligned}
\operatorname{wt}(\mu, t) & \equiv \Lambda_{P_{t}}-\sum_{i} n_{i}\left(\mu, P_{t}\right) \alpha_{i}^{\mathrm{cl}} \bmod \mathbb{Z} \delta, \\
\mathrm{wt}\left(\left|\mu, Q_{t}\right\rangle_{2 d}\right) & \equiv \Lambda_{Q_{t}}-\sum_{i} n_{i}\left(\mu, Q_{t}\right) \alpha_{i}^{\mathrm{cl}} \bmod \mathbb{Z} \delta .
\end{aligned}
$$

This implies part (a) because

$$
n_{(-1)^{t} i}\left(\mu, P_{t}\right)=n_{i}\left(\mu,(-1)^{t} P_{t}\right)=n_{i}\left(\mu, Q_{t}\right) .
$$

Part (b) follows from Lemma 6.15.

Now, we prove part (c). Recall that the representations $E_{\Theta}$ and $E_{\Theta^{\prime}}$ are in the same $\ell$-block if and only if $\Theta$ and $\Theta^{\prime}$ have the same rank and the same $d$-cocore $\Theta_{\circ}$, see 6.3 .4 .

Assume that this is the case. Since the number of $d$-cohooks one needs to add to $\Theta_{\circ}$ to obtain $\Theta$ and $\Theta^{\prime}$ depends only on the rank of the symbols, we deduce from Lemma 6.15 that $\Theta$ and $\Theta^{\prime}$ have the same weight.

Conversely, assume now that $\operatorname{wt}(\Theta)=\operatorname{wt}\left(\Theta^{\prime}\right)$. Then (6.7) implies that

$$
\pi_{s+r}=\pi_{s^{\prime}+r^{\prime}} .
$$

By definition of $s, r, s^{\prime}$ and $r^{\prime}$ we have

$$
\sum_{p} s_{p}=t, \quad \sum_{p} r_{p}=d-1-t, \quad \sum_{p} s_{p}^{\prime}=t^{\prime}, \quad \sum_{p} r_{p}^{\prime}=d-1-t^{\prime}
$$

hence

$$
\sum_{p}\left(s_{p}+r_{p}\right)=\sum_{p}\left(s_{p}^{\prime}+r_{p}^{\prime}\right)=d-1
$$

From (6.8) and (6.9), we deduce that the $2 d$-tuples $s+r$ and $s^{\prime}+r^{\prime}$ are equal. Hence, by Lemma 6.14, the symbols $\Theta$ and $\Theta^{\prime}$ have the same $d$-cocore $\Theta_{\circ}$. Let $w$ and $w^{\prime}$ be the number of $d$-cohooks we need to add to $\Theta_{\circ}$ to obtain $\Theta$ and $\Theta^{\prime}$ respectively. Since $\sigma_{*}(\delta)=\delta$, by part (b) we have

$$
\begin{aligned}
\operatorname{wt}(\Theta) & =\left(\sigma_{*}\right)^{w} \operatorname{wt}\left(\Theta_{\circ}\right)-w \delta / 2, \\
\operatorname{wt}\left(\Theta^{\prime}\right) & =\left(\sigma_{*}\right)^{w^{\prime}} \operatorname{wt}\left(\Theta_{\circ}\right)-w^{\prime} \delta / 2 .
\end{aligned}
$$

Since $\sigma_{*}(\delta)=\delta$ and $\operatorname{wt}(\Theta)=\operatorname{wt}\left(\Theta^{\prime}\right)$, we deduce that $w=w^{\prime}$. In particular, the symbols $\Theta$ and $\Theta^{\prime}$ have the same rank, which shows that the corresponding representations lie in the same $\ell$-block.

We have proved the following refinement of Theorem 6.11. 
Theorem 6.18. Recall that $\ell$ and $q$ are odd, and $\ell \nmid q\left(q^{2}-1\right)$. Assume that $f$ is even. The representation datum on $\mathscr{U}_{\mathrm{k}}$ gives a $\mathfrak{g}_{2 d^{-}}$representation yielding a $\mathfrak{g}_{2 d^{-}}$ module isomorphism $\bigoplus_{t \in \mathbb{N}} \mathbf{F}\left(Q_{t}\right)_{2 d} \stackrel{\sim}{\rightarrow}\left[\mathscr{U}_{\mathrm{k}}\right]$ such that $\left|\mu, Q_{t}\right\rangle_{2 d} \mapsto d_{\mathscr{U}}\left(\left[E_{\Theta_{t}(\mu)}\right]\right)$. The weights of the $\mathfrak{g}_{2 d}$-module $\left[\mathscr{U}_{\mathrm{k}}\right]$ belong to the lattice $\mathrm{X}_{2 d}$. The weight of $d_{\mathscr{U}}\left(\left[E_{\Theta_{t}(\mu)}\right]\right)$ is equal to $\mathrm{wt}\left(\Theta_{t}(\mu)\right)$. The classes in $\left[\mathscr{U}_{\mathrm{k}}\right]$ of the simple unipotent modules are weight vectors. Two simple unipotent modules belong to the same block if and only if their classes have the same weight in $\mathrm{X}_{2 d}$.

6.5.6. Determination of the ramified Hecke algebras. As in the case of finite unitary groups, see 4.7 .2 , we can invoke Proposition 1.5 to show that every ramified Hecke algebra associated to a weakly cuspidal module is indeed a Hecke algebra, whose parameters can be read off from the weight of the cuspidal module.

Corollary 6.19. Let $D$ be a weakly cuspidal $\mathrm{k} G_{r}$-module. Then

(a) the weight of the class $[D]$ with respect to the $\mathfrak{g}_{2 d^{\prime}}^{\prime}$ action on $\left[\mathscr{U}_{\mathrm{k}}\right]$ is of the form $\Lambda_{Q}$ for some element $Q=\left(Q_{1}, Q_{2}\right)$ in $I_{2 d}^{2}$,

(b) for each $m \geqslant 0$ and $n=r+2 m$, the $\operatorname{map} \phi_{\mathrm{k}, m}: \mathbf{H}_{\mathrm{k}, m}^{q^{2}} \longrightarrow \operatorname{End}_{\mathrm{k} G_{n}}\left(F^{m}\right)^{\mathrm{op}}$ factors to an algebra isomorphism $\mathbf{H}_{\mathrm{k}, m}^{Q ; q^{2}} \stackrel{\sim}{\longrightarrow} \mathscr{H}\left(\mathrm{k} G_{n}, D\right)$.

In order to apply this corollary efficiently, we must determine explicitly the weakly cuspidal modules and their weights. In the linear prime case, this is known by [39]. In the unitary prime case, by Theorem 6.18, the weakly cuspidal modules form a basis of the subspace of highest weight vectors of the representation of $\mathfrak{g}_{2 d}^{\prime}$ on $\left[\mathscr{U}_{\mathrm{k}}\right]$, hence all ramified Hecke algebras can be explicitly computed. If, moreover, we assume that the decomposition matrix is unitriangular with respect to the $a$-function, see Conjecture 6.21 below, then the weakly cuspidal modules and their weights can also be read off from the crystal isomorphism in Theorem 6.22 below.

More precisely, any weakly cuspidal module is of the form $D=D_{\Theta}$ for some symbol $\Theta=\Theta_{t}(\mu)$. The parameter $Q$ can be explicitly computed from $\mu$ and $t$ according to the rule

$$
\begin{aligned}
\operatorname{wt}\left(D_{\Theta}\right) & \equiv \Lambda_{Q_{t}}-\sum_{i} n_{i}\left(\mu, Q_{t}\right) \alpha_{i}^{\mathrm{cl}} \bmod \mathbb{Z} \delta \\
& \equiv \Lambda_{Q_{1}}+\Lambda_{Q_{2}} \bmod \mathbb{Z} \delta
\end{aligned}
$$

Since the isomorphism in Proposition 6.9 identifies the set

$$
\left\{\left[D_{\Theta_{t}(\mu)}\right] ; \mu \in \mathscr{P}^{2}, D_{\Theta_{t}(\mu)} \text { is weakly cuspidal }\right\}
$$

with a basis of the space of highest weight vectors

$$
\left\{x \in \mathbf{F}\left(Q_{t}\right)_{2 d} ; E_{i}(x)=0, \forall i=1,2, \ldots, 2 d\right\},
$$


the weakly cuspidal modules correspond precisely to the elements in the crystal $B\left(s_{t}\right)_{2 d}$ which are killed by the operators $\widetilde{E}_{1}, \ldots, \widetilde{E}_{2 d}$, with the notation in 66.7 .3 below. These elements have been computed recently in [43, thm. 5.9] in terms of bipartitions $\mu$ whose abacus is totally $2 d$-periodic. $\mathrm{N}$

\subsection{Derived equivalences.}

Blocks of $\mathscr{U}_{\mathrm{k}}$ are parametrized by pairs $(\Theta, w)$ where $\Theta$ is a $d$-core (resp. a $d$ cocore) when $f$ is odd (resp. $f$ is even) and $w$ is a non-negative integer called the degree of the block. The unipotent characters lying in $B_{\Theta, w}$ are parametrized by the symbols which are obtained from $\Theta$ by adding $w$ successive $d$-hooks (resp. $d$-cohooks) if $f$ is odd (resp. $f$ is even).

Our categorification theorems (see Theorem 6.18 and 6.12) show that blocks of $\mathscr{U}_{\mathrm{k}}$ and $\mathscr{U}_{\mathrm{k}, t}$ correspond to weight spaces for the action of the Kac-Moody algebra $\mathfrak{g}_{2 d}$. Using the work of Chuang-Rouquier [10] (see also Theorem 1.11) we obtain derived equivalences between blocks of $\mathscr{U}_{\mathrm{k}}$ which are in the same orbit under the action of the affine Weyl group $W_{2 d}$.

If $\ell$ is a linear prime (i.e. $f$ is odd), the situation is similar to the case of finite unitary groups. The $d$-cores, which are formed by pairs of partitions which are both $d$-cores, form a single orbit under the action of $W_{2 d}$. In particular, orbits of blocks of $\mathscr{U}_{\mathrm{k}, t}$ under $W_{2 d}$ are parametrized by their degree. Let $w \in \mathbb{N}$. Livesey showed in [51, thm. 7.1] how to construct a good block of $\mathscr{U}_{\mathrm{k}, t}$ of degree $w$ which is derived equivalent to its Brauer correspondent. We deduce that Broué's abelian defect group conjecture holds for groups of type $B C$ at odd linear primes.

Theorem 6.20. Recall that $\ell$ and $q$ are odd. Assume that the order $f$ of $q$ modulo $\ell$ is even. Let $B$ be a unipotent block of $G_{n}$ over $\mathrm{k}$ or $\mathscr{O}$, and $D$ be a defect group of $B$. If $D$ is abelian, then $B$ is derived equivalent to its Brauer correspondent in $N_{G_{n}}(D)$.

\subsection{The crystal of $\mathscr{U}_{\mathrm{k}}$.}

As in the case of finite unitary groups, see 4.7 , our goal is to compare the crystal of the categorical representation on $\mathscr{U}_{\mathrm{k}}$ with the crystal of the $\mathfrak{g}_{2 d^{-}}$-module $\bigoplus_{t} \mathbf{F}\left(Q_{t}\right)_{2 d}$. In order to get an explicit crystal isomorphism, we will assume that the $\ell$-decomposition matrices of unipotent blocks are unitriangular with respect to the $a$-function. This gives a parametrization of the simple objects of $\mathscr{U}_{\mathrm{k}}$ and a combinatorial way (yet conjectural) to compute the (weak) Harish-Chandra branching graph. 
Throughout this section we will assume that $\ell$ is a unitary prime, which means that the order $f$ of $q$ modulo $\ell$ is even. We will write $f=2 d$.

6.7.1. Ordering symbols. For $t \geqslant 0$, we define the charge

$$
s_{t}= \begin{cases}(t, d-1-t) & \text { if } t \text { is even } \\ (t-d,-1-t) & \text { if } t \text { is odd }\end{cases}
$$

The symbol associated with a bipartition $\mu$ is $\Theta=\Theta_{t}(\mu)=\left\{\beta_{t}\left(\mu^{1}\right), \beta_{-1-t}\left(\mu^{2}\right)\right\}$. As in [33, $\{5.5 .10]$, we can consider the union of the two $\beta$-sets $\beta_{t}\left(\mu^{1}\right)$ and $\beta_{-1-t}\left(\mu^{2}\right)$ (allowing multiplicities) and write their elements in non-increasing order

$$
\kappa_{m_{t}}(\mu):=\left(\kappa_{1} \geqslant \kappa_{2} \geqslant \kappa_{3} \geqslant \cdots\right)
$$

where the parameter $m_{t}$ is given by

$$
m_{t}=(t,-1-t) .
$$

We can check that $\kappa_{i}=\lfloor i / 2\rfloor$ for $i$ large enough. Then, we define the $a$-value of the symbol $\Theta$ by

$$
a\left(\Theta_{t}(\mu)\right)=a(\Theta):=\sum_{i=1}^{\infty}(i-1)\left(\kappa_{i}-\left\lfloor\frac{i}{2}\right\rfloor\right) .
$$

This differs slightly from the $a$-value $a^{\left(m_{t}, 1\right)}(\mu)$ defined in [33] : from [33, prop. 5.5.11] we have $a\left(\Theta_{t}(\mu)\right)=a^{\left(m_{t}, 1\right)}(\mu)+a\left(\Theta_{t}(\emptyset)\right)$.

On the set of symbols in the same Harish-Chandra series and of the same rank we define as in [33, §5.7.5] a partial order by

$$
\Theta_{t}(\mu) \preccurlyeq \Theta_{t}(\lambda) \Longleftrightarrow \mu \ll_{m_{t}} \lambda \Longleftrightarrow \lambda=\mu \text { or } \kappa_{m_{t}}(\lambda)<\kappa_{m_{t}}(\mu) .
$$

We deduce from [33, prop. 5.7.7] that

$$
\Theta_{t}(\mu) \prec \Theta_{t}(\lambda) \Longrightarrow a\left(\Theta_{t}(\mu)\right)<a\left(\Theta_{t}(\lambda)\right) .
$$

6.7.2. Parametrization of unipotent modules. By Proposition 3.4 we know that when $\ell$ is odd, there are as many unipotent $\mathrm{k} G_{n}$-modules as symbols of odd defect and rank $n$. However there is no known natural parametrization of these modules in terms of symbols unless the decomposition matrix has unitriangular shape. This property has been proved when $f$ is odd [39], i.e., when $\ell$ is a linear prime, or when $f$ and $n$ are small. Geck conjectured in [30, conj. 3.4] that it should always hold.

Conjecture 6.21 (Geck). Assume $\ell$ is odd. There exists a parametrization

$$
\operatorname{Irr}\left(\mathscr{U}_{\mathrm{k}}\right)=\left\{\left[D_{\Theta}\right] \mid \lambda \in \mathscr{S}_{\text {odd }}\right\}
$$


of the simple unipotent modules and an ordering $\leqslant$ on symbols such that the decomposition map satisfies the following unitriangularity property

$$
d_{\mathscr{U}}\left(\left[E_{\Theta}\right]\right) \in\left[D_{\Theta}\right]+\sum_{\Theta^{\prime}<\Theta} \mathbb{Z}\left[D_{\Theta^{\prime}}\right] .
$$

Moreover, the ordering $\leqslant$ can be chosen to be compatible with the a-function so that $\Theta^{\prime} \leqslant \Theta$ implies $a\left(\Theta^{\prime}\right) \leqslant a(\Theta)$.

This conjecture is expected to hold for any finite reductive group as long as $\ell$ is a very good prime. In that case the ordering $\leqslant$ should be induced from the partial order on families of unipotent characters defined by Lusztig.

6.7.3. Comparison of the crystals. Recall that to any categorical representation one can associate a perfect basis, and hence an abstract crystal, see Proposition 1.9. In $\$ 6.5 .1$ we constructed a categorical representation of $\mathfrak{g}_{2 d}^{\prime}$ on the category $\mathscr{U}_{\mathrm{k}}$ of unipotent representations over $\mathrm{k}$. From this categorical representation we get an abstract crystal $B\left(\mathscr{U}_{\mathrm{k}}\right)=\left(\operatorname{Irr}\left(\mathscr{U}_{\mathrm{k}}\right), \widetilde{E}_{i}, \widetilde{F}_{i}\right)$. As for finite unitary groups, this crystal is related to the (weak) Harish-Chandra series. More precisely, the (uncolored) crystal graph associated with $B\left(\mathscr{U}_{\mathrm{k}}\right)$ coincides with the weak Harish-Chandra branching graph, and its connected components with the weak Harish-Chandra series. See 4.7 .1 for more details.

Recall from Theorem 6.11 that $\left[\mathscr{U}_{\mathrm{k}}\right]$ is isomorphic to a direct sum of Fock spaces $\mathbf{F}\left(Q_{t}\right)_{2 d}$ where $t$ runs over $\mathbb{N}$ and $Q_{t}=\left(Q_{1}, Q_{2}\right)=\left((-q)^{t},(-q)^{-1-t}\right)$. Recall from (6.10) that we have defined the charge $s_{t}=\left(s_{1}, s_{2}\right)$ by

$$
s_{t}= \begin{cases}(t, d-1-t) & \text { if } t \text { is even } \\ (t-d,-1-t) & \text { if } t \text { is odd }\end{cases}
$$

and $m_{t}=(t,-1-t)$. Since $q^{d}=-1$ in $\mathrm{k}$, we have $Q_{p}=q^{2 s_{p}}$ for each $p=1,2$. In other words $s_{t}$ is a charge for $\mathbf{F}\left(Q_{t}\right)_{2 d}$ with respect to $q$. Let $B\left(s_{t}\right)_{2 d}$ be the corresponding abstract crystal of $\mathbf{F}\left(Q_{t}\right)_{2 d}$, with the canonical labeling $B\left(s_{t}\right)_{2 d}=$ $\left\{b\left(\mu, s_{t}\right) \mid \mu \in \mathscr{P}^{2}, t \in \mathbb{N}\right\}$. Finally, we set $B_{2 d}=\bigsqcup_{t \in \mathbb{N}} B\left(s_{t}\right)_{2 d}$.

Under the assumption that the decomposition matrix is unitriangular with respect to the $a$-function, we can compare the crystal $B_{2 d}$ with the abstract crystal $B\left(\mathscr{U}_{\mathrm{k}}\right)$ coming from the categorical action of $\widehat{\mathfrak{s l}}_{2 d}$ on $\mathscr{U}_{\mathrm{k}}$.

Theorem 6.22. Recall that $\ell, q$ are odd and $f$ is even. If Conjecture 6.21 holds, then the map $b\left(\mu, s_{t}\right) \mapsto\left[D_{\Theta_{t}(\mu)}\right]$ is a crystal isomorphism $B_{2 d} \stackrel{\sim}{\rightarrow} B\left(\mathscr{U}_{\mathrm{k}}\right)$. 
Proof. Let $t \in \mathbb{Z}$ and $\mu$ be a bipartition. By [33, thm. 6.6.12] the Uglov basis satisfies

$$
b\left(\mu, s_{t}\right) \in\left|\mu, s_{t}\right\rangle+\sum_{\substack{\lambda \neq \mu \\ \lambda \ll m_{t}}} \mathbb{Z}\left|\lambda, s_{t}\right\rangle .
$$

Let $\phi:\left|\mu, s_{t}\right\rangle \mapsto d_{\mathscr{U}}\left(\left[E_{\Theta_{t}(\mu)}\right]\right)$ be the morphism of $\mathfrak{g}_{2 d^{\prime}}^{\prime}$-modules studied in $\$ 6.5 .1$. By the previous formula and the definition of $\preccurlyeq$ we have

$$
\phi\left(b\left(\mu, s_{t}\right)\right) \in d_{\mathscr{U}}\left(\left[E_{\Theta_{t}(\mu)}\right]\right)+\sum_{\substack{\Theta^{\prime} \neq \Theta_{t}(\mu) \\ \Theta^{\prime} \preccurlyeq \Theta_{t}(\mu)}} \mathbb{Z} d_{\mathscr{U}}\left(\left[E_{\Theta^{\prime}}\right]\right) .
$$

Let $n$ be the rank of the symbol $\Theta_{t}(\mu)$. If Conjecture 6.21 holds, then we can choose a total order $\leqslant$ on symbols of rank $n$ and odd defect, compatible with the $a$-function and for which the decomposition map $d_{\mathscr{U}}$ is unitriangular. In particular, using (6.11) we have

$$
\Theta^{\prime} \neq \Theta_{t}(\mu) \text { and } \Theta^{\prime} \preccurlyeq \Theta_{t}(\mu) \Longrightarrow a\left(\Theta^{\prime}\right)<a\left(\Theta_{t}(\mu)\right) \Longrightarrow \Theta^{\prime} \leqslant \Theta_{t}(\mu) .
$$

This, together with the previous formula shows that

$$
\phi\left(b\left(\mu, s_{t}\right)\right) \in\left[D_{\Theta_{t}(\mu)}\right]+\sum_{\substack{\Theta^{\prime} \neq \Theta_{t}(\mu) \\ \Theta^{\prime} \leqslant \Theta_{t}(\mu)}} \mathbb{Z}\left[D_{\Theta^{\prime}}\right]
$$

and we can invoke Proposition 1.10 to conclude.

Remark 6.23. Note that the definition of $s_{t}$ makes sense for negative integers $t$ as well. Then $b\left(\mu, s_{t}\right) \longmapsto b\left(\mu^{\dagger}, s_{-1-t}\right)$ induces an isomorphism of crystals. This is a particular case of changing the charge $(a, b)$ to $(b-d, a+d)$.

\section{REFERENCES}

[1] Berenstein, A., Kazhdan, D., Geometric and unipotent crystals. II. From unipotent bicrystals to crystal bases. Quantum groups, 13-88, Contemp. Math., 433, Amer. Math. Soc., Providence, RI, 2007.

[2] Bonnafé, C., Michel, J., Computational proof of the Mackey formula for q>2, J. Algebra 327 (2011), 506-526.

[3] Bonnafé, C., Rouquier, R., Catégories dérivées et variétés de Deligne-Lusztig, Publ. Math. I.H.E.S., 97 (2003), 1-59.

[4] Broué, M., Malle, G., Michel, J., Generic blocks of finite reductive groups. Représentations unipotentes génériques et blocs des groupes réductifs finis, Astérisque 212 (1993), 7-92.

[5] Broué, M., Michel, J., Blocs et séries de Lusztig dans un groupe réductif fini, J. Reine Angew. Math. 395 (1989), 56-67.

[6] Brundan, J., Dipper, R., Kleshchev, A., Quantum linear groups and representations of $\mathrm{GL}_{n}\left(\mathbb{F}_{q}\right)$. Mem. Amer. Math. Soc. 149 (2001). 
[7] Cabanes, M., Enguehard, M., On unipotent blocks and their ordinary characters., Invent. Math. 117 (1994), 149-164.

[8] Cabanes, M., Enguehard, M., Representation theory of finite reductive groups. New Mathematical Monographs, 1. Cambridge University Press, Cambridge, 2004.

[9] Carter, R. W., Finite groups of Lie type. Conjugacy classes and complex characters. WileyInterscience (1985).

[10] Chuang, J., Rouquier, R., Derived equivalences for symmetric groups and $\mathfrak{s l}_{2}$-categorification, Annals of Math. 167 (2008), 245-298.

[11] Curtis, C.W., Reiner, I., Methods of representation theory. Vol. I,II. Pure and Applied Mathematics. A Wiley-Interscience Publication. John Wiley \& Sons, Inc., New York, 1981, 1987.

[12] Digne, F., Michel, J., Representations of finite groups of Lie type, London Mathematical Society Student Texts, 2, Cambridge University Press, Cambridge, 1991.

[13] Dipper, R., On the decomposition numbers of the finite general linear groups I, Trans. Amer. Math. Soc. 290 (1985), 315-344.

[14] Dipper, R., On the decomposition numbers of the finite general linear groups II, Trans. Amer. Math. Soc. 292 (1985), 123-133.

[15] Dipper, R., James, G. D., The q-Schur algebra, Proc. Lond. Math. Soc. 59 (1989), 23-50.

[16] Dipper, R., Du, J., Harish-Chandra vertices and Steinberg's tensor product theorem for general linear groups, Proc. London Math. Soc. 75 (1997), 559-599.

[17] Dudas, O., Malle, G., Decomposition matrices for low-rank unitary groups, Proc. Lond. Math. Soc. 110 (2015), 1517-1557.

[18] Dudas, O., Varagnolo, M., Vasserot, E., Categorical actions on unipotent representations I. Finite unitary groups, arXiv:1509.03269.

[19] Dudas, O., Varagnolo, M., Vasserot, E., Categorical actions from Lusztig induction and restriction on finite linear groups, preprint 2015.

[20] Dunkl, C., Griffeth, S., Generalized Jack polynomials and the representation theory of rational Cherednik algebras, Selecta Math. (N.S.) 16 (2010), 791-818.

[21] Foda, O., Leclerc, B., Okado, M., Thibon, J.-Y., Welsh, T. A., Branching functions of $A_{n}^{(1)}$ and Jantzen-Seitz problem for Ariki-Koike algebras, Adv. Math. 141 (1999), 322-365.

[22] Fong, P., Srinivasan, B., The blocks of finite general linear and unitary groups, Invent. Math. 69 (1982), 109-153.

[23] Fong, P., Srinivasan, B., The blocks of finite classical groups, J. Reine Angew. Math. 396 (1989), 122-191.

[24] Fong, P., Srinivasan, B., Brauer trees in classical groups, J. Algebra 131 (1990), 179-225.

[25] Geck, M., An introduction to algebraic geometry and algebraic groups. Oxford Graduate Texts in Mathematics, 10. Oxford University Press, Oxford, 2003.

[26] Geck, M., On the decomposition numbers of the finite unitary groups in nondefining characteristic, Math. Z. 207 (1991), 83-89.

[27] Geck, M., Basic sets of Brauer characters of finite groups of Lie type. II, J. London Math. Soc. (2) 47 (1993), 255-268.

[28] Geck, M., Modular Harish-Chandra series, Hecke algebras and (generalized) q-Schur algebras. Modular representation theory of finite groups (Charlottesville, VA, 1998), 1-66, de Gruyter, Berlin, 2001.

[29] Geck, M., Hiss, G., Basic sets of Brauer characters of finite groups of Lie type, J. Reine Angew. Math. 418 (1991), 173-188. 
[30] Geck, M., Hiss, G., Modular representations of finite groups of Lie type in non-defining characteristic. In Finite reductive groups (Luminy, 1994), Progr. Math. 141, 195-249, Birkhäuser Boston, Boston, MA, 1997.

[31] Geck, M., Hiss, G., Malle, G., Cuspidal unipotent Brauer characters, J. Algebra 168 (1994), $182-220$.

[32] Geck, M., Hiss, G., Malle, G., Towards a classification of the irreducible representations in non-describing characteristic of a finite group of Lie type, Math. Z. 221 (1996), no. 3, 353-386.

[33] Geck, M., Jacon, N., Representations of Hecke algebras at roots of unity. Algebra and Applications, 15. Springer-Verlag London, Ltd., London, 2011.

[34] Geck, M., Pfeiffer, G., Characters of finite Coxeter groups and Iwahori-Hecke algebras. London Mathematical Society Monographs. New Series, 21. The Clarendon Press, Oxford University Press, New York, 2000.

[35] Gerber, T., Triple crystal action in Fock spaces, arXiv:1601.00581.

[36] Gerber, T., Hiss, G., Jacon, N., Harish-Chandra series in finite unitary groups and crystal graphs, Int. Math. Res. Not. 22 (2015), 12206-12250.

[37] Gerber, T., Hiss, G., Branching graphs for finite unitary groups in non-defining characteristic, arXiv:1502.01868v1.

[38] Gruber, J., Green vertex theory, Green correspondence, and Harish-Chandra induction, J. Algebra 186 (1996), 476-521.

[39] Gruber, J., Hiss, G., Decomposition numbers of finite classical groups for linear primes, J. Reine Angew. Math. 485 (1997), 55-91.

[40] Hiss, G., Regular and semisimple blocks of finite reductive groups, J . London Math. Soc. 41 (1990), 63-68.

[41] Hiss, G., Harish-Chandra series of Brauer characters in a finite group with a split BN-pair, J. London Math. Soc. (2) 48 (1993), 219-228.

[42] Howlett, R.B., Lehrer, G.I., Induced cuspidal representations and generalized Hecke rings, Invent. Math. 58 (1980), 37-64.

[43] Jacon, N., Lecouvey, C., A combinatorial decomposition of higher level Fock spaces, Osaka J. Math. 50 (2013), 897-920.

[44] Jimbo, M., Misra, K.C., Miwa, T., Okado, M., Combinatorics of representations of $U_{q}(\widehat{\mathfrak{g}}(n))$ at $q=0$, Comm. Math. Phys. 136 (1991), 543-566.

[45] Kac, V., Infinite dimensional Lie algebras, 3rd ed., Cambridge Univ. Press, 1990.

[46] Kang, S.J., Kashiwara, M., Categorification of highest weight modules via Khovanov-LaudaRouquier algebras, Invent. Math. 190 (2012), 699-742.

[47] Kang, S.J., Oh, S.J., Park, E., Perfect bases for integrable modules over generalized Kac-Moody algebras, Algebr. Represent. Theory 14 (2011), 571-587.

[48] Kashiwara, M., On crystal bases, Representations of Groups, Proceedings of the 1994 Annual Seminar of the Canadian Math. Soc. Ban 16 (1995) 155-197.

[49] Kashiwara, M., Biadjointness in cyclotomic Khovanov-Lauda-Rouquier algebras, Publ. Res. Inst. Math. Sci. 48 (2012), 501-524.

[50] Lascoux, A., Leclerc, B., Thibon, J.Y., Hecke algebras at roots of unity and crystal bases of quantum affine algebras, Comm. Math. Phys. 181 (1996), 205-263.

[51] Livesey, M., On Rouquier Blocks for Finite Classical Groups at Linear Primes, J. Algebra 432 (2015), 91-128.

[52] Livesey, M., A note on perfect isometries between finite general linear and unitary groups at unitary primes, arXiv:1411.6862. 
[53] Lusztig, G., Irreducible representations of finite classical groups, Invent. Math. 43 (1977), 125175 .

[54] Lusztig, G., Characters of reductive groups over a finite field. Annals of Mathematics Studies, 107. Princeton University Press, Princeton, NJ, 1984.

[55] Lusztig, G., Rationality properties of unipotent representations, J. Algebra 258 (2002), 1-22.

[56] Lusztig, G., Srinivasan, B., The characters of the finite unitary groups, J. Algebra 49 (1977), 167-171.

[57] Macdonald, I.G., Symmetric Functions and Hall Polynomials, 2nd edition, Oxford Mathematical Monographs, Oxford Science Publications, The Clarendon Press, Oxford University Press, New York, 1995.

[58] Mathas, A., The representation theory of the Ariki-Koike and cyclotomic q-Schur algebras. Representation theory of algebraic groups and quantum groups, 261-320, Adv. Stud. Pure Math., 40, Math. Soc. Japan, Tokyo, 2004.

[59] Rouquier, R., 2-Kac-Moody algebras, arXiv:0812.5023v1.

[60] Rouquier, R., Shan, P., Varagnolo, M., Vasserot, E., Categorifications and cyclotomic rational double affine Hecke algebras, Invent. Math. (to appear), arXiv:1305.4456.

[61] Shan, P., Crystals of Fock spaces and cyclotomic rational double affine Hecke algebras, Ann. Sci. Éc. Norm. Supér. (4) 44 (2011), 147-182.

[62] Shan, P., Vasserot, E., Heisenberg algebras and rational double affine Hecke algebras, J. Amer. Math. Soc. 25 (2012), 959-1031.

[63] Srinivasan, B., On CRDAHA and finite general linear and unitary group, arXiv:1411.1714v1.

[64] Takeuchi, M., The group ring of $\mathrm{GL}_{n}(q)$ and the $q$-Schur algebra, J. Math. Soc. Japan 48 (1996), 259-274.

[65] Uglov, D., Canonical bases of higher-level q-deformed Fock spaces and Kazhdan-Lusztig polynomials, in Physical combinatorics (Kyoto 1999), 249-299, Birkhäuser, Boston, 2000.

[66] Yvonne, X., Bases canoniques d'espaces de Fock de niveau supérieur, PhD thesis, University of Caen (2005). 